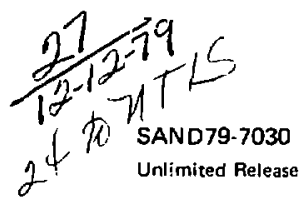

\title{
Creep Behavior of Bedded Salt From Southeastern New Mexico at Elevated Temperature
}

Francis D. Hansen, Kirby D. Mellegard

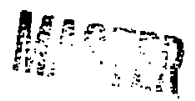

Sandia Laboratories 
SAVU79-7050

Unlinited Release

Printed vovember 1979
D) 1 itribltion

Category LCC 70

TFCHNICAL MEMORANDUM REPOKT RST-0062

\section{CREEP BEUAVTOR OF BEDDED SALT FROM SOUTHEASTERN}

NEW MEXICO AT ELEVATED TEMPERATURE

Submitted To

Sandia Laboratories

Albuguerque, New Nexico

Operated By

Sandia Corporation

for the

Energy Research and Development Adninistration

$B y$

Francis D. Hansen and

Kirby D. Mellegard

November 9, 1977

\author{
of \\ RE/SPEC InC. \\ P. 0. Box 725 \\ Rapid City, South Dakota
}

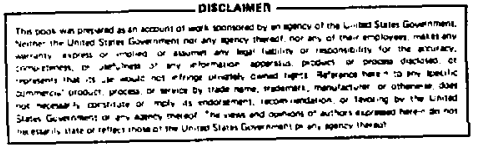

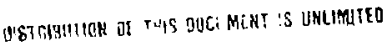




\section{FOREWORD}

This report summarizes the results obtained f-om a series of triaxial creep experiments on specimens of bedied salt recovered from a depth interval of 2605 to 2679 feet in ERDA Hole No. 9 iocated in southeastern New Mexico. The objective of this experimental effort was to determine the time-dependent deformational characteristics of the salt under cifferential stress and elevated temperature. The tests encompassed differential axial stress levels ranging from 1500 to 6000 psi, confining stress levels from zero to 3000 psi, and temneratures of 24,70 and $100^{\circ} \mathrm{C}$. Time durations were variable and ranged from 15 minutes to 20 days. Data analysis consisted of thorough documentation and presentaticn of experimental results and power law fits to the creen straln-time measurelients.

Thi- report was prepared by RE/SPEC Inc. for Sandia Laboratories under Sandia Criporation contract Document No5, 02-885A and 05-7466 under Contract AT (29-1)-789 with the United States Eneron Research ant Development Adminis'ration. The authors are indebted to staff members of RE/SPEC InC. fOI contributions and support during the course of testinx, data analysis and report preparation. Mr. Leslie $A$. Wamer and Mr. Daniel $M$. Schiermeister were instrumental to the entire testing erfort including sample preparation, continual maintenance and initial data acquisition. Dr. Paul F. Fnirk reviewed the technical contents of this report and lent constructive criticism. A special acknowlednement, is in order for Ms. Julie S. Annicchiarico and Ms. Elizabeth A. Speer for their extra efforts in preparation of this report. The authors also would like to thank $D r$. Wolfgang $R$. Wawersik for his support in this effort thronghout the year. 


\section{TABLE OF CONTENTS}

1. INTRODUCTION

2. BACKGROUND AND FROCEDURES

2.1. Test Matrix

2.2. Specimen Preparation

2. 3. Apparatus and Procedure

2.4. Calibration of Testing Apparatus

2.4.1. Calibration of Axiai and Lateral Systems

2.4.2. Calibration of Temperatures

3. DIFFERENTIAL STRESS APPLICATION TO CREEP INITIATION

4. RESULTS OF TRIAXIAL CREEP EXPERIMENTS

4. 1. Introductory Remarks

4.2. Axial strain as a Function of Time

4. 3. Lateral Strain as a Function of Time

$\therefore$

4. 4. Deformeả specimens

$\ddot{i=}$

4.5. Activation Energy

$\therefore:$

4.6. Comparison with Previous Results

$\because 7$

5. CONCLUDTNG REMRRKS

19

LIST OF REFERENCES

APPENDIX A: PLOTS OF DIFFERENTIAL STRESS APPLICATION TO CREEP STAGE 


\section{LIST OF FIGURES}

\section{FIGURE NO.}

Photograph of Testing Apparatus Housing Machine 1 on Right, Machine 2 on Left.

Photograph of Testing Machine 3.

Temperature Calibration Assembly.

Differential Axial Stress as a Function of Azial strain for Stress Application to Initiate Creep Tests at Various Temperatures.

Principal strain Ratio Variations During Differential Stress Application to Initiate Creep Tests at Various Temperatures and Confining Pressures.

Typical Results of Curve Fitting to Experimental Data.

Creep Test Results; Salt from ERDA 9 at 2700 Foot Depth Tested at Room Temperature.

Creep Test Regults; Salt from ERDA 9 at 2700 Foot Depth Tested at $100^{\circ} \mathrm{C}$. 


\section{LIST OF TABLES}

TABLE NO.

PAGE

1

Stramman of Projected RE/SPEC Creen Tests FY77

$\therefore$

Four-Inch Cores from ERDA $g$ and Finished Two-Inch

23 Specimens

3

Sumary of Test Data for Triaxial Compression to

$2:$

Initiate Creep Tests on ERDA 9 salt

4

Sumary of Experimental Axial Strain Data

is

Axial Creep strain at Various Times

6

Lateral Creep Strain at Various Times

$\because \hat{i}$

7

Steafy state Axial Creep

8

Comparison of Experimental Data for Tests Included in

This Report and for Tests as Reported in Reference I 


\section{$R_{S}$ RE/SPEC INC.}

P. O. BOX 725 - RAPID CIIY, 5.D.57709 •605/343.786B

November 9, 1977

\section{TECHNICAL MEHORANDUM REPORT RST-0O62}

TO: Sandia Iaboratories

Albuquerque, NM 87115

Attn.: Dr. Les R. Hill

Dr. Darrell E. Hunson

Dr. Wolfoang $R$. Wawersik

FROH: Mr. Francis D. Hansen

and

Mr. Kirby D. Meliegard

RE/SPEC InC.

P. O. Box 725

Rapid City, SD 57701

SUBJECT: Triaxial Creep Behavior of Bedded salt from southeastern New Mexico at Elevated Temperature (Sandia Cormotion, Contract Nos. 02-8858 and 05-7466).

\section{INTRODUCTION}

This report presents the results of a series of triaxial creep experiments conducted on bedded salt specimens from ERnA Hole 9 in southeastern New Mexico. The salt core and matrix of test conditions were provided by $D r$. Wolfgang $R$. Wawersik, Sandia Laboratories. The purpose of the experiments was to measure creep response of salt at temperatures of 24,70 and $100^{\circ} \mathrm{C}$ under confinement pressures of $n$, 1500, 2000, 2500 and 3000 psi and differential axial stress levels of $1500,3000,4500$ and $6000 \mathrm{psi}$. Test durations ranged from 15 minutes to over 500 hours.

The specimens, obtained by recoring four-inch diameter cores in the axial direction, were nominally two inches in diameter and four inches in length. The crystal size ranged from very small to one-half inch diameter; the specimens contained various amounts of clay impurities. A total of 19 specimens were prepared of which 14 were tested. 
The collected data included axial and lateral strain, axial and confinement stresses, time and temperature. periodically, axial stress was adjusted to account for specimen strain in order to maintain a constant differential stress. Frequency of the stress correction was dependent on the rate of deformation; two or more corrections in a 24 hour period were typical. Data were automatically recorded with a printer, manualiy recoded from the print-out to punched cards and reduced by means of a computer. A preposderance of the data (see section 4.1) was collected in the transient creep regime. In some tests specimen rupture occurred, while in others an accelerating creep rate brought the specimen in contact witi the pressure vessel wall. Also, a considerable amount of data was collected during stress application to creep stress level.

It is the purpose of this report to present all of the data obtained in a concise manner such that use can be made of these results by $D r$. Wawersik in his more comprehensive experimental program. For that matter, the contents have been divided into sections which present summaries of the collective results. The experimental data have been fit with equations which describe transient creep. All of the data is presented in plots in the Appendices. Considerable attention was also given to specimen characterization and measurements after deformation. 


\section{BACKGROUND AND PROCEDURES}

\subsection{Test Matrix}

The Statement of Work Section of Contract 05-7466 specifled elahteen creep (constant stress) tests be conducted on fourteen salt specimens. specifics of principal stress difference, confining pressure, temperature, and duration for the tests were transmittec in a letter from Wolfgang $R$. Wawersik to Paul F. Gnirk on October 22, 1976. Table 1 lists the parameters as projected at that time.

\subsection{Specimen Preparation}

Several four-inch diameter cores of varlous lengths from the ERDA 9 drillhole located in southeastern New Mexico were received by RE/SPEC on April 8, 1977. The four-inch cores were sawed using a bandsaw into five-inch lengths and subsequently recored to two-inch diameter specimens on a vertical milling machine. A thin-walled diamond bit and recirculating saturated brine were used for coring. Table 2 contains a sumary of the cores received and the resultant testable specinens prepared from each piece. Lapping of the specimen ends was accomplished on the vertical milling machine using a diamond wheel. Specimen preparation was extremely precise; ends were parallel \pm 0.001 inches ond diameters were $1.997 \pm 0.002$ inches.

\subsection{Apparatus and Procedure}

All of the tests with the exception of those conducted at room temperature and atmospheric (zero psi) confining pressures (Tests 1, 4, and 9) were conducted on two machines designed bu Dr. Hawersik and shown in Figure 1. Procedural. detail and data acquisition involved with the operation of these machines has been presented previously (1). Tests 1 , 4 and 9 were conducted on a triaxial maciline equipped with precharcer accumulators for maintaining constant axial stross, as shown in Fioure 2 .

Each specimen was first subjected to a hydrostatic stress state and llowed to achieve equilibriun; tests were normally allowed to stabilize overnight. After equllibiation under hydrostatic conditions, differential axial stress was applied at a rate of $100 \mathrm{psi} / \mathrm{min}$. for a majority of the tests. Variances in the rate on five tests ranged from 75 to 275 pal/min. (Table 3). During differential scress application, axial 
displacement data were collected dt one minute intervals on all of the tests; lateral strain data were collected on only those specimens subjected to confinement pressures.

TABLE 1

STRAWMAN OF PROJECTED RE/SPEC CREEP TESTS FYT7

\begin{tabular}{|c|c|c|c|c|}
\hline $\begin{array}{c}\text { TEST } \\
\text { SPECIMENS }\end{array}$ & $\begin{array}{c}\text { TEMPERATURE } \\
\left({ }^{\circ} \mathrm{C}\right)\end{array}$ & $\begin{array}{l}\text { PRINCIPAL STRESS } \\
\text { DIFFERENCE (PSI) }\end{array}$ & $\begin{array}{c}\text { CONEINING } \\
\text { PRESSURE (PSI) }\end{array}$ & $\begin{array}{c}\text { TEST DURATION } \\
\text { (DAYS) }\end{array}$ \\
\hline 1 & 24 & 1500 & 0 & 15 \\
\hline 2 & 70 & 1500 & 0 & 25 \\
\hline 3 & 100 & 1500 & 0 & 10 \\
\hline 4 & 24 & 3000 & 0 & $\leq 10$ \\
\hline 5 & 24 & 3000 & 2500 & 15 \\
\hline 6 & 70 & 3000 & 2500 & 10 \\
\hline 7 & 100 & 3000 & 2500 & 10 \\
\hline 8 & 100 & 1500 & 3000 & 15 \\
\hline 9 & 24 & $1500 / 3000$ & 0 & $7 / 5=12$ \\
\hline 10 & 24 & $1500 / 3000$ & 2500 & $7 / 7=14$ \\
\hline 11 & 100 & $1500 / 3000$ & 0 & $5 / 5=10$ \\
\hline 12 & 100 & $1500 / 3000$ & 2500 & $5 / 5=10$ \\
\hline 13 & 24 & 4500 & 2000 & 10 \\
\hline 14 & 24 & 6000 & 1500 & 10 \\
\hline
\end{tabular}

NOTE: Tests 9, 10, 11, 12 are two stage tests; differenital axial stresses and durations are separated by a slash (/). 
TABLE 2

FOUR-INCH CORES FROM ERDA 9 AND FINISHED TWO-INCH SPECIMENS

\begin{tabular}{|c|c|c|c|}
\hline $\begin{array}{l}\text { ERDA } \\
\text { CORE }\end{array}$ & DEPTH INTERVAL (FT.j & $\begin{array}{c}\text { APPROX. } \\
\text { LENGTH } \\
\text { (IN.) }\end{array}$ & $\begin{array}{c}\text { TWO-TNCH SPECIMENS RECORED AND FINISHED } \\
\text { (TEST - DEPTH (FT.))* }\end{array}$ \\
\hline 1 & 2605.0 to 2606.5 & 18 & 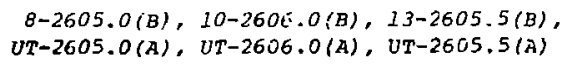 \\
\hline 2 & 2678.0 to 2679.0 & 15 & $\begin{array}{l}12-2678.3(B), 14-2678.7(B), \quad 7-2679.0(B), \\
\text { UT-2678.3(A), } 1 T-2678.7(A), 11-2679.0(A)\end{array}$ \\
\hline 3 & 2678.0 & 5 & $4-2678 . O(A), 9-2678.0(B)$ \\
\hline 4 & 2674.5 & 5 & $6-2674.5(B), 5-2674.5(A)$ \\
\hline 5 & 2622.0 & 5 & $3-2622.0$ \\
\hline 6 & 2668.5 & 5 & $1-2668.5(A), 2-2668.5(B)$ \\
\hline
\end{tabular}

* The prefix UT denotes untested specimen. The suffix (A) or (B) differentiates between specimens recored from the same depth. 


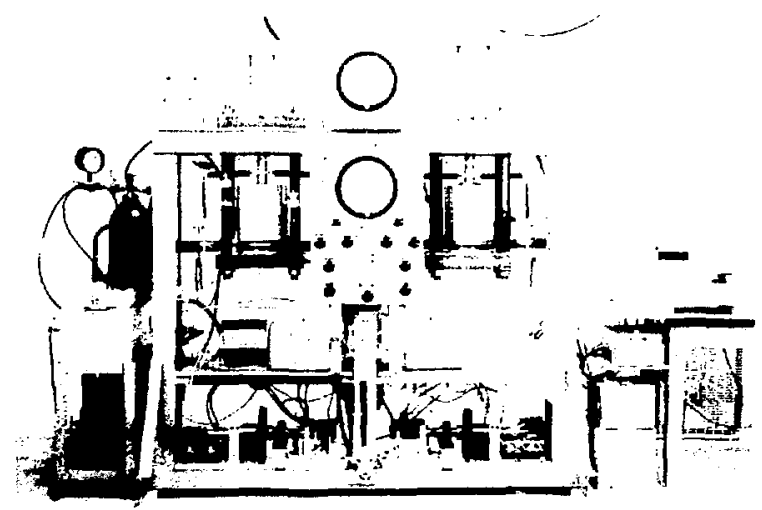

Figure 1. Photograph of Testing Apparatus Housing Machine 1 on Right, Machine 2 on Left.

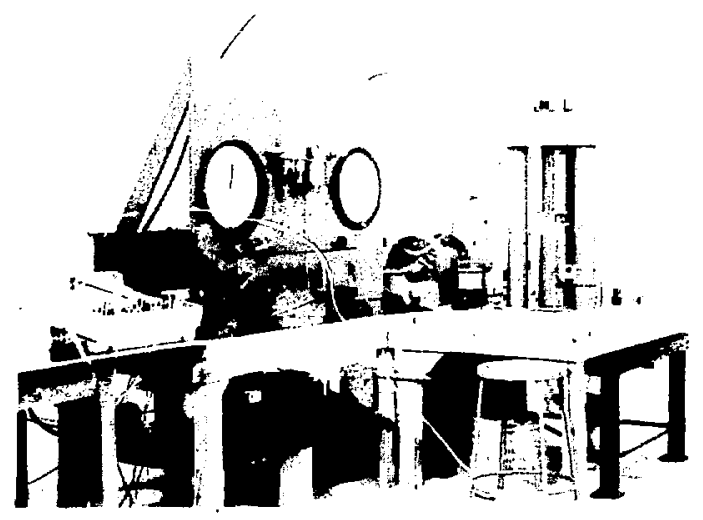

Figure 2. Photograph of Tesing Machine 3. 
Subsequent to achieving the differential axial stress level desired for creep, the axiaj system was closed. A constant axial stress was held on the specimen by means of a one gallon capacity nitrogen charged bladder accumulator. A correction of the difierential axial stress to account for specimen distortion was periodically applied. The recessity and frequency of correctional measures were dependent upon the rate of deformation of the specimen. The correction for cross-sectional area change was taken as:

$$
A_{R}=A_{0}\left(1+\varepsilon_{2}\right)^{2}
$$

where:

$$
\begin{aligned}
& A_{R}=\text { Corrected area of the specimen (sq. in.) } \\
& A_{0}=\text { Original area of the specimen (sq. in.) } \\
& \varepsilon_{2}=\text { Lateral strain. }
\end{aligned}
$$

Where lateral strain measurements were not available, the correction was taken as:

$$
A_{R} \cong A_{0}\left(1+E_{1}\right)
$$

where:

$$
\varepsilon_{1}=2 \varepsilon_{2} \text { (assuming }-\varepsilon_{2} / \varepsilon_{1} \equiv 0.5 \text { ) }
$$

It is recognized that Equation 1 and Equation 2 are not entirely consistent; Equation 2 neglects higher order terms.

\subsection{Callbration of Testing Apparatus}

Calibration of the testing apparatus involved two considerations; $i . e .$, axial and lateral displacements and temperature of the test specimen versus oll temperature. Unlaxial and triaxlal compression tests were performed on metal specimens of brass, aluminum and steel. Calibrations were performed at 24 and $100^{\circ} \mathrm{C}$ temperatures and under confinement pressures of $0,500,1500$ and 3000 psi. A total of 81 separate quasi-statio calibration tests were completed among the three machines. In addition, thermal calibration tests involved assembly of a triaxial experiment with thermocouples placod within a specimen of salt. 


\subsubsection{Calibration of fixial and Lateral Systems}

Uncorfined compression calibrations of metal specimens resulted in initially nonlinear displacement (DCDT) as a function of stress; direct strain gauge data, however, were linear and consistent. Triaxial compression calibrations resulted in linear stress-strain responses with the contribution of the assembly pistons consistent between metal types. Axial calibration consisted of a simple comparison of indirect displacement measurements with strain gauge results. The "machine softness" calculations resulted in axial displacement calibrations essentially identical as obtained and reported previously (1).

calibration of the lateral displacement systen was directed toward determining an effective length of the test colum inside the pressure vessels, $L_{p^{*}}$ Lateral strain measurements obtainer from volumetric displacements were used to calculate $L_{p^{*}}$. Despite the relatively large number of calibration tests, refinement of a value for $L_{p}$ was extremel!" difficult due to scatter in the data. As an illustration, the values for $L_{p}$ ranged from 7.0 to 9.0 inches and were consistently of the order of the values reported previously (1). Influences of $L_{p}$ values on determination of lateral strain are basically a function of the magnitude of strain. That is, for small strains, such as obtained from calibration tests on metals, the value of $L_{p}$ is more significant than it is for larger strains, such as obtained during triaxial creep experiments on rock salt. This partially accounts for thi scatter encountered during calibrations. It was determined that errors in lateral strain calculations due to $L_{n}$ for reduction of actual creep data were minimal (< 1.0\%). For reduction of experimental data in this report, $L_{p}$ values as reported previously (1) were used.

\subsubsection{Calibration of Temperatures}

Temperature of the tests was measured by means of a thermocouple probe inserted in the pressure vessel wall to point flush with the inside diameter (Figure 3). This thermocouple also served as a feedback for temperature control. Calibrations were performed to evaluate the difference between the temperature at the pressure vessel wall and the temperature within the salt specimen at the top, middle and botiom. To accomplish the calibration, three thin(0.020 in. diameter) flexible thermocoupies were inserted into a 1/16" diameter hole drilled in a specimen of Jefferscn Island domal sait ond arranged as illustratod in Figure 3. Tho vessel ofl was heated from 
room temperature to $70^{\circ} \mathrm{C}$ and later to $109^{\circ} \mathrm{C}$ while measurements of temperature were taken from the four themocouples. Tests re conducted with no confinement pressure and with 500 psi confinement pressure.

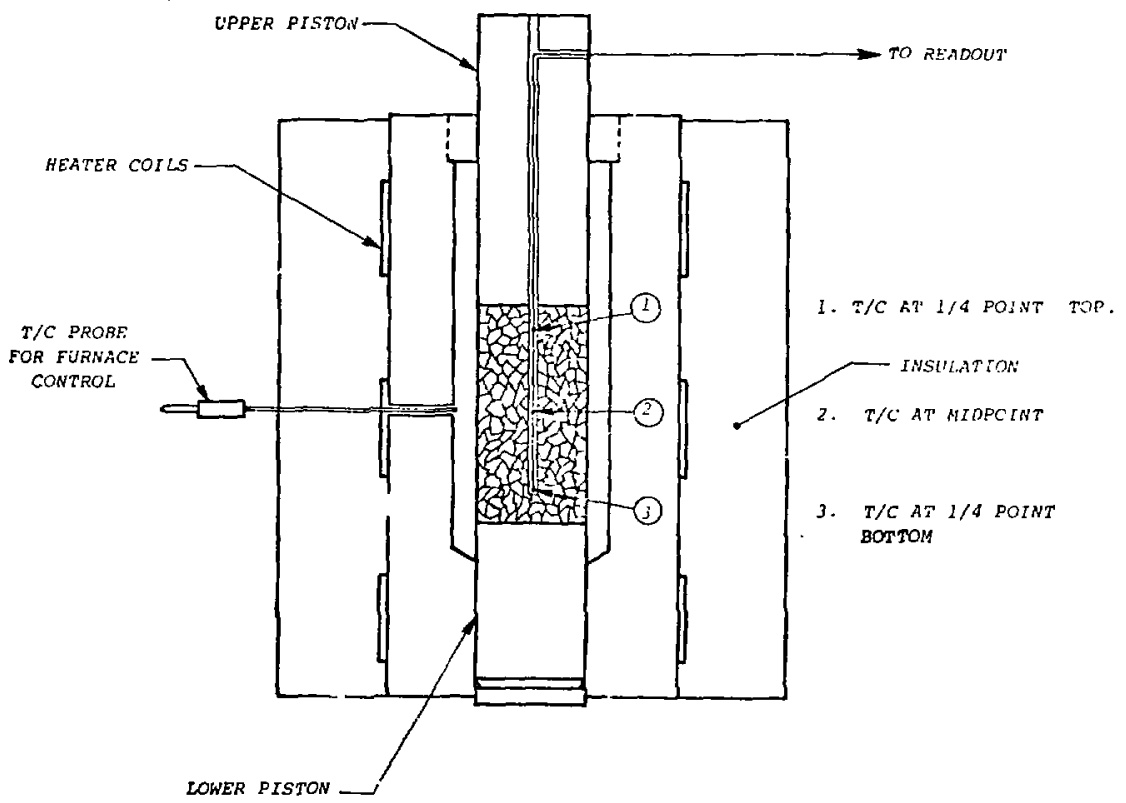

Figure 3. Temperature Calibration Assembly.

As a result of these calibration tests, the internal spectmen temperature was found to be less than the vessel oil temperature by 6 to $8^{\circ} \mathrm{C}$, it $100^{\circ} \mathrm{C}$ and 3 to $5^{\circ} \mathrm{C}$ at $70^{\circ} \mathrm{C}$. As a consequence of this deterninaticn, tests specified $100^{\circ} \mathrm{C}$ were conducted at $106^{\circ} \mathrm{C}$ and tests specified $70^{\circ} \mathrm{C}$ were conducted at $74^{\circ} \mathrm{C}$. Callbration also showed an axial thormal graiient of about $2^{\circ} \mathrm{C}$ to exist in speclmens subjected to elevated temperatures. 


\section{DIFFERENTIAL STRESS APPLTEAITON TO CREEP INITIATION}

After the test assembly had achieved equilibrium under hydrostatic stress conditions, initiation of creep tests involved application of differential stress to the desired level for creep, whereupon the differential stress was held constant. During differential stress application, measurements of axial and, when possible, lateral displacements were made. As these data are representative of limited quasi-static compression tests, the resulting mechanical properties and behavior of the material are of interest. For that matter, Figure 4 illustrates the stress-strain response of all of the tests and Figure 5 illustrates the variation of principal strain ratio of the tests rhen lateral strains were measured. Table 3 is 2 summary of the axial and lateral pressures, temperatures, and load rates, as well as magnitudes of axial and lateral strain. Table 3 also includes an approximation of a modulus of deformation which was calculated by numerical integration of the stress-strain curve over a differential stress range of 0 to 1500 psi. Calculation of the madulus by integration resulted in slightly lower values than secant calculations.

plots of the individual stress-strain curves for stress application are presented in Appendix $A$. 


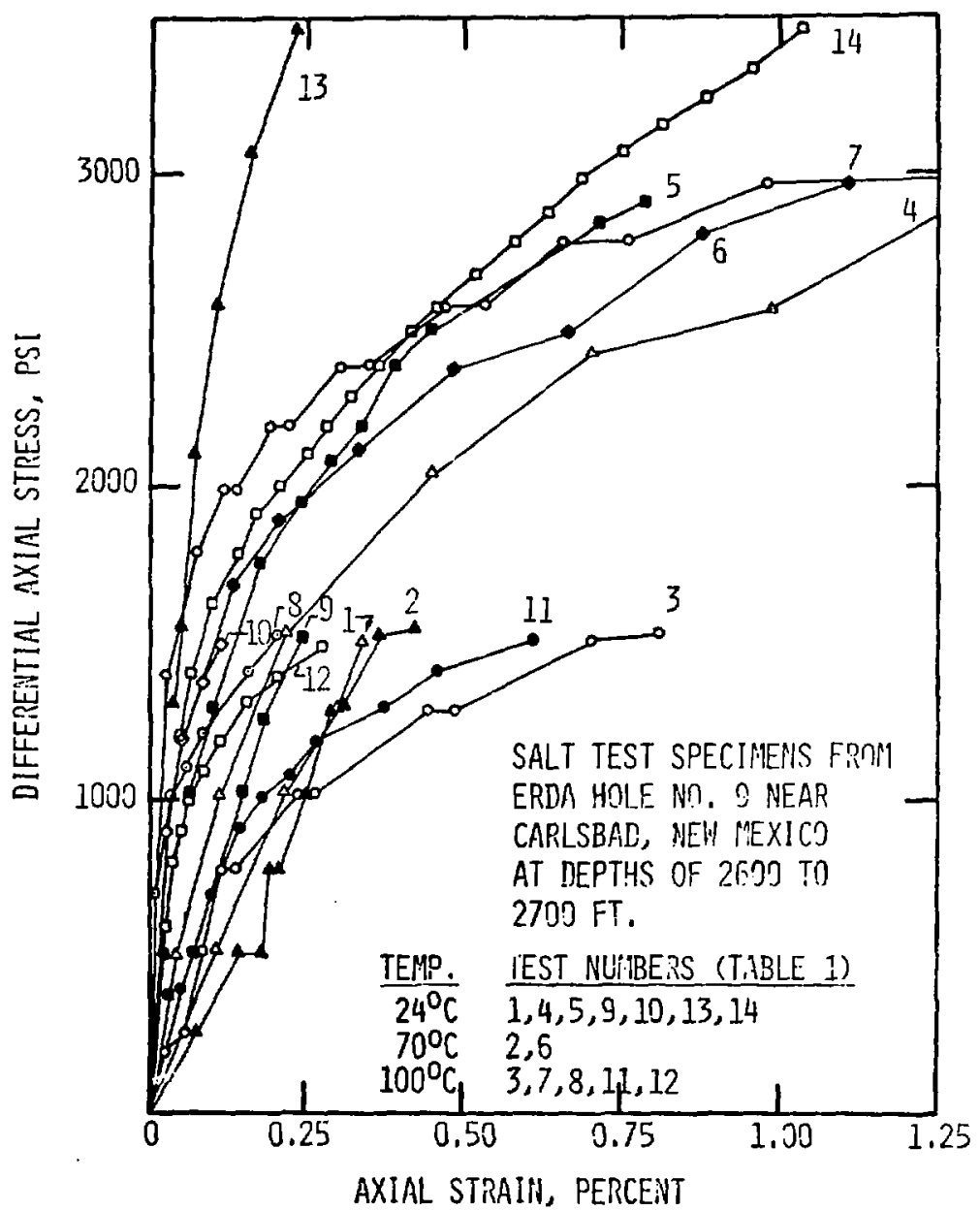

Figure 4. Differential Axial Stpess as a Function of Axial Strain for Stress npplication to Initiate CreEp Tests at Various Temperatures. 


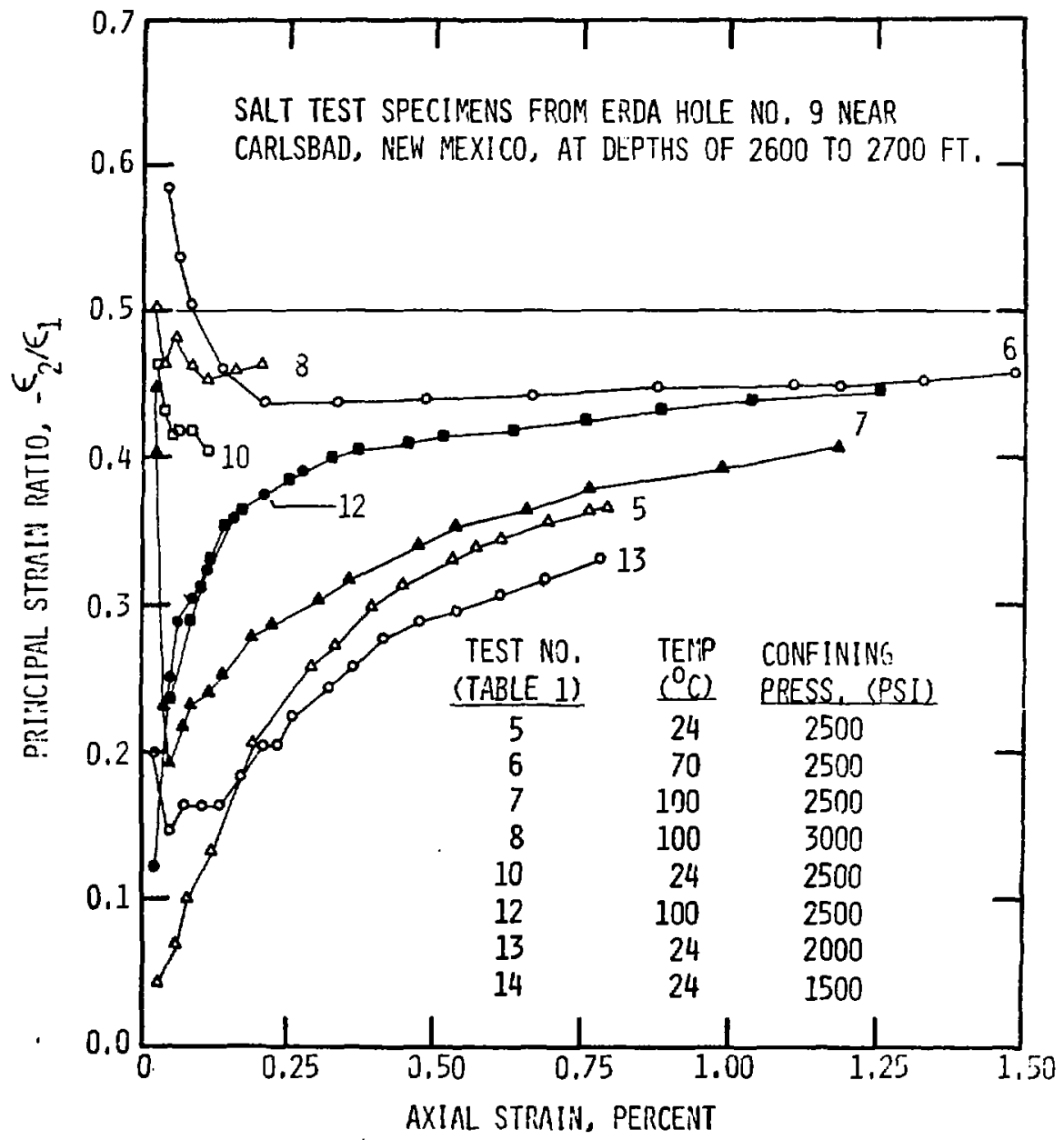

Figure 5. Princ:pal Strain Ratio Variations During Differential Stress Application to Initiate Creep Tests at l'arious Temperatures and Confining Pressures. 
TABLE 3

SUHMARY OF TEST DATA FOR TRTAXIAL COMPRESSION

TO INITIATE CREEP TESTS ON ERDA 9 SALT

\begin{tabular}{|c|c|c|c|c|c|c|c|c|}
\hline $\begin{array}{l}\text { TEst } \\
\text { no. }\end{array}$ & $\begin{array}{c}\text { SAMPLE ORIGIN } \\
\text { (HOLE-DEPTH, FT.) }\end{array}$ & $\begin{array}{l}\text { CONF. } \\
\text { PRESS. } \\
\text { (PSI) }\end{array}$ & $\begin{array}{c}\text { TEST } \\
\text { TENP. } \\
\left(O_{C}\right)\end{array}$ & $\begin{array}{l}\text { LOAD RATE } \\
(P S I / M I N)\end{array}$ & $\begin{array}{l}\text { DIFF. } \\
\text { AXIAL } \\
\text { STRESS } \\
(P S I)\end{array}$ & $\begin{array}{l}\text { TOTAL } \\
\text { AXIAL } \\
\text { STRAIN } \\
(\%)\end{array}$ & $\begin{array}{l}\text { TOTAL } \\
\text { LATERAL } \\
\text { STRAIN } \\
\text { (4) }\end{array}$ & $\begin{array}{c}\text { INTEGRATED } \\
\text { MODULUS FROH } \\
0 \text { to } 1500 \text { PSI } \\
\left(10^{3} \text { PSI }\right)\end{array}$ \\
\hline 1 & $9-2668.5(A)$ & 0 & 24 & $J 25$ & 1500 & 0.34 & -- & 460 \\
\hline 2 & $9-2668.5(B)$ & 0 & 70 & 100 & 1500 & 0.42 & -- & 396 \\
\hline 3 & $9-2622.0$ & 0 & 100 & 100 & 1500 & 0.81 & -- & 275 \\
\hline 4 & $9-2678.0(A)$ & 0 & 24 & 275 & 3000 & 1.45 & -- & 303 \\
\hline 5 & $9-2674.5(A)$ & 2500 & 24 & 75 & 3000 & 79 & 0.29 & 1,431 \\
\hline 6 & $9-2674.5(B)$ & 2500 & 70 & 160 & 3000 & 1.54 & 0.70 & $1,6.98$ \\
\hline 7 & $9-2679.0$ (B) & 2500 & 100 & 100 & 3000 & 1.18 & 0.48 & $2,000 *$ \\
\hline 8 & $9-2605.0(B)$ & 3000 & 100 & 100 & 1500 & 0.20 & 0.09 & 1,204 \\
\hline $9(2)$ & $9-2678.0(8)$ & 0 & 24 & 200 & 1500 & 0.25 & -- & 692 \\
\hline $10(1)$ & $9-2606.0(B)$ & 2500 & 24 & 100 & 1500 & 0.12 & 0.04 & 2,003 \\
\hline $11(1)$ & $9-2679.0(A)$ & 0 & 100 & 100 & 1500 & 0.61 & - & 361 \\
\hline $12(1)$ & $9-2678.3(8)$ & 2500 & 100 & 100 & 1500 & 0.28 & - & 833 \\
\hline 13 & $9-2605.5(8)$ & 2000 & 24 & 100 & 4500 & 0.78 & 0.26 & 3,928 \\
\hline 14 & $9-2678.7$ (B) & 1500 & 24 & 100 & 6000 & 4.37 & 2.11 & 2,418 \\
\hline
\end{tabular}

* Numbers in parentheses indicate stress-strain data if from che initial stage of a multistage cest. Secondary apulicalions ute given in Appendix $A$.

* Approximatcd, DDDT temporsry milfunction. 


\section{RESULTS OF TRIAXIAL CREEP EXPERIMENTS}

\subsection{Introductory Remarks}

This testing effort encompassed 17 creep tests conducted at an ambient temperature of $24^{\circ} \mathrm{C}$ and at elevated temperatures of $70^{\circ} \mathrm{C}$ and $100^{\circ} \mathrm{C}$. Discussion in section 3 covered the behavioral properties of the salt specimens during stress application to the desired stress level for creep. This section will discuss both axial and lateral deformation as a function of time under constant stress conditions. First, the transient creep behavior which comprises the bulk of experimental data will be presented and discussed. Axial and lateral strains have been plotted and fit to power law equations. A section is also devoted to discussion of the specimens at post-failure and photographs of the specimens are presented. Then, brief analyses concerning activation energy and observed steady state creep are presenter. Finally, some comparisons of this set of data to previous data will conclude this section.

Data were collected by means of printed output in engineering units. The $\mathrm{mV}$ signals from transducers were amplified, conditloned and calibrater to englneering units. During intial portions of a test, data were collected at a rate of one set of readings per minute. Dependent on the rate of deformation, the time increments between scans were gradually Increased. Assembly of data into punched cards was accomplished by hand coding. The card decks were processed on a CDC 3400 computer, which reduced displacement measurements to strains, plotted raw data and numerically fit the experimental data with power law functions.

\subsection{Axial Strain as a Function of Tlme}

Intially, lata were plotted in terms of strains as a function of time and given a cursory evaluation. Predominantly, the results displayed transient creep behavior; most strain rates continualiy decreased. This led to a decision to describe the data in terms of $\log _{10}$ strain versus $\log _{10}$ time, which for a majority of the tests resulted in a nearly straight Ine and can be fit by an equation of the form:

$$
E_{2}=k t^{n}
$$

where:

$$
\begin{aligned}
& \varepsilon_{1}=\text { axial straln } \\
& t=\text { time (sec.) (t }>I \text { hour) } \\
& k=\text { experdmontal constant }
\end{aligned}
$$


Evaluation of Equation 3 was by the method of least squares for $t>1$ hour. This procedure is described in more detail in Reference

1. Selection of time greater than one hour for evaluation was awhitraru, but appeared to te a reasonable time for which experimental data showed consistent and 1 inear log-log plots. Equation 3 was then used to describe the complete transient creep response (from $t=0$ ) and for subsequent calculations of strain magnitude and rates. Again, this is a reasonable simplification substantiated by "spot" checking experimental data versus calculated data. For example, actual individual strains were compared to calculated strain magnitudes at a time of cne hour and found to be essentially identical; similarly experimental strain rate data were compared to calculated strain rates and found to be consistentl! of the same order of magnitude. An illustrative example of a typical result is shown in Figure 6-A and 6-B. Figure 6-A illustrates $\log _{10}$ strain as a function of $\log _{10} t 1$ me, including the fit of a least squares line. Figure 6-B is a comparison of the axial strain as a function of time calculated from the resultant equation and the raw experimental data. Experiments which exhibited faster strain rates and greater magnitudes of strain were fit extremely well. Experiments which exhibited slower rates and less strain contained thermal variations and comparison was good but scattered.

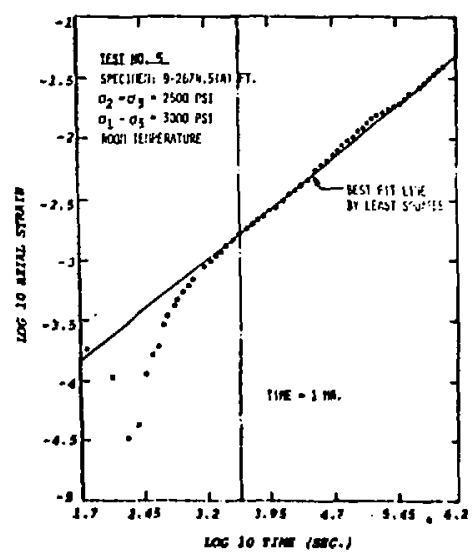

(a)

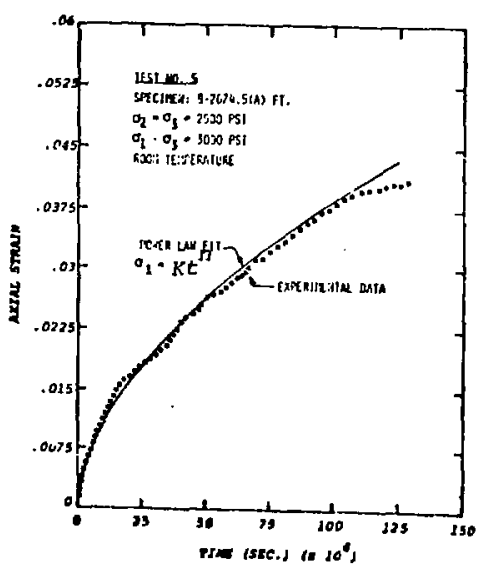

(a)

Figure 6. Typical Rosults of Curve Fitting to Exporimental Data. 
All of the experiments resulted in at least an inltlal portion of creep which was definitely transient; most of the tests conducted at elevated temperature $\left(70^{\circ} \mathrm{C}\right.$ and $\left.100^{\circ} \mathrm{C}\right)$ appeared to exhibit some steadystate creep, thougin Interpretation is somewhat arbitrary. Also, some of the experiments resulted in accelerating creep rate prior to contacting the vessel wall, teminating the test. Tertiary creep iata was omitted for purposes of fitting curves to transient creep. Most of the data appearing steady-state on the time-strain curves also appearec rather linear on $\log _{10}$ time versus $\log _{10}$ strain curves and was included in these calculations. Appendix $B$ contains plots of axial strain as a furction of time as well as $\log _{10}$ axial strain as a functior of $\log _{10}$ time for each of the experiments.

rable 4 contains a summary of the creep data including test conditions, duration and resultant magnitudes of strain. A power law of the form of Equation 3 is listed for the transient portion of each test. Experimental variations, such as cyclic response to ambient temperature variations, make handling of raw experimental data cumbersome. A power law fit facilitates calculation of creep rates and strain magnitudes without the experimenta? scatter. It should be noted that the powers on time and the constant coefficients are jiven with more significant digits than justifiable. The idea is to allow subsequent rounding of the numbers a discretion of the reader.

By use of a computerized multiple regression analysis utlizing raw experimental data, a power law was evaluated which incurporates the influence of temperature and stress as well as time. such a power law may be written:

$$
\varepsilon_{1}=-K t^{n} \sigma^{m} p
$$

where:

$$
\begin{aligned}
& \sigma=\text { differential axial stress (Dsi) } \\
& T=\text { absolute temperature }(K)
\end{aligned}
$$

The other parameters have been previously defined.

As a result of the fourth order multiple regression on experimental data; a power law of the form of Equation 4 is: 
TABLE \&

SUNARY OF EXPERTMENTAL AXIAL STRATN DATA

\begin{tabular}{|c|c|c|c|c|c|c|c|c|c|c|c|}
\hline $\begin{array}{l}\text { TEST } \\
\text { \$OS. }\end{array}$ & SPECIMEN ORIGIN & $\begin{array}{l}\text { TEST } \\
\text { MACH. } \\
\text { NO. }\end{array}$ & $\begin{array}{l}\text { TEST } \\
\text { TENP. } \\
\text { (C) } \\
\end{array}$ & $\begin{array}{c}\sigma_{2} \\
(P S I)\end{array}$ & $\begin{array}{c}\Delta \sigma \\
(P S I)\end{array}$ & $\begin{array}{c}\text { STAGE } \\
\text { OF } \\
\text { TEST }\end{array}$ & DURATION & $\varepsilon_{1}^{*}$ & $E_{2}$ & $\begin{array}{c}\text { LEAST SOUARES FIT } \\
\text { TO EXPERINENTAL DATA } \\
\text { IN TRANSTENT REGIME } \\
\end{array}$ & CONGENTS \\
\hline 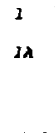 & $s-2663.5(A)$ & 3 & 24 & 0 & 1,500 & $\begin{array}{l}\text { App. } \\
\text { Creop } \\
\text { Rpp. } \\
\text { Creep }\end{array}$ & $\begin{array}{l}12 \mathrm{MTN} . \\
123 \mathrm{HRS} . \\
13 \mathrm{NIN.} \\
361 \mathrm{HRS} .\end{array}$ & $\begin{array}{l}0.34 \\
0.47 \\
0.17 \\
0.07\end{array}$ & $\begin{array}{l}- \\
- \\
-\end{array}$ & $\varepsilon_{1}=3.622\left(10^{-5}\right) t^{0.3631}$ & $\begin{array}{l}\text { Leak Mecessi- } \\
\text { tated, Unloading } \\
\text { at } 123 \text { Hrs., } \\
\text { Test was } \\
\text { Restarted, Data } \\
\text { was Added. }\end{array}$ \\
\hline 2 & $9-2668.5(B)$ & 2 & 70 & 0 & 1,500 & $\begin{array}{l}\text { App. } \\
\text { Creop }\end{array}$ & $\begin{array}{l}15 \mathrm{MIN} . \\
\text { SOI HRS. }\end{array}$ & $\begin{array}{l}0.42 \\
3.27\end{array}$ & 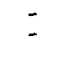 & $E_{1}=2.190 / 10^{-5}, t^{0.4991}$ & Good Test. \\
\hline 3 & $9-2622.0$ & 1 & 100 & 0 & 1,500 & $\begin{array}{l}\text { App. } \\
\text { Creep }\end{array}$ & $\begin{array}{l}15 \text { MTN. } \\
503 \text { HRS. }\end{array}$ & $\begin{array}{r}0.81 \\
10.87\end{array}$ & $\overline{-}$ & $c_{1}=1.800\left(10^{-4}\right) 0^{0.4400}$ & Good Test. \\
\hline$\checkmark$ & $9-2678.0(A)$ & 3 & 24 & 0 & 3,000 & $\begin{array}{l}\text { App. } \\
\text { Greep }\end{array}$ & $\begin{array}{l}11 \text { NIN. } \\
3 \text { HRS. }\end{array}$ & $\begin{array}{l}1.45 \\
2.75\end{array}$ & $\overline{-}$ & $\varepsilon_{1}=5.475\left(10^{-5}\right)=0.6151$ & $\begin{array}{l}\text { Spacimen } \\
\text { Rupcured. }\end{array}$ \\
\hline 5 & $9-2674.5(A)$ & 2 & 24 & 2,500 & 3,000 & $\begin{array}{l}\text { App. } \\
\text { Creep }\end{array}$ & $\begin{array}{l}40 \mathrm{HIN} \text {. } \\
360 \mathrm{HRS} \text {. }\end{array}$ & $\begin{array}{l}0.79 \\
4.11\end{array}$ & $\begin{array}{l}0.29 \\
1.80\end{array}$ & $\varepsilon_{1}=1.756\left(10^{-5}\right) t^{0.5572}$ & Good Test. \\
\hline 6 & $9-267.5(B)$ & 1 & 70 & 2,500 & 3,000 & $\begin{array}{l}\text { App. } \\
\text { Creep }\end{array}$ & $\begin{array}{l}19 \text { MIN. } \\
160 \text { HRS. }\end{array}$ & $\begin{array}{r}1.54 \\
43.00\end{array}$ & $\begin{array}{r}0.70 \\
20.50\end{array}$ & $\varepsilon_{l}=6.151\left(10^{-5}, t^{0.61,8}\right.$ & $\begin{array}{l}\text { Accelerated } 6 \\
80 \text { to } 100 \text { Hrs.. } \\
\text { Contacted Vessed } \\
\text { walls a About } \\
150 \text { Hrs. }\end{array}$ \\
\hline 7 & $9-2679.0(8)$ & 1 & 300 & 2,500 & 3,000 & $\begin{array}{l}\text { App. } \\
\text { Creep }\end{array}$ & $\begin{array}{l}31 \text { MIN. } \\
43 \text { HRS. }\end{array}$ & $\begin{array}{r}1.12 \\
39.81\end{array}$ & $\begin{array}{r}0.48 \\
28.36\end{array}$ & $\varepsilon_{I}=1.391\left(10^{-4}\right) t^{0.6230}$ & $\begin{array}{l}\text { Accelerated 20 } \\
\text { lrs., contücted } \\
\text { lessel rydl } \\
\text { End of Test. }\end{array}$ \\
\hline
\end{tabular}

- Scrain indicated in this column is totil strain at test terminjtion. The jenst squares equation fits anly the t: snsient dota. 
TABLE 4 (CONT'D)

SUMMARY OF EXPERIMTNTAL STRAIN DATA

\begin{tabular}{|c|c|c|c|c|c|c|c|c|c|c|c|}
\hline $\begin{array}{l}\text { TEST } \\
\text { No. }\end{array}$ & SPECINEN ORIGIN & $\begin{array}{l}\text { TEST } \\
\text { WhCH. } \\
\text { NO. }\end{array}$ & $\begin{array}{l}\text { TEST } \\
\text { TEMP. } \\
(\mathrm{C})\end{array}$ & $\begin{array}{c}\sigma_{2} \\
(P S I)\end{array}$ & $\begin{array}{c}\Delta \sigma \\
(\boldsymbol{P S} I)\end{array}$ & $\begin{array}{l}\text { STAGE } \\
\text { OF } \\
\text { TEST }\end{array}$ & DURATION & $c_{1}$ & $\begin{array}{l}E_{2} \\
(x)\end{array}$ & $\begin{array}{l}\text { LEAST SQUARES FIT } \\
\text { TO EXPERTMENTAL DATA } \\
\text { IN TRANSIENT REGIME }\end{array}$ & COMHENTS \\
\hline 8 & $9-2605.0(B)$ & 1 & 100 & 3,000 & 1,500 & $\begin{array}{l}\text { App. } \\
\text { Crtep }\end{array}$ & $\begin{array}{l}15 \text { Min. } \\
362 \text { Hrs. }\end{array}$ & $\begin{array}{l}0.20 \\
6.55\end{array}$ & $\begin{array}{l}0.09 \\
3.14\end{array}$ & $\varepsilon_{1}=1.255\left(10^{-5}\right) e^{0.6022}$ & Good Test \\
\hline $9-5 T 51$ & $9-2678.0(8)$ & 3 & 24 & 0 & 1,500 & $\begin{array}{l}\text { App. } \\
\text { Creep }\end{array}$ & $\begin{array}{l}7 \text { Min. } \\
162 \text { Hrs. }\end{array}$ & $\begin{array}{l}0.24 \\
0.36\end{array}$ & $\tilde{-}$ & $\varepsilon_{I}=3.886\left(10^{-5}\right) t^{0.3368}$ & Good Test \\
\hline $9+5 T \sigma 2$ & $9-2678.0(8)$ & 3 & 24 & 0 & 3,000 & $\begin{array}{l}\text { App. } \\
\text { Creep }\end{array}$ & $\begin{array}{l}15 \mathrm{Min} . \\
15 \mathrm{Min} \text {. }\end{array}$ & $\begin{array}{l}0.87 \\
0.89\end{array}$ & $\overline{-}$ & -- & $\begin{array}{l}\text { Sporcimen } \\
\text { Ruptured }\end{array}$ \\
\hline & & 1 & 24 & 2,500 & 1,500 & $\begin{array}{l}\text { App. } \\
\text { Creep }\end{array}$ & $\begin{array}{l}15 \text { Min. } \\
170 \text { Hrs. }\end{array}$ & $\begin{array}{l}0.12 \\
0.35\end{array}$ & $\begin{array}{l}0.05 \\
0.22\end{array}$ & $E_{1}=2.805\left(10^{-5}\right) t^{0.3579}$ & Good Test \\
\hline $10-\operatorname{stg} 2$ & $9-2606.0(B)$ & 1 & 24 & 2,500 & 3,000 & $\begin{array}{l}\text { App. } \\
\text { Creep }\end{array}$ & $\begin{array}{l}15 \mathrm{Min} . \\
189 \mathrm{HrS} .\end{array}$ & $\begin{array}{l}0.51 \\
2.91\end{array}$ & $\begin{array}{l}0.22 \\
1.43\end{array}$ & $\varepsilon_{1}=3.867\left(10^{-5}\right) e^{0.5028}$ & Good Tese \\
\hline $\begin{array}{l}11-\operatorname{STG1} \\
11-\operatorname{stg} 2\end{array}$ & $\begin{array}{l}9-2679.0(A) \\
9-2679.0(A)\end{array}$ & 2 & $\begin{array}{l}100 \\
100\end{array}$ & 0 & $\begin{array}{l}1,500 \\
3.000\end{array}$ & $\begin{array}{l}\text { App. } \\
\text { Crepp } \\
\text { App. }\end{array}$ & $\begin{array}{l}15 \text { Min. } \\
124 \text { His. } \\
21 \text { Min. }\end{array}$ & $\begin{array}{l}0.61 \\
7.82 \\
6.97\end{array}$ & $\begin{array}{l}- \\
-\end{array}$ & $c_{1}=3.978\left(10^{-5}, t^{0.5804}\right.$ & $\begin{array}{l}\text { Stage 1, Good } \\
\text { Test, Contacted } \\
\text { Vessel Hall } \\
\text { Second Stress } \\
\text { Application }\end{array}$ \\
\hline 12-STG1 & $9-2678-3(B)$ & 1 & 100 & 2,500 & 1,500 & $\begin{array}{l}\text { App. } \\
\text { Creep }\end{array}$ & $\begin{array}{l}15 \text { Min. } \\
137 \text { Hrs. }\end{array}$ & $\begin{array}{l}0.28 \\
5.27\end{array}$ & $\begin{array}{l}0.11 \\
2.67\end{array}$ & $\varepsilon_{1}=1.980\left(10^{-5}\right) t^{0.6000}$ & Good Test \\
\hline $12-5 T G 2$ & $9-2678.3(B)$ & 1 & 200 & 2,500 & 3.000 & $\begin{array}{l}\text { App. } \\
\text { creep }\end{array}$ & $\begin{array}{l}15 \text { Min. } \\
13 \text { Hrs. }\end{array}$ & $\begin{array}{c}1.66 \\
20.53\end{array}$ & $\begin{array}{c}0.82 \\
11.18\end{array}$ & $c_{d}=2.883\left(10^{-4}\right) t^{0.6060}$ & $\begin{array}{l}\text { Steady State } \\
\text { for Last } 6 \text { Hrs. }\end{array}$ \\
\hline 13 & $9-2605.5(B)$ & 1 & 24 & 2,000 & 4,500 & $\begin{array}{l}\text { App. } \\
\text { Creep }\end{array}$ & $\begin{array}{l}45 \mathrm{~min} . \\
262 \mathrm{Hrs} .\end{array}$ & $\begin{array}{c}0.78 \\
12.18\end{array}$ & $\begin{array}{l}0.26 \\
6.55\end{array}$ & $c_{1}=1.136\left(10^{-4}\right) t^{0.5090}$ & Good Test \\
\hline 19 & $9-2678.7(B)$ & 1 & 24 & 1,500 & 6,000 & $\begin{array}{l}\text { App. } \\
\text { Creop }\end{array}$ & $\begin{array}{l}60 \text { Min. } \\
\text { B9 } \text { Hrs. }\end{array}$ & $\begin{array}{c}4.37 \\
22.77\end{array}$ & $\begin{array}{l}2.11 \\
13.51\end{array}$ & $\varepsilon_{1}=7.538\left(10^{-4}\right) 0^{0.4457}$ & $\begin{array}{l}\text { Torminsted Just } \\
\text { Prior to } \\
\text { Contacting } \\
\text { Vessel hall }\end{array}$ \\
\hline
\end{tabular}




$$
\varepsilon_{1}=1.1\left(10^{-35}\right) t^{0.4656} \sigma^{2.475} T^{8.969}
$$

rable 5 is a tabulation of the various axial creep strains for 16 experiments to which the data were fit with power law of the form of Equation 3. Near the bottom of Table 5 are several comparative values of axial strains as calculated using the power law given above. Subsequent Figures 7 and 8 are plots of experimental data at differential stress levels of 1500, 3000 and 4500 psi at room temperature and at differential stress levels of 1500 and $3000 \mathrm{psi}$ at $100^{\circ} \mathrm{C}$, respectively. Superimposed on actual date plots are the curves calculated on the basis of the best fit power law, Ecuation 4-a. Notably, the power law fits a majority of the experimental data quite well with the exception of tests at a differential stress level of $3000 \mathrm{psi}$ at $100^{\circ} \mathrm{C}$, Tests 7 and 12 - stage 2 . Both of these experiments exhibited large strain and relatively rapid $\left(10^{-6} / \mathrm{sec}.\right)$ strain rates. For the most part, those experiments exhibiting predominently transient creep are modeled well with the power law equation.

\subsection{Lateral Strain as a Function of Time}

Lateral strain response was measured during ten of the creep experiments. Lateral strain was measured volumetrically by means of a servo-control system which controls lateral pressure. As a consequence, the volumetric changes of experiments with confinement pressures of zero psi (atmospheric) could not be measured. Lateral strain data was acquired by measuring the turns of a potentiometer in intimate contact with the cylinder of the servometer. Lateral data acquired was handled in the same manner as axial strain, first documented, plotted and then fitted to equations by least squares.

Appendix $C$ contains the indlvidual plots of lateral strain as a function of time and $\log _{10}$ lateral strain as a function of $\log _{10}$ time. Collectively. the qualitative shape of the lateral strain curves are the same as the corresponding axial strain curves. Simlarly, the experimental curves lent themselves well to a power law curve flt of lateral strain as a function of time of the form of Eguation 3. To sumarize the results, Table 6 was prepared which lists test information, a power law equation which fits the transient experimental data, and calculations of principal strain ratios. overall, the amount of data generated in these tests is too small to arrive at definitive conclusions. The majority of the results appears to indicate constant volume deformation. Response of lateral strain as a function of 
TABLE 5

MXIAL CREEP STRAIN AT VARIOUS TIMES

\begin{tabular}{|c|c|c|c|c|c|c|c|c|c|}
\hline \multirow{2}{*}{$\begin{array}{l}\text { TEST } \\
\text { mo. }\end{array}$} & \multirow{2}{*}{$\stackrel{\Delta 0}{\Delta}$} & \multirow{2}{*}{$\begin{array}{l}\text { TEसP } \\
\text { ("C) }\end{array}$} & \multirow{2}{*}{$\begin{array}{l}\text { LEAST SOUARES } \\
\text { EQUATYON }\end{array}$} & \multicolumn{6}{|c|}{ AXIAL STRAIN AT VARTOUS TIMES } \\
\hline & & & & 4 HRS. & 12 HRS. & 1 DAY & 2 DAYS & 5 maYs & 10 DAYs \\
\hline 1 & 1,500 & 24 & $\varepsilon_{1}=3.622110^{-5} j t^{0.3631}$ & $1.17\left(10^{-3}\right)$ & $1.75\left(10^{-3}\right)$ & $2.25\left(10^{-3}\right)$ & $2.89\left(10^{-3}\right)$ & $4.04\left(10^{-3}\right)$ & $5.18\left(10^{-3}\right)$ \\
\hline$z$ & 1,500 & 70 & $\varepsilon_{1}=2.190110^{-}$ & $2.62\left(10^{-3}\right)$ & $4.51\left(10^{-3}\right)$ & $6.37\left(10^{-3}\right)$ & $9.01\left(10^{-3}\right)$ & 0.0142 & 0.0201 \\
\hline 3 & 1,500 & 200 & $E_{1}=1.80011$ & 0.0122 & 0.0197 & 0.0268 & 0.0363 & 0.0543 & 0.0737 \\
\hline 4 & 3,000 & 24 & $E_{1}=5.475120^{-5}$ & 0.0198 & $(0.0390)$ & -- & - & -- & -- \\
\hline 5 & 3,000 & 24 & $E_{1}=1.756\left(10^{-5}\right.$ & $3.64\left(10^{-3}\right)$ & $6.72\left(10^{-3}\right)$ & $9.89\left(10^{-3}\right\}$ & 0.0146 & 0.0242 & 0.0357 \\
\hline 6 & 3,000 & 70 & $E_{1}=6.151110^{-5}$ & 0.0219 & 0.0429 & 0.0659 & 0.1009 & 0.1770 & $(0.2693)$ \\
\hline 7 & 3,000 & 100 & $E_{1}=1.391010^{-}$ & 0.0542 & 0.1075 & 0.1655 & $(0.2549)$ & -- & - \\
\hline 8 & 1,500 & 100 & $1.255\left(10^{-}\right.$ & $4.01\left(10^{-3}\right)$ & $7.76\left(10^{-3}\right)$ & 0.0118 & 0.0179 & 0.0311 & 0.0472 \\
\hline 9 & 1,500 & 24 & $=3.886110^{-}$ & $9.7:\left(10^{-4}\right)$ & $1.02\left(10^{-3}\right)$ & $2.79\left(10^{-3}\right)$ & $2.26\left(10^{-3}\right)$ & $3.07\left(10^{-3}\right)$ & $\left(3.88\left(10^{-3}\right)\right)$ \\
\hline $10(1)$ & 1,500 & 24 & $\varepsilon_{1}=2.805\left(10^{-5}\right) t^{0.3579}$ & $8.63\left(10^{-4}\right)$ & $1.28\left(10^{-3}\right)$ & $1.64\left(10^{-3}\right)$ & $2.10\left(10^{-3}\right)$ & $2.92\left(10^{-3}\right)$ & $\left(3.74\left(10^{-3}\right)\right)$ \\
\hline $10(2)$ & 3,000 & 24 & $\varepsilon_{1}=3.467\left(10^{-}\right.$ & $4.26\left(10^{-3}\right)$ & $7.39\left(10^{-3}\right)$ & 0.0105 & 0.0148 & 0.0235 & $(0.0333)$ \\
\hline 11 & 1,500 & 100 & $\varepsilon_{1}=3.978110^{-5}$ & 0.0103 & 0.0195 & 0.02916 & 0.0436 & 0.0742 & $(0.2110)$ \\
\hline $12(1)$ & 1,500 & 100 & $\varepsilon_{1}=1.980\left(10^{-5}\right)$ & $6.19\left(10^{-3}\right)$ & 0.0120 & 0.0181 & 0.0275 & 0.0476 & $(0.0722)$ \\
\hline $12(2)$ & 3,000 & 100 & $=2.883\left(10^{-4}\right) t^{0}$ & 0.0955 & 0.1858 & $(0.2827)$ & -- & -. & -- \\
\hline 13 & 4,500 & 24 & $E_{1}=1.136\left(10^{-4}\right) t^{0.5090}$ & 0.0149 & 0.0260 & 0.0370 & 0.0526 & 0.0839 & 0.1193 \\
\hline 14 & 6,000 & 24 & $E_{2}=7.538\left(20^{-4}\right) t^{0.4457}$ & 0.0538 & 0.0878 & 0.1295 & 0.16 .30 & 10.24492 & $=$ \\
\hline & 1,500 & 24 & $\varepsilon_{1}=1.203\left(10^{-5}\right) t^{0.4656}$ & $1.04\left(10^{-3}\right)$ & $1.73\left(10^{-3}\right)$ & $2.39\left(10^{-3}\right)$ & $3.30\left(10^{-3}\right)$ & $5.06\left(10^{-3}\right)$ & $6.99\left(10^{-3}\right)$ \\
\hline$\frac{2}{5}$ & 3,000 & 24 & $E_{1}=6.690\left(10^{-5}\right.$ & $5.78\left(10^{-3}\right)$ & $9.63\left(10^{-3}\right)$ & 0.0133 & 0.0184 & 0.0281 & 0.0389 \\
\hline 象象 & 4,500 & 24 & $t_{1}=1.820\left(10^{-4}\right) t^{0.4656}$ & 0.0158 & 0.0263 & 0.0363 & 0.0501 & 0.0768 & 0.1060 \\
\hline 5. & 1,500 & 100 & $\varepsilon_{1}=9.290\left(10^{-5}\right) t^{0.4656}$ & $8.02\left(10^{-3}\right)$ & 0.0134 & 0.0185 & 0.0255 & 0.0391 & 0.0539 \\
\hline & 3,000 & 100 & $E_{1}=5.160110^{-}$ & 0.0446 & 0.0793 & 0.2027 & 0.1418 & 0.2172 & 0.2949 \\
\hline
\end{tabular}

- Numbers appearing in parentheses are extrapoluted bcyond experiment duration. 


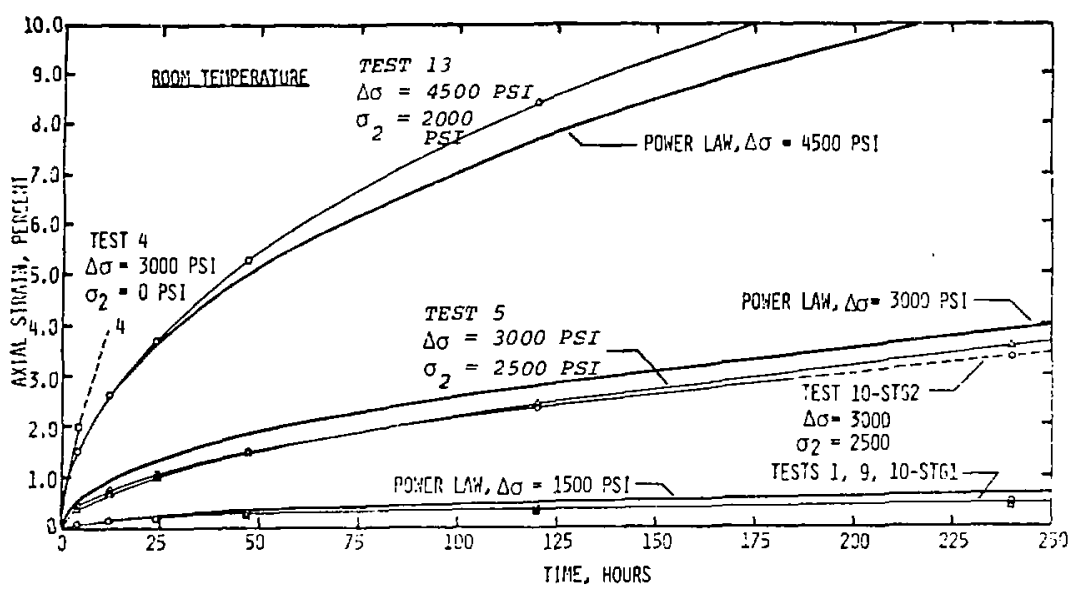

figure 7 . Creep test Results; Salt from erda 9 at 2700 foot Depth tested at room temperature,

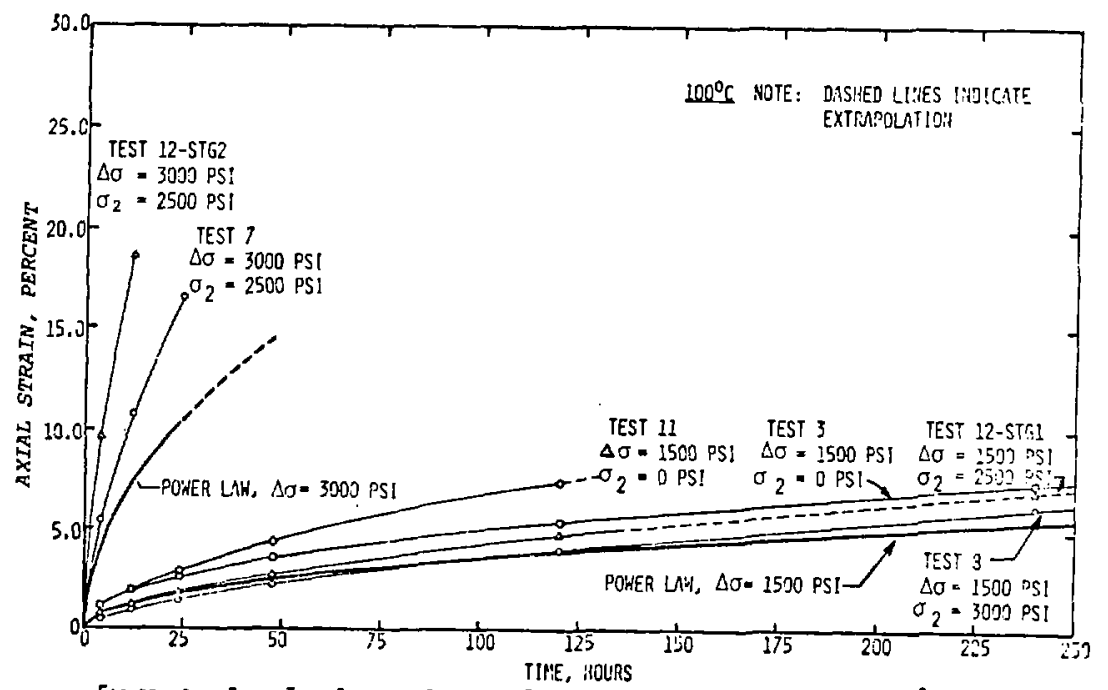

Figure 8 . Creep test results; Salt fad eado 9 at 2700 foot Depth tested at $100^{\circ}$.. 
TABLE 6

IATERAL CREEP STRAIN AT VARJOUS TIMES

\begin{tabular}{|c|c|c|c|c|c|c|c|c|c|}
\hline \multirow{2}{*}{$\begin{array}{l}\text { TEST } \\
\text { NO. }\end{array}$} & \multirow{2}{*}{$\begin{array}{c}\text { AO } \\
\text { (PSI) }\end{array}$} & \multirow{2}{*}{ TEHP. } & \multirow{2}{*}{$\begin{array}{c}\text { LEAST SQUARES } \\
\text { EQUATION }\end{array}$} & \multicolumn{6}{|c|}{ LATERAL STRAIN AT VARIOUS TIHES } \\
\hline & & & & 4 HRS. & 12 HRS. & $1 D A Y$ & 2 DAYS & 5 DAYS & 10 DAYS \\
\hline \multirow[t]{2}{*}{5} & 3,000 & 24 & $\varepsilon_{2}=9.569\left(10^{-6}\right) t^{0.5386}$ & \multirow{2}{*}{$\begin{array}{l}1.66\left(10^{-3}\right) \\
0.46\end{array}$} & \multirow{2}{*}{$\begin{array}{l}3.00\left(10^{-3}\right) \\
0.45\end{array}$} & \multirow{2}{*}{$\begin{array}{l}4.36\left(10^{-3}\right) \\
0.44\end{array}$} & \multirow{2}{*}{$\begin{array}{l}6.34\left(10^{-3}\right) \\
0.43\end{array}$} & \multirow{2}{*}{$\begin{array}{l}0.0104 \\
0.43\end{array}$} & \multirow{2}{*}{$\begin{array}{l}0.015: \\
0.42\end{array}$} \\
\hline & & \multicolumn{2}{|c|}{ PRINCIPAL STRAIN RATIOS } & & & & & & \\
\hline \multirow[t]{2}{*}{$6 *+$} & 3,000 & 70 & $E_{2}=2.710\left(10^{-5}\right) t^{0.6296}$ & \multirow{2}{*}{$\begin{array}{l}0.0102 \\
0.47\end{array}$} & \multirow{2}{*}{$\begin{array}{l}(0.0202) \\
0.47\end{array}$} & \multirow[t]{2}{*}{$\cdots$} & \multirow[t]{2}{*}{--} & \multirow[t]{2}{*}{$\cdots$} & \multirow[t]{2}{*}{-- } \\
\hline & & \multicolumn{2}{|c|}{ PRINCIPAL STRAIN RATIOS } & & & & & & \\
\hline \multirow[t]{2}{*}{7} & 3,000 & 200 & $\varepsilon_{2}=4.751\left(10^{-5}\right) t^{0.6040}$ & \multirow{2}{*}{$\begin{array}{l}0.0274 \\
0.51\end{array}$} & \multirow{2}{*}{$\begin{array}{l}0.0569 \\
0.53\end{array}$} & \multirow{2}{*}{$\begin{array}{l}0.0901 \\
0.58\end{array}$} & \multirow{2}{*}{$\begin{array}{l}(0.1427) \\
0.56\end{array}$} & \multirow[t]{2}{*}{--} & \multirow[t]{2}{*}{$\cdots$} \\
\hline & & \multicolumn{2}{|c|}{ PRINCIPAL STRATN RATTOS } & & & & & & \\
\hline \multirow[t]{2}{*}{$\boldsymbol{B}$} & 1,500 & 100 & $\varepsilon_{2}=8.532\left(10^{-6}\right) t^{0.5721}$ & \multirow{2}{*}{$\begin{array}{l}2.04\left(10^{-3}\right) \\
0.51\end{array}$} & \multirow{2}{*}{$\begin{array}{l}3.83\left(10^{-3}\right) \\
0.49\end{array}$} & \multirow{2}{*}{$\begin{array}{l}5.69\left(10^{-3}\right) \\
0.48\end{array}$} & $8.46\left(10^{-3}\right)$ & 0.0143 & 0.0212 \\
\hline & & PRINCIPA & STRAIN RATIOS & & & & & 0.46 & 0.45 \\
\hline $10-1$ & 3,000 & 28 & $c_{2}=8.312\left(10^{-6}\right) t^{0.4215}$ & $4.70\left(10^{-4}\right)$ & $7.47\left(10^{-4}\right)$ & $1.00\left(10^{-3}\right)$ & $1.34\left(10^{-3}\right)$ & $1.97\left(10^{-3}\right)$ & $\left(2.64\left(10^{-3}\right)\right)$ \\
\hline & & PRTNCSPA & STRAIN RATTOS & & 0.58 & & 0.6 & & 0.71 \\
\hline $10-2$ & 3,000 & 24 & $E_{z=1.246\left(10^{-5}\right) t t^{0.5157}}$ & $1.91\left(20^{-3}\right)$ & $3.41\left(10^{-3}\right)$ & $4.91\left(10^{-3}\right)$ & $7.06\left(10^{-3}\right)$ & 0.0114 & $(0.0165)$ \\
\hline & & PRINCTPA & STRAIN RATIOS & & 0. & & & 0.49 & 0.50 \\
\hline $12-1$ & 1,500 & 100 & $\varepsilon_{2}=9.892\left(10^{-6}\right) t^{0.6006}$ & $3.11\left(10^{-3}\right)$ & $6.02\left(10^{-3}\right)$ & $9.12\left(10^{-3}\right)$ & 10.0138 & 0.0240 & $(0.0364)$ \\
\hline & & PEINCIPS & STFAIN RATIOS & & & & 0.50 & 0.50 & 0.50 \\
\hline $12-2$ & 3,000 & 100 & $\varepsilon_{2}=1.005\left(10^{-4}\right) t^{0.6437}$ & 0.0477 & 0.0965 & $(0.1513)$ & --- & $\cdots$ & -- \\
\hline & & PRINCTPA & STRAIN RATIOS & & & & & & \\
\hline 13 & 4,500 & 24 & $\varepsilon_{2}=3.780\left(10^{-5}\right) t^{0.5427}$ & $6.83\left(10^{-3}\right)$ & 0.0124 & 0.0181 & 0.0263 & 0.0432 & 0.0630 \\
\hline & & PRINCIPA & STRAIN RAYTOS & 0.46 & & 0.49 & 0.50 & 0.51 & 0.53 \\
\hline is & 6,000 & 24 & $c_{2}=2.690\left(10^{-4}\right) \pm^{0.4832}$ & 0.0 & 0.0467 & 0.0653 & 0.0913 & $(0.1422)$ & -- \\
\hline & & PRIMCIPA & STRAIN RATTOS & & & & & & \\
\hline
\end{tabular}

- Numbers dppearing in parentheses are extrapolated beyond ac tual experimental duration.

- Lateral measurements were lost after eight hours dise to broken limit switch. 
time was near one-half the magnitude of the axial strain, and the powers on time, $t$, in the least squares equations are nearly identical between equations for axial and lateral strain.

As noted in section 3 (Figure 5), the principal strain ratio for the varfous tests was generally trending toward 0.5 . During the creep tests, the principal strain ratio slowly increased with time to values greater than 0.5. A notable exception was Test 5, which was contained in Vessel 2. Vessel 2 is prone to very small leaks, which probably negated the increase in principal strain ratio. Another notable exception is Test 10-stage 1. This variation, and consequential high values for principal strain ratio, can probably be attributed to the very small magnitudes measured. Some cyclic thermal drift is inherent to all tests; when very small strains are involved, the drift causes a relatively greater percent error.

\subsection{Deformed specimens}

The purpose of this section is to present photographs of the specimen along with general description of the specimens after testing. Though these specimens were obtained from the same general location, ERDA 9 2600 to 2700 foot depth, considerable variance between specimens exists. Figures 9 through 12 are photographs of the tested as wall as untested spocimens concerned in this experimental program. A general knowledge of tise specimen characterization may assist in qualitative interpretation of data. For that matter, Appendix $D$ contains general descriptions of the test specimen as it was set up for testing, as well as geometry of the specimen before and after testing. 


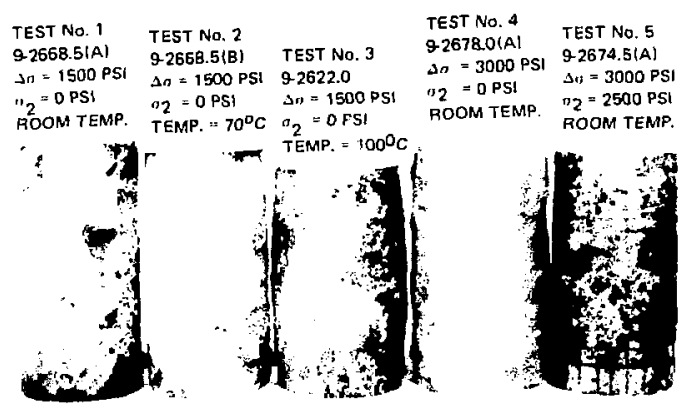

Figure 9. Photographs of Test Specimens 1 through 5 .

(a) Test 1: no visible cracks; (b) Test 2: few medium cracks between large crystals, minor cracking in large crystals;

(c) Test 3: few major cracks, man! hairline cracks; (d) Test 4: failed; (e) Test 5: no visible cracks.

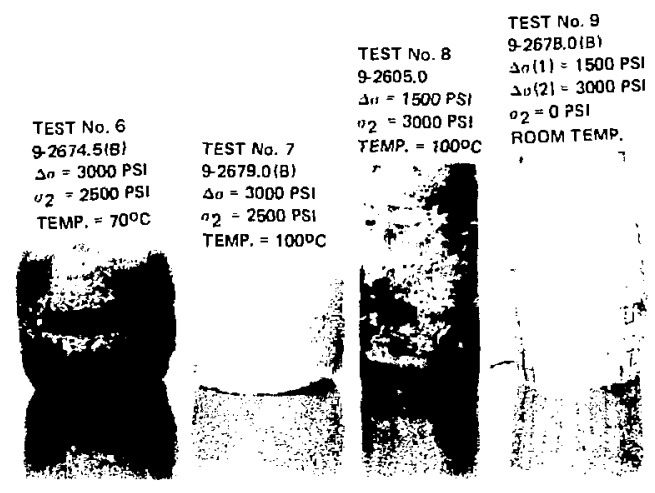

Figure 10. Photograph of Test Specinens 6 through 9.

(a) Test 6: major cracking on ridges, specimen well-deformed with no visible cracks; (b) Test 2: sank; (c) Test 8: minor hairline cricks; (d) rest 9: failed. 
TEST No. 10

g.2606.0(B)

$\triangle a(1)=1500 \mathrm{PS}$ $د n(2)=3000$ PS $n_{2}=2500 \mathrm{PSI}$ ROOM TEMP.

TEST No. 11 9.2679.01A $2 v=1500$ PSI $\mathrm{O}_{2}=\mathrm{OPSI}$ TEMP. $=1000^{\circ}$
TEST No. 12 TEST No. 13 9.2678.3(B) 92605.5(B) Lo(1) $=1500$ PSI $\quad I_{n}=4500$ PS $20(2)=3000$ PSI $112=2000$ PSI ${ }^{\prime \prime 2}=2500$ PSI ROOM TEMP.

TEST No. 14 g. $2678.7(8)$ $\Delta_{a}=6000 \mathrm{PSI}$ "I $2=1500$ PSI FOOM TEMP.

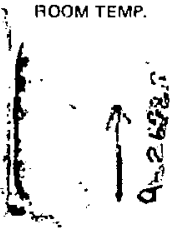

Figure 11. Photograph of Test Specimens 10 through 14.

(a) Test 10: large crystals show cracking, few hairline cracks throughout; (b) Test 11: major cracks, many medium and smal I cracks; (c) Test 12: some major cracks on ends, hairline cracks throughout; (d) Test 13: large crystals are cracked, no other visible cracks; (e) Test 14: major cracking on ends, large crystals cracked, many hairline cracks throughout.

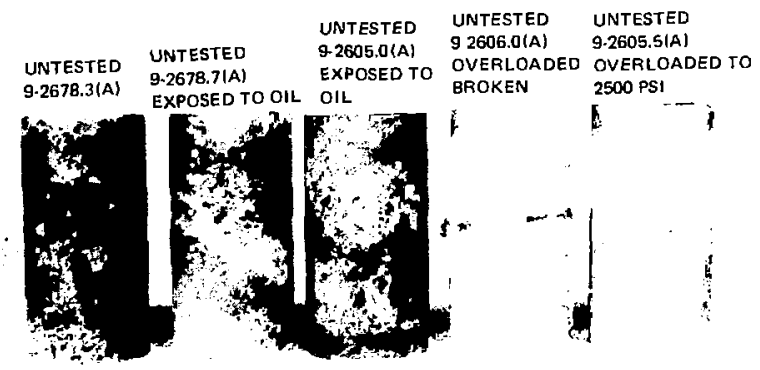

Figure 12. Photograph of Untested Specimens. 


\subsection{Activation Energy}

The purpose of this section is to present the results of activation energy calculations. Several experiments in this test matrix exhibited a constant, or nearly so, creep rate. The activation energy, $Q_{c}$ for rock salt can be calculated if the creep rate is known at two temperatures, $T_{1}$ and $T_{2}$. From Sherby and Burke (2), the equation is:

$$
\left.Q_{c}\right|_{\sigma, \varepsilon}=R \ln \left(\dot{\varepsilon}_{1} / \varepsilon_{2}\right) /\left(1 / T_{2}-1 / T_{1}\right)
$$

where:

$\dot{\varepsilon}_{1}$ and $\dot{\varepsilon}_{2}=$ axial strain rates at $T_{1}$ and $T_{2}$, respectively. $R=$ gas constant

In application of this equation to the experimental results, the strain-time plots were visually examined and steady state was assumed over portions of the plots which were linear. Table 7 summarizes the results obtained in this manner.

From the data contained in Table 7 , four calculations of activation energy have been made:

$$
\begin{aligned}
Q_{2,3} & =0,460 \mathrm{cal} / \mathrm{mole} \\
Q_{2,8} & =9,100 \mathrm{cal} / \mathrm{mole} \\
Q_{6,7} & =10,990 \mathrm{cal} / \mathrm{mole} \\
Q_{6,12} & =19,715 \mathrm{cal} / \mathrm{mole}
\end{aligned}
$$

Certainly this amount of data cannot be construed as conclusive, however, it is comforting to note that these activation energies are in the range of values published by le compte ( 3 ).

Though it is not practical to over-analyze minimal data, it is interesting to note correlation of these data to an equation from Heard (4):

$$
\varepsilon=A \exp \left(-Q_{C} / R T\right)_{\sigma} N
$$

where:

$$
\begin{aligned}
& \varepsilon \text { - steady state strain rate } \\
& A=\text { experimental constant } \\
& \sigma \text { - differential axial stress }
\end{aligned}
$$

Evaluation of the power on stress, $N$, for steady state creep is illustrated in Figure 13. 
As a result, $N$ is shown to be of the order of 6. From Heard (4), the value of $N=3,4.5$ and 6 , depending on the spectelc dislocation mechanism assumed to prevail. Aside from illustrating an apparent close fit to Equation 5 , no further analyses of these data are justified.

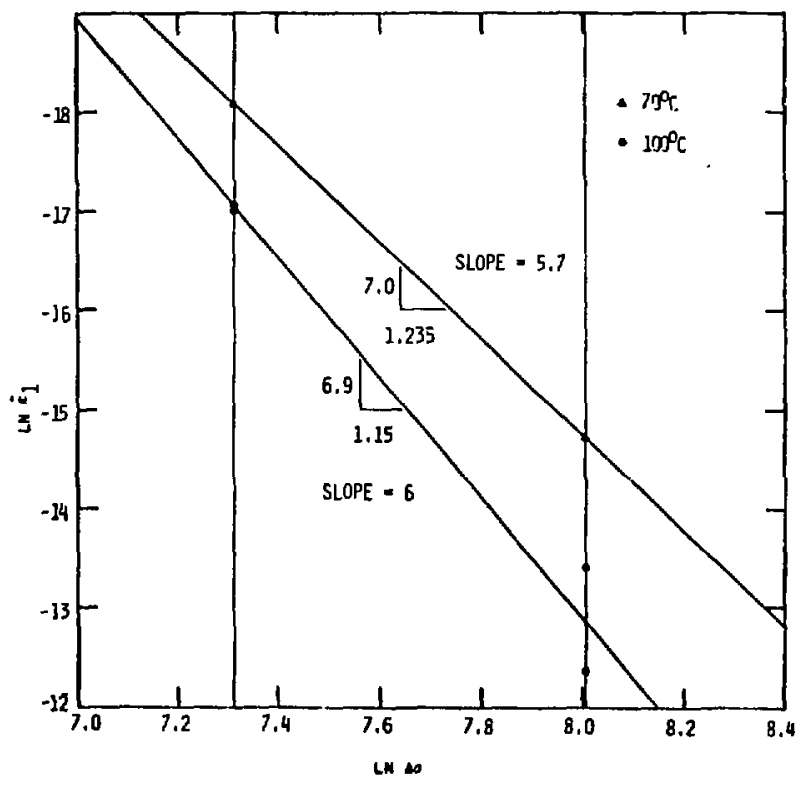

Figune 13. Pliot of LI \& 1 as a Function of LW a 
TABLE 7

STEADY STATE AXIAL CREEP

\begin{tabular}{|c|c|c|c|c|}
\hline $\begin{array}{l}\text { TEST } \\
\text { NO. }\end{array}$ & $\begin{array}{c}\text { TENP. } \\
\left.{ }^{\circ} \mathrm{C}\right)\end{array}$ & $\begin{array}{c}\text { DIFFERENTIAL } \\
\text { AXIAL STRESS/ } \\
\text { CONFINTNG PRESSURE } \\
\text { (PSI/PSI) }\end{array}$ & $\begin{array}{c}\text { TIME OVER WHICH } \\
\text { STEADY CREEP OCCURRED }\end{array}$ & $\begin{array}{c}\text { APPROXIMATE } \\
\text { STEADY STATE } \\
\text { CREEP RATE } \\
\left(E_{1} \text { seC. }^{-1}\right)\end{array}$ \\
\hline 2 & 70 & $1500 / 0$ & 140 to $500 \mathrm{HRS}$. & $1.4\left(10^{-8}\right)$ \\
\hline 3 & 100 & $1500 / 0$ & 110 to $360 \mathrm{HRS}$. & $3.8\left(10^{-8}\right)$ \\
\hline 4 & 24 & $3000 / 0$ & 1.4 to $5.0 \mathrm{HRS}$. & $1.0\left(10^{-6}\right)$ \\
\hline 6 & 70 & $3000 / 2500$ & 16 to 83 HRS. & $4.1\left(10^{-7}\right)$ \\
\hline 7 & 100 & $3000 / 2500$ & 8 to $20 \mathrm{HRS}$. & $1.5\left(10^{-6}\right)$ \\
\hline $\boldsymbol{\theta}$ & 100 & $1500 / 3000$ & 140 to 360 HRS. & $4.1\left(10^{-8}\right)$ \\
\hline 9 & 24 & $3000 / 0$ & 3 to $12 \mathrm{MIN}$. & $7.4\left(10^{-6}\right)$ \\
\hline 12 & 100 & $3000 / 2500$ & 6 to 12.5 HRS. & $4.2\left(10^{-6}\right)$ \\
\hline 14 & 24 & $6000 / 1500$ & 42 to 88 HRS. & $4.8\left(10^{-7}\right)$ \\
\hline
\end{tabular}




\subsection{Comparison with Previous Results}

Wany of the experiments in this investigation were conducted under similar stresses and temperatures to tests reported in Reference 1. Principal differences between these two test efforts are: (I) Specimens tested in this investigation were obtained from ERDA Hole No. 9 at 2605 to 2679 foot depth while those tested previously were from AEC Hole No. 8 at 2711 to 2722 foot depth; (2) Less time elapsed between field drilling and laboratory testing than previously and core from ERDA "9 was carefully protected from environmental effects; (3) Differential stress was corrected to account for specimen deformation in this work, whereas, previously the stress was not corrected; and (4) Temperature of the tests in this study were actual specimen temperatures rather than oil temperature. General characterization of the salt was similar; each group was relatively clean though ERDA "I9 salt contained minor amounts of clay impurities.

Finally, it is interesting to compare results. For that purpose, Table $B$ has been compiled which 1 ists strain rates obtained this year and rates of similar tests reported previously. The experiments conducted at room temperature, $24^{\circ} \mathrm{C}$, compare very favorably. The experiments conducted at $100^{\circ} \mathrm{C}$ illustrate the different rates attained by correcting differential stress opposed to not correcting. Also included in this difference is the effect of temperature differences, though thought to be much less significant.

Another way of presenting the difference between results of this experimental program and the previous program is by comparison of power laws which include differential stress and temperature dependence:

$$
\begin{array}{ll}
\varepsilon_{1}=1.693\left(10^{-39}, t^{0.4808} \sigma^{2.676} T^{10.17}\right. & \text { (Reference } 1 \text { ) } \\
\varepsilon_{I}=1.1\left(10^{-35}\right) t^{0.4556} \sigma^{2.475} \pi^{8.969} & \text { (Equation 4-a) }
\end{array}
$$

Generally, the strais dependence on time, differentlal stress and temperature are very comparable. However, the experimental coeffictent differs by four orders of magnitude. As far as application of these two equaions to calculate strain magnitudes at a given time ( $t \leq 10$ days), the results are surprisingly comparable. 
TABLE 8

COMPARISON OF EXDERINENTAL DATA FOR TESTS INCLUDED IN THIS REPORT AND FOR TESTS AS REPORTED IN REFERENCE I

\begin{tabular}{|c|c|c|c|c|c|c|}
\hline \multirow[b]{2}{*}{ TEST* } & \multirow{2}{*}{$\begin{array}{l}D A T E \\
(Y R)\end{array}$} & \multirow{2}{*}{$\begin{array}{c}\text { CONFINING } \\
\text { PRESSURE } \\
\text { (PSI) }\end{array}$} & \multicolumn{4}{|c|}{ AXIAL STRAIN RATES (SEC ${ }^{-1}$ ) AT VARTOUS TTMES* * } \\
\hline & & & $2 H R$ & $10 H R$ & $100 \mathrm{HR}$ & $200 \mathrm{HR}$ \\
\hline \multicolumn{7}{|c|}{ CONDUCTED AT $24^{\circ} \mathrm{C}$ AND $\triangle O=1500 \mathrm{PSI}$} \\
\hline $\begin{array}{c}R C-1(\text { STG } 1) \\
R C-3(\text { STG } 1) \\
1 \\
9(\text { STG } 1) \\
10(\text { STG } 1)\end{array}$ & $\begin{array}{l}76 \\
76 \\
77 \\
77 \\
77\end{array}$ & $\begin{array}{r}500 \\
2000 \\
0 \\
0 \\
2500\end{array}$ & $\begin{array}{l}5.62\left(10^{-8}\right) \\
4.58\left(10^{-8}\right) \\
4.50\left(10^{-8}\right) \\
3.69\left(10^{-8}\right) \\
3.35\left(10^{-8}\right)\end{array}$ & $\begin{array}{l}1.80\left(10^{-8}\right) \\
1.56\left(10^{-8}\right) \\
1.58\left(10^{-8}\right) \\
1.29\left(10^{-8}\right) \\
1.19\left(10^{-8}\right)\end{array}$ & $\begin{array}{l}3.55\left(10^{-9}\right) \\
3.34\left(10^{-9}\right) \\
3.53\left(10^{-9}\right) \\
2.89\left(10^{-9}\right) \\
2.72\left(10^{-9}\right)\end{array}$ & $\begin{array}{l}2.16\left(10^{-9}\right) \\
2.10\left(10^{-9}\right) \\
2.25\left(10^{-9}\right) \\
1.84\left(10^{-9}\right) \\
1.74\left(10^{-9}\right)\end{array}$ \\
\hline \multicolumn{7}{|c|}{ CUNDIICTED AT $100^{\circ} \mathrm{C}$ AND AO $=1500 \mathrm{PSI}$} \\
\hline $\begin{array}{l}R C-2 \text { (STG } 1) \\
R C-5(S T G 1) \\
3 \\
11 \\
12(S T G 1)\end{array}$ & $\begin{array}{l}76 \\
76 \\
77 \\
77 \\
77\end{array}$ & $\begin{array}{r}500 \\
2000 \\
0 \\
0 \\
2500\end{array}$ & $\begin{array}{l}2.69\left(10^{-7}\right) \\
3.77\left(10^{-7}\right) \\
5.27\left(10^{-7}\right) \\
5.56\left(10^{-7}\right) \\
3.40\left(10^{-7}\right)\end{array}$ & $\begin{array}{l}1.22\left(10^{-7}\right) \\
1.54\left(10^{-7}\right) \\
2.12\left(10^{-7}\right) \\
2.83\left(10^{-7}\right) \\
1.79\left(10^{-7}\right)\end{array}$ & $\begin{array}{l}3.91\left(10^{-8}\right) \\
4.30\left(10^{-8}\right) \\
5.78\left(10^{-8}\right) \\
1.08\left(10^{-7}\right) \\
7.12\left(10^{-8}\right)\end{array}$ & $\begin{array}{l}2.78\left(10^{-8}\right) \\
2.52\left(10^{-8}\right) \\
3.91(1 .-8) \\
8.05\left(10^{-8}\right) \\
5.40\left(10^{-8}\right)\end{array}$ \\
\hline \multicolumn{7}{|c|}{ CONDUCTED AT $24^{\circ} \mathrm{C}$ AND AO $=3000 \mathrm{PSI}$} \\
\hline $\begin{array}{c}R C-1(\text { STG } 2) \\
R C-3(\text { STG } 2) \\
R C-4(\text { STG } 1) \\
4 \\
9(\text { STG } 2) \\
5 \\
10(\text { STG } 2)\end{array}$ & $\begin{array}{l}76 \\
76 \\
76 \\
77 \\
77 \\
77 \\
77\end{array}$ & $\begin{array}{r}500 \\
2000 \\
2000 \\
0 \\
0 \\
2500 \\
2500\end{array}$ & $\begin{array}{c}2.64\left(10^{-7}\right) \\
2.39\left(10^{-7}\right) \\
2.20\left(10^{-7}\right) \\
1.10\left(10^{-6}\right) \\
\text { RUPTURED } \\
1.92\left(10^{-7}\right) \\
2.10\left(10^{-7}\right)\end{array}$ & $\begin{array}{c}1.11\left(10^{-7}\right) \\
8.74\left(10^{-8}\right) \\
3.58\left(10^{-8}\right) \\
\text { RUPTURED } \\
=- \\
9.40\left(10^{-8}\right) \\
9.42\left(10^{-8}\right)\end{array}$ & $\begin{array}{c}3.24\left(10^{-8}\right) \\
2.07\left(10^{-8}\right) \\
2.21\left(10^{-8}\right) \\
- \\
3.39\left(10^{-8}\right) \\
2.99\left(10^{-8}\right)\end{array}$ & $\begin{array}{c}2.23\left(10^{-8}\right) \\
1.34\left(10^{-8}\right) \\
1.47\left(10^{-8}\right) \\
-- \\
- \\
2.49\left(10^{-8}\right) \\
2.12\left(10^{-8}\right)\end{array}$ \\
\hline \multicolumn{7}{|c|}{ CONDUCTED AT $100^{\circ} \mathrm{C}$ AND DO $=3000$ PSI } \\
\hline $\begin{array}{c}R C-2(\text { STG 2) } \\
R C-5(\text { STG 2) } \\
R C-6(\text { STG } 1) \\
7 \\
12(\text { STG 2) }\end{array}$ & $\begin{array}{l}76 \\
76 \\
76 \\
77 \\
77\end{array}$ & $\begin{array}{r}500 \\
2000 \\
2000 \\
2500 \\
2500\end{array}$ & $\begin{array}{l}2.01\left(10^{-6}\right) \\
1.87\left(10^{-6}\right) \\
2.10\left(10^{-6}\right) \\
3.20\left(10^{-6}\right) \\
5.29\left(10^{-6}\right)\end{array}$ & $\begin{array}{l}9.00\left(10^{-7}\right) \\
8.39\left(10^{-7}\right) \\
8.44\left(10^{-7}\right) \\
1.91\left(10^{-6}\right) \\
2.86\left(10^{-6}\right)\end{array}$ & $\begin{array}{l}2.84\left(10^{-7}\right) \\
2.71\left(10^{-7}\right) \\
2.29\left(10^{-7}\right) \\
\ldots \\
-\end{array}$ & $\begin{array}{c}2.01\left(10^{-7}\right) \\
1.93\left(10^{-7}\right) \\
1.55\left(10^{-7}\right) \\
- \\
--\end{array}$ \\
\hline \multicolumn{7}{|c|}{ CONDUCTED AT $24^{\circ} \mathrm{C}$ AHD DO $=4500 \mathrm{PST}$} \\
\hline $\begin{array}{c}R C-1(\operatorname{STG} 3) \\
R C-3(S T G \quad 3) \\
R C-4(S T G \quad 2) \\
13\end{array}$ & $\begin{array}{l}76 \\
76 \\
76 \\
77\end{array}$ & $\begin{array}{r}500 \\
2000 \\
2000 \\
2000\end{array}$ & $\begin{array}{l}7.42\left(10^{-7}\right) \\
7.06\left(10^{-7}\right) \\
6.67\left(10^{-7}\right) \\
7.38\left(10^{-7}\right)\end{array}$ & $\begin{array}{l}3.03\left(10^{-7}\right) \\
2.67\left(10^{-7}\right) \\
2.65\left(10^{-7}\right) \\
3.35\left(10^{-7}\right)\end{array}$ & $\begin{array}{l}8.38\left(10^{-8}\right) \\
6.62\left(10^{-8}\right) \\
7.08\left(10^{-8}\right) \\
1.08\left(10^{-7}\right)\end{array}$ & $\begin{array}{l}5.70\left(10^{-8}\right) \\
4.36\left(10^{-8}\right) \\
4.76\left(10^{-8}\right) \\
7.69\left(10^{-8}\right)\end{array}$ \\
\hline
\end{tabular}

- Nomenclature RC-X refers to tasts docunented in Reference 1 . Information in parentheses, eg: $\left(S^{\prime} G-X\right)$, refers to stago of test 'here multiple stage creep tests were conducted on a single specimen.

** Calculated from indivigiual bost fit equation (Section 4.2) 


\section{CONCLUDING REMARKS}

This report has presented the results of seventeen ereep experiments completed on two-inch diameter rock salt specimens from ERDA Hole "to in southeastem New Mexico. The results also include limited triaxial compression data as a result of stress application to creep levels. The vast majority of the report discusses and presents axial and lateral strain behavior as a result of creeping under conditions of differential axial stresses ranging from 1500 to 6000 psi, lateral stresses ranging from 0 to $3000 \mathrm{psi}$, and temperatures from ambient laboratory temperature to $100^{\circ} \mathrm{C}$. Analyses of the test data are primarily in terms of curve fits to experimental data.

Quasi-static stress application to stress levels desired for creep experiments yielded relationships of differential axial stress as a function of strain. Axial strains were generally of the order of one percent and less. Moduli of deformation (determined by integration) ranged from 300,000 to 4,000,000 psi over the differential stress range of 0 to $1500 \mathrm{psi}$. In general, moduli decreased with increase in temperature. Principal strain ratio variation during guasi-static compression generally trended toward 0.5. At the end of quasi-static stress application, the principal strain ratio ranged between 0.33 to 0.48 and averaged 0.42 .

All tests exhibited transient creep to various degrees; two experiments resulted in rupture; and some experiments exhibited apparent stiady-state creep deformation which, in a few cases, accelerated prior to termination. The data are too limited to justify comprehensive analyses of primary, secondary and tertiary creen. The individual creep experiments were fit to power law forms of $\varepsilon_{1,2}=x t^{n}$ over the transient portions. Sunsequentlu, a regression analyses yielded an equation fitting axial creep data to a function of time, $t$, differential stress, $\sigma$, and temperature, $T$. The resultant equation is:

$$
\varepsilon_{1}=1.1\left(10^{-35}\right) t^{0.4656} \sigma^{2.475} T^{8.969}
$$

simtlar regression was not performed on the lateral strain data. However, on an Individual basis, lateral strain was shown to track axlal strain on d qualitatively identical curve. Actual principal strain ratios during croep tests ranged from 0.42 to 0.71 , but for the mst part, we re nearl! equal to 0.5 . Principal strain ratios generally exhibited a gradual increase as the test pmgressed. 
This testing program incorporated several refinements (improvements) as a result of last year's testing program. These jmprovements began with much improved sample preparation and osntinued through various changes in the experimental procedure. Broad comparisons of the previous results and these results have been incorporated in the text. 


\section{LIST OF REFERENCES}

(1) Hansen, Francis D.: "Triaxial, Quasi-Static Compression and Creep Behavior of Bedded Salt from Southeastern New Mexico", Technical Memorandum Report RSI-0055, Prepared for Sandia Laboratories operated by Sandia Corporation for the Energy Research and Development Administration, June 3, 1977.

(2) Sherby O. D. and Burke, P. M.: "Mechanical behavior of Crystalline Solids at Elevated Temperature", Progr. Metal Sci., Vol. 13, pP. 325-390, 1968 .

(3) Le Compte, P.: "Creep in Rock Salt", I. Geol., Vol. 73, pp. 469-484, 1965.

(4) Heard, H. C.: "Steady-state Flow in Polycrystalline Halite at Pressures of 2 Kilobars", Am. Geophys. Un. Mono., Vol. 16, 191-209, 1972. 


\section{APPENDIX A}

PLOTS OF DIFFERENTIAL STRESS

APPLICATION TO CREEP STAGE

FIGURE NO.

PAGE NO.

$A-1$

A-2

A-3

$A-4$

A- 5

A- 6

A-7

$A-8$

A-9

$A-10$

$A-11$

A -12

A- 13

$A=14$

A-15

$\lambda-16$

Differential Axial Stress as a Function of Axial strain, Test 1

Differential Axial Stress as a Function of Axial strain, Test 2

Differential Axial stress as a Function of Axial Strain, Test 3

Differontial Axial Stress as a Function of Axial strain, Test 4

Differential Axial Stress as a Function of Axial and Lateral strain, Test 5

Differential Axial stress as a Function of Axial and Laterul Strain, Test 6

Differential Axial Stress as a Function of Axial and Lateral Strain, Test 7

Differential Axial stress as a Function of Axial and Lateral Strain, Test $g$

Differential Axial Stress as a Function of Axial strain, Test 9, Stage 1

Differential Axial stress as a Function of Axial Strain, Test 9, Stage 2

Differential Axial Stress as a Function of Axial and Lateral Strain, Test 10, Stage 1

Differential Axial Stress as a Function of Axial and Lateral Strain, Test 10, Stage 2

Differential $\lambda x i a l$ Stress as a Function of Axial strain, rest 11 , Stage 1

Differential Axial stress as a Function of Axial strain, Test 11, stage 2 


\section{APRENDIX A (CONT'D)}

FIGURE NO.

$A-17$

$A-18$

\section{PLOTTED PARAMETERS}

Differential Axial Stress as a Function of Axial and Lateral Strain, Test 13

Differential Axial Stress as a Function of Axial and Lateral Strain, Test 14
PAGE NO. 


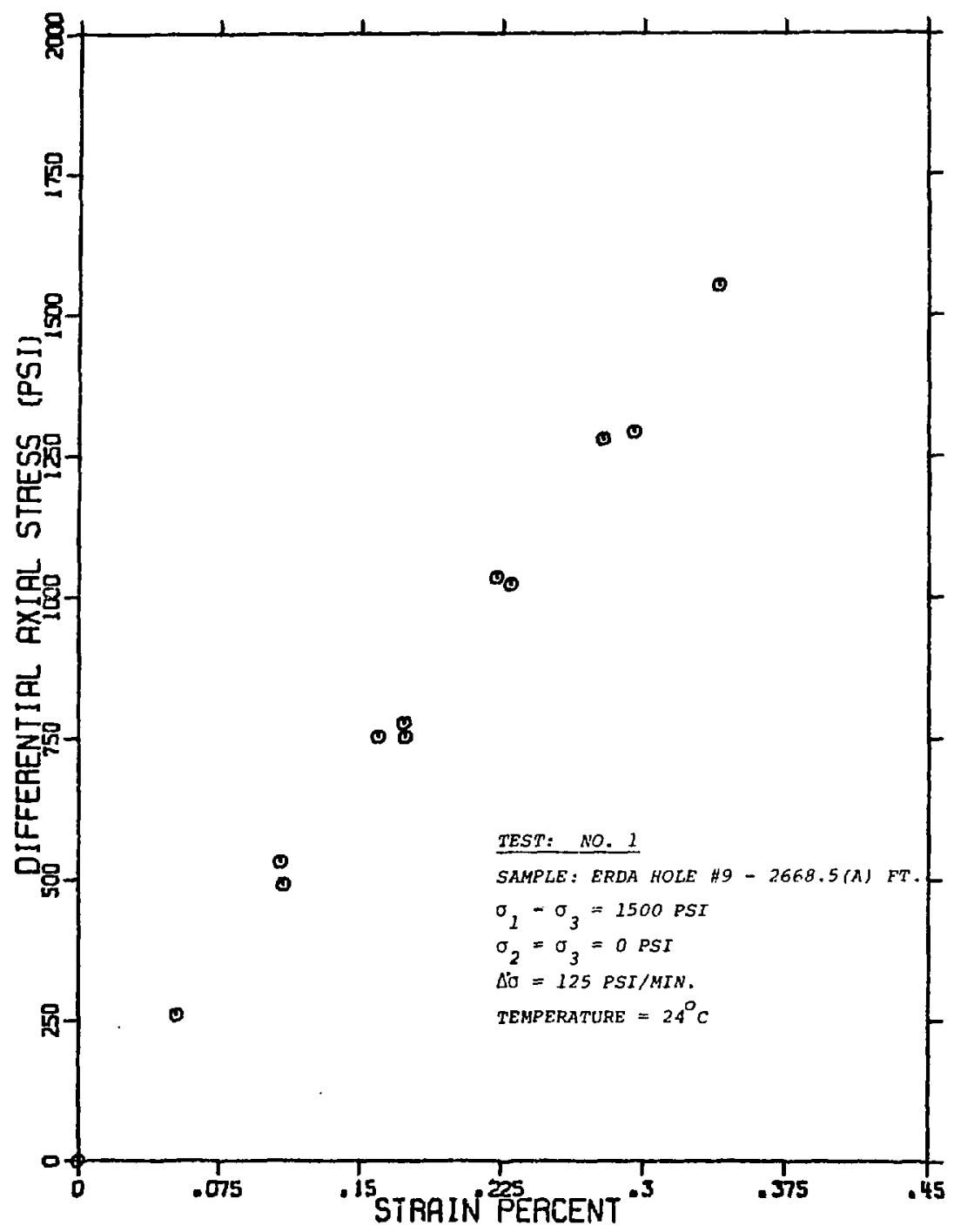

Figure A-1: Difforential Axial stress as a Function of Axial strain, Test. 1 . 


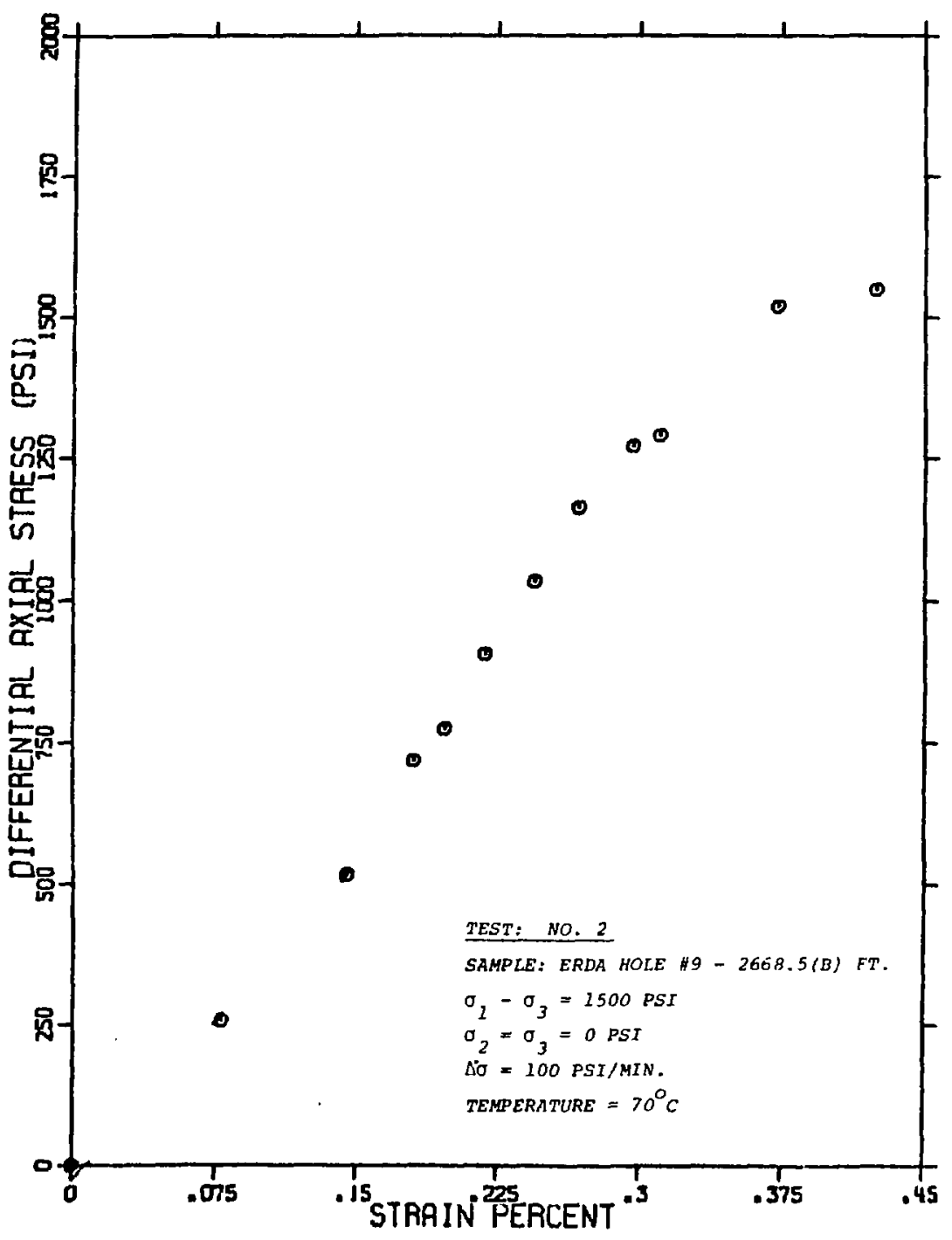

Figure A-2. Differential Axial stress as a Function of axial strán, Test 2. 


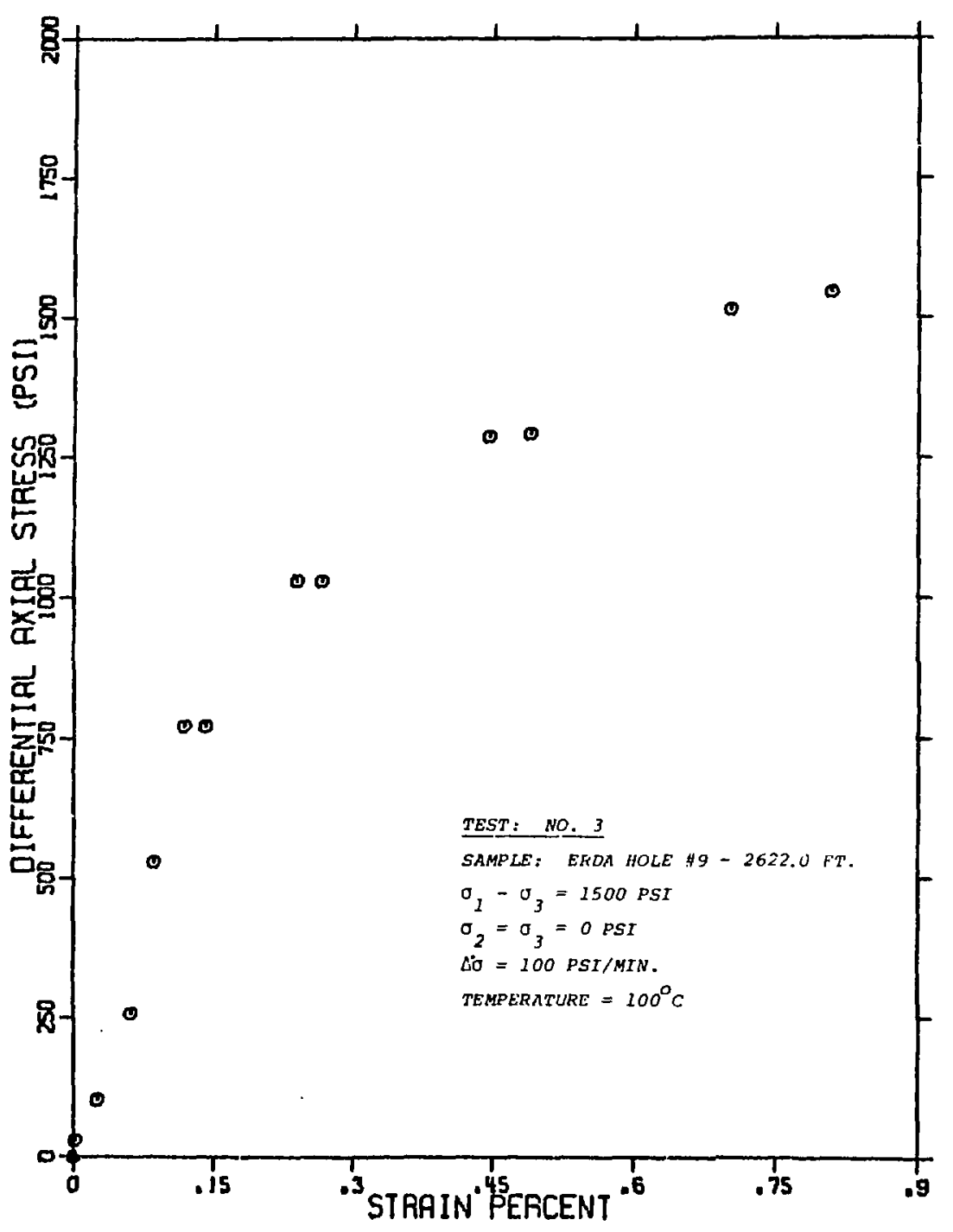

Figure A-3. Differential Axial stress as a Function of Axial strain, rest 3. 


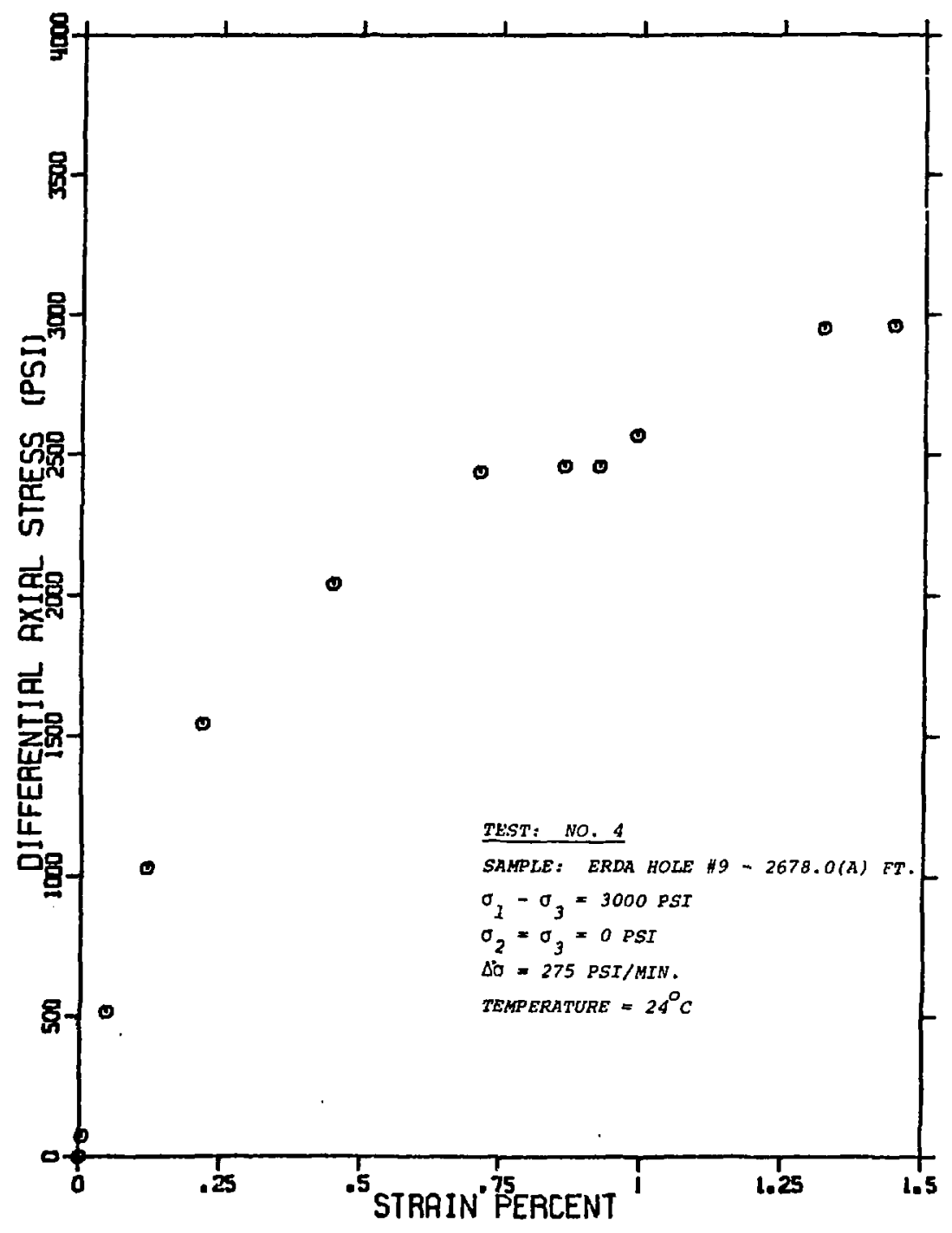

Figure A-4. Differential Axial Stress as a Function of Axial Strain, Test 4 . 


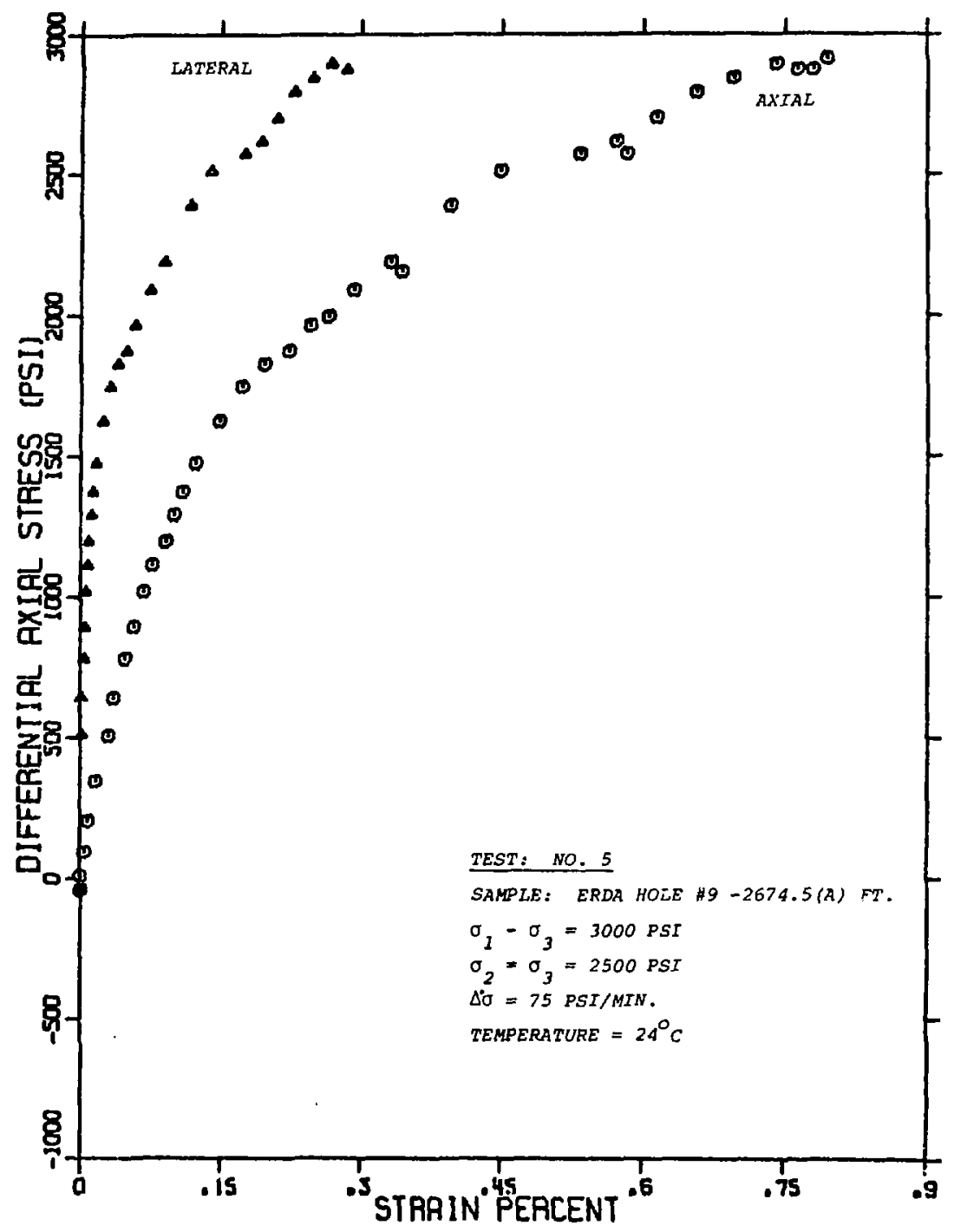

Figure A-5. Differential Axial Stress as a Function of Axial and Lateral straln, Test 5. 


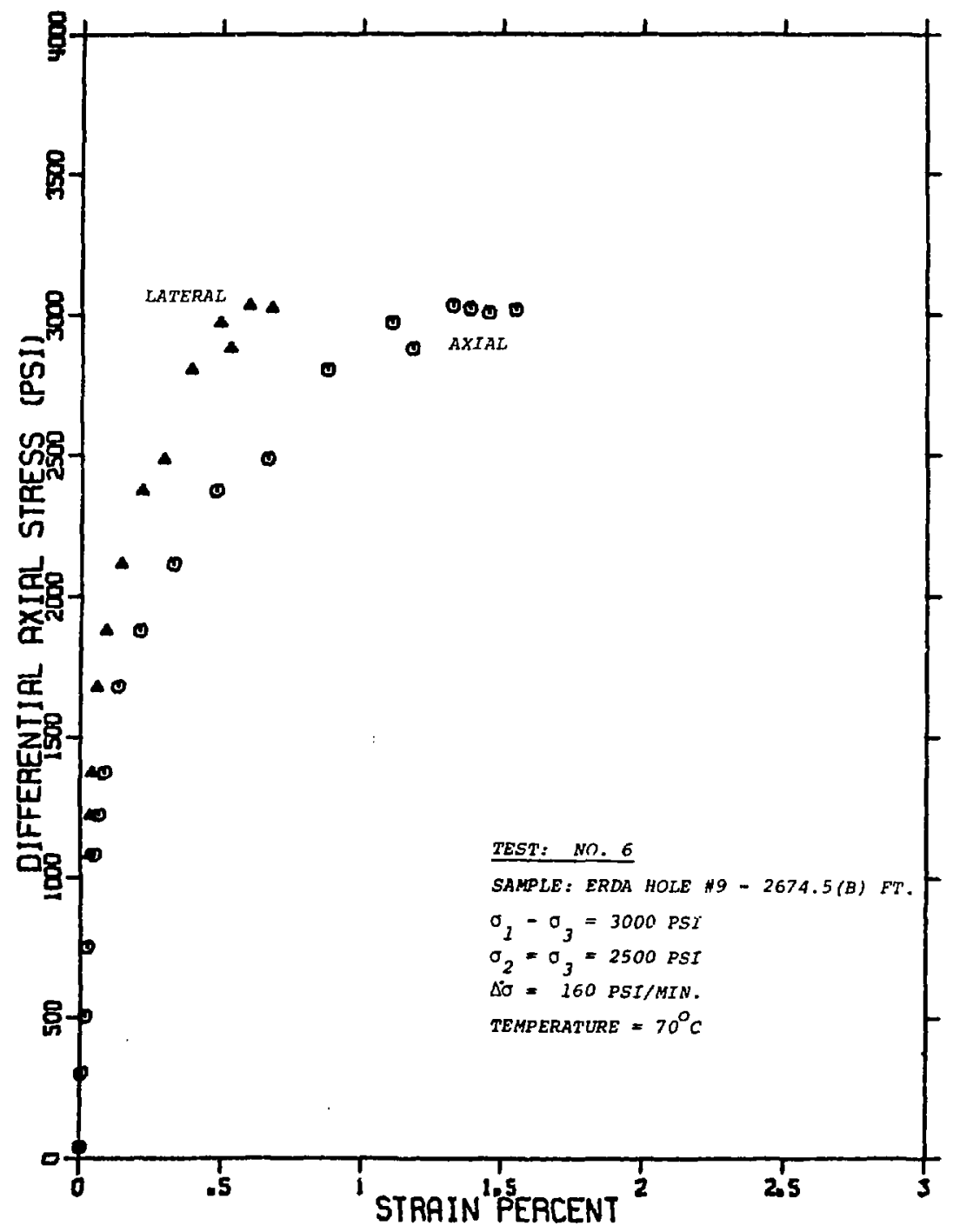

Figure $n-G$. Differential Axial stress as a function of Axial and Lateral strain, Test 6. 


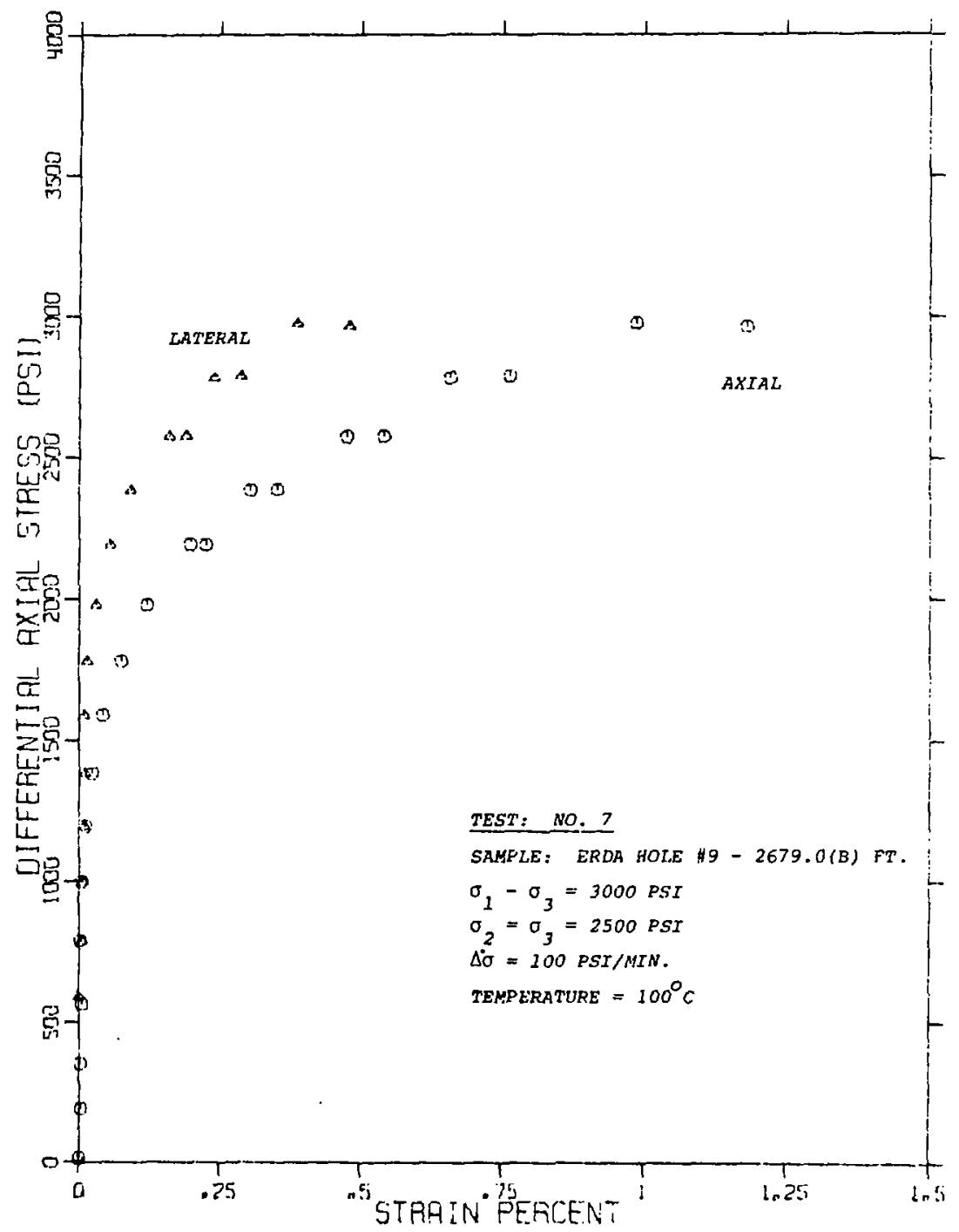

Figure A-7. Differential Axial Stress as a function of Axial and Lateral strain, Test 7.

51 


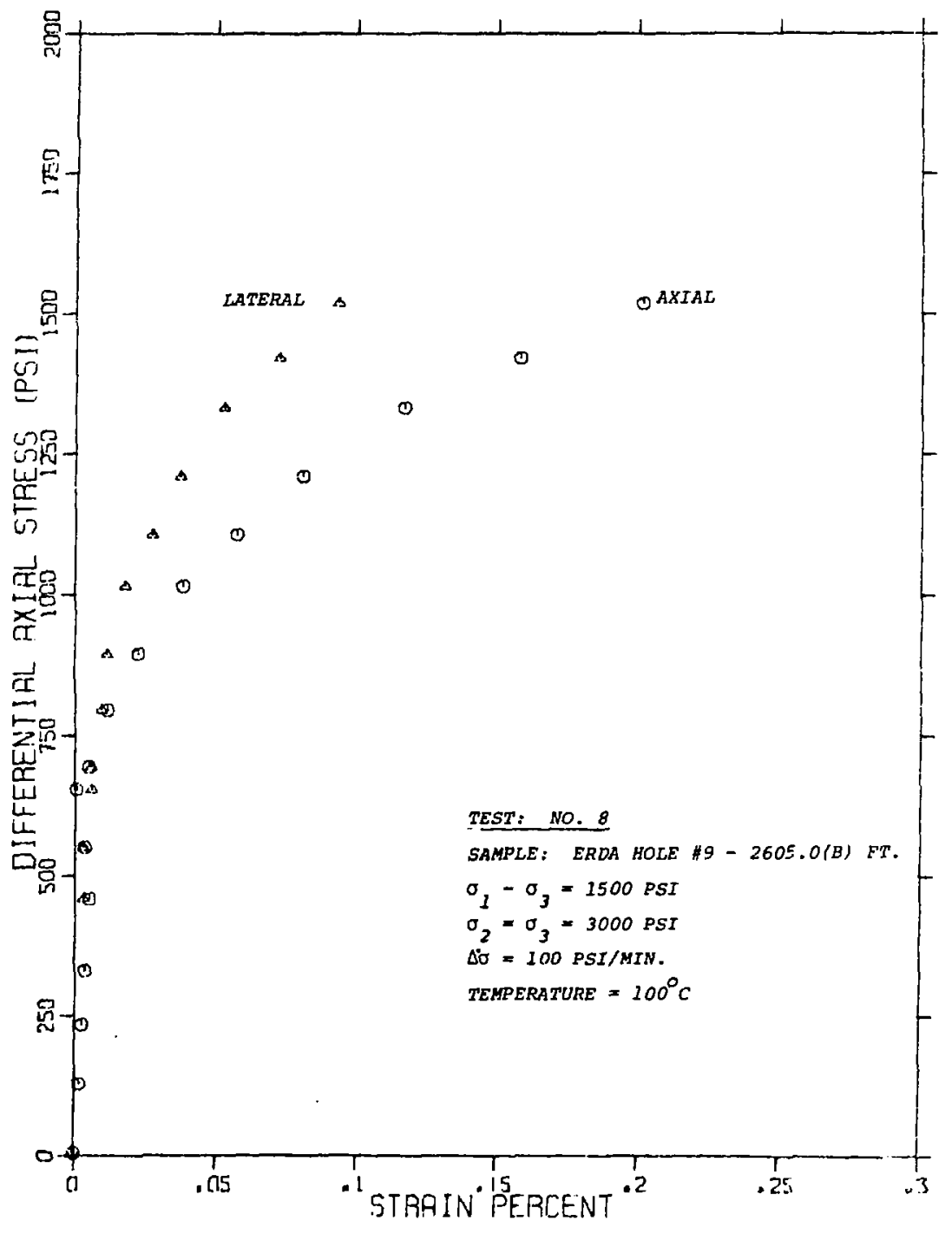

Figure A-8. Differential Axial Stress as a Function of Axial and Lateral strain, Test 8 . 


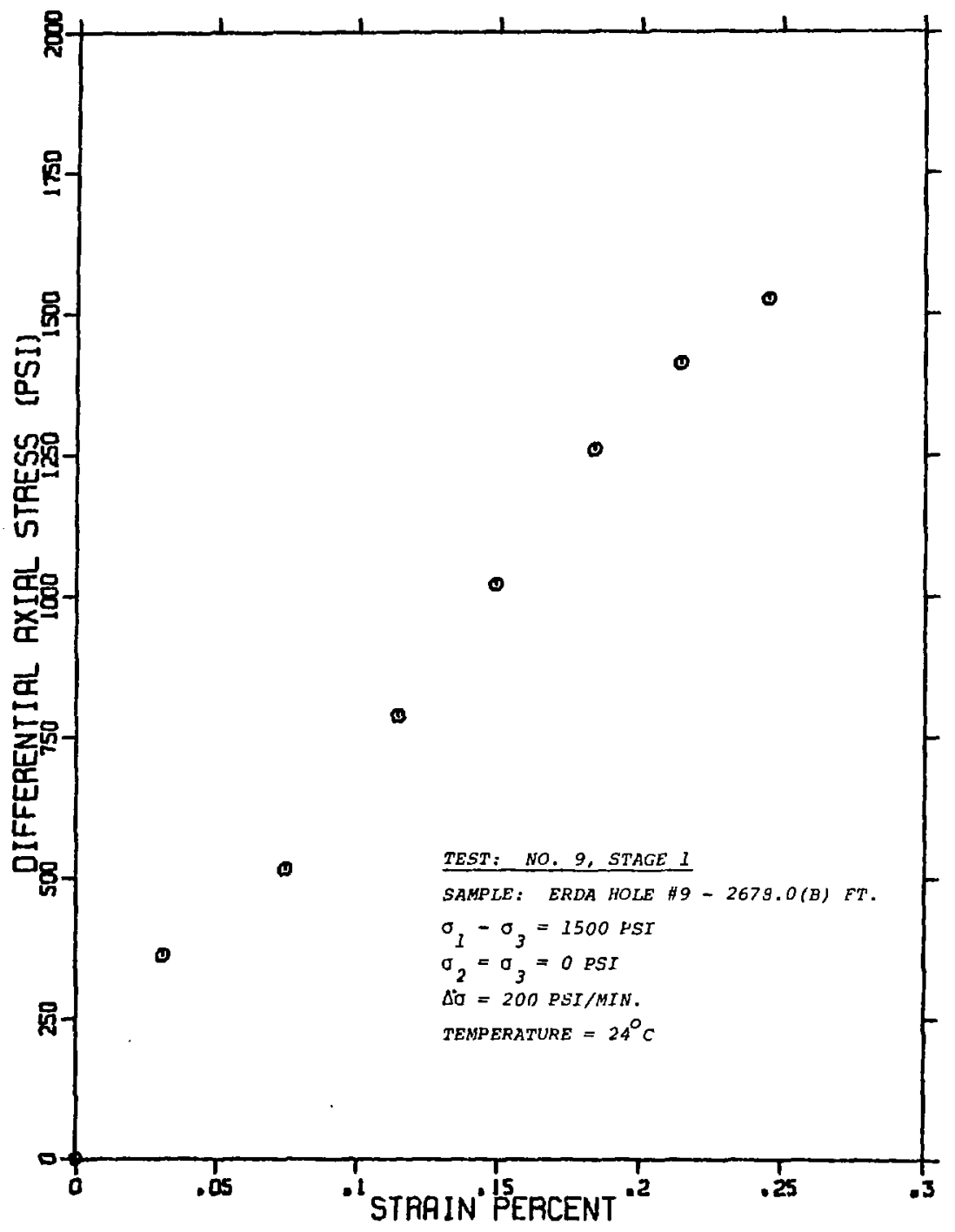

Figure A-9. Difforential Axial Stress as a Function of Axial strain, lest 9, Stage 1 . 


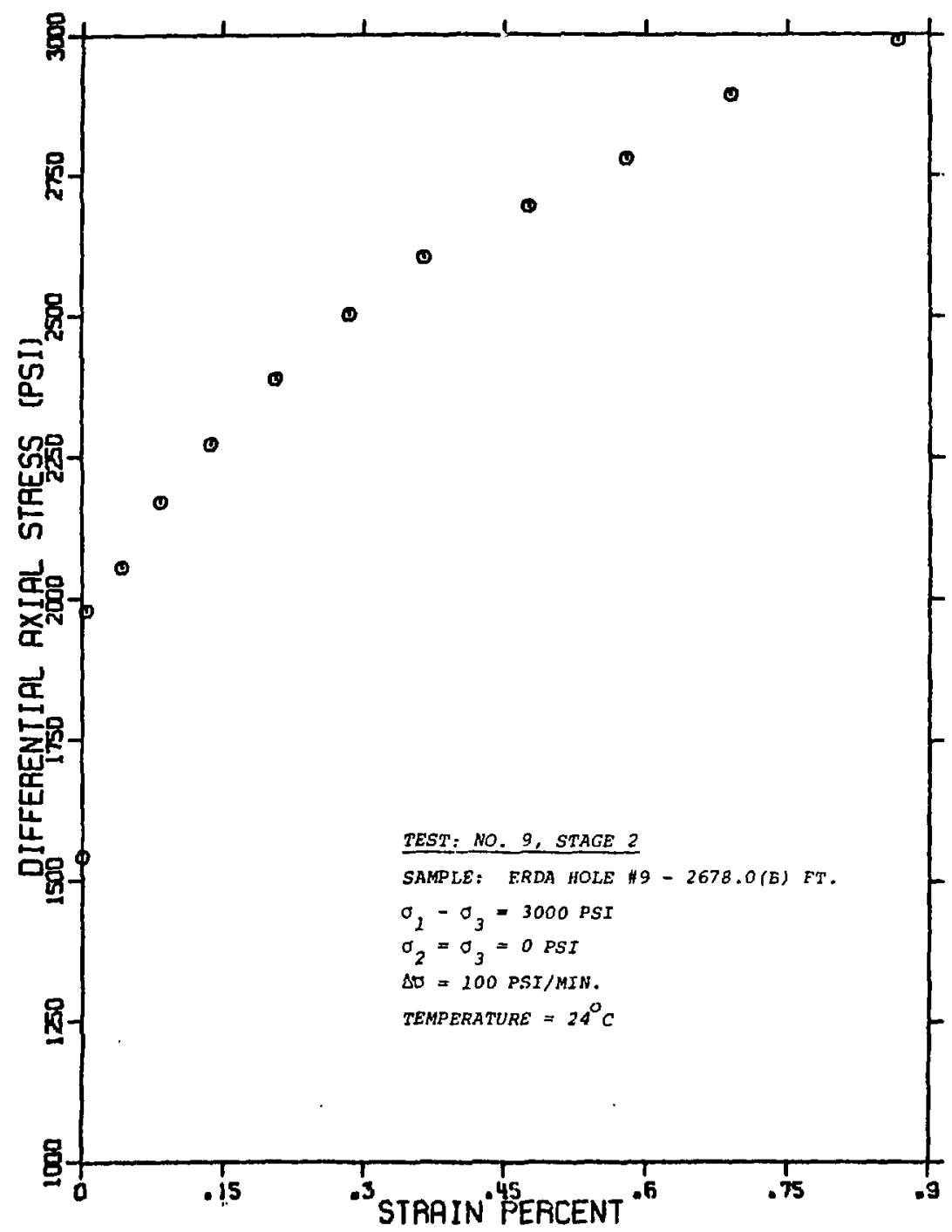

Figuro $\mathrm{n-30.}$ Differontial Axial stress as a function of Axial strain, Test 9, stage 2. 


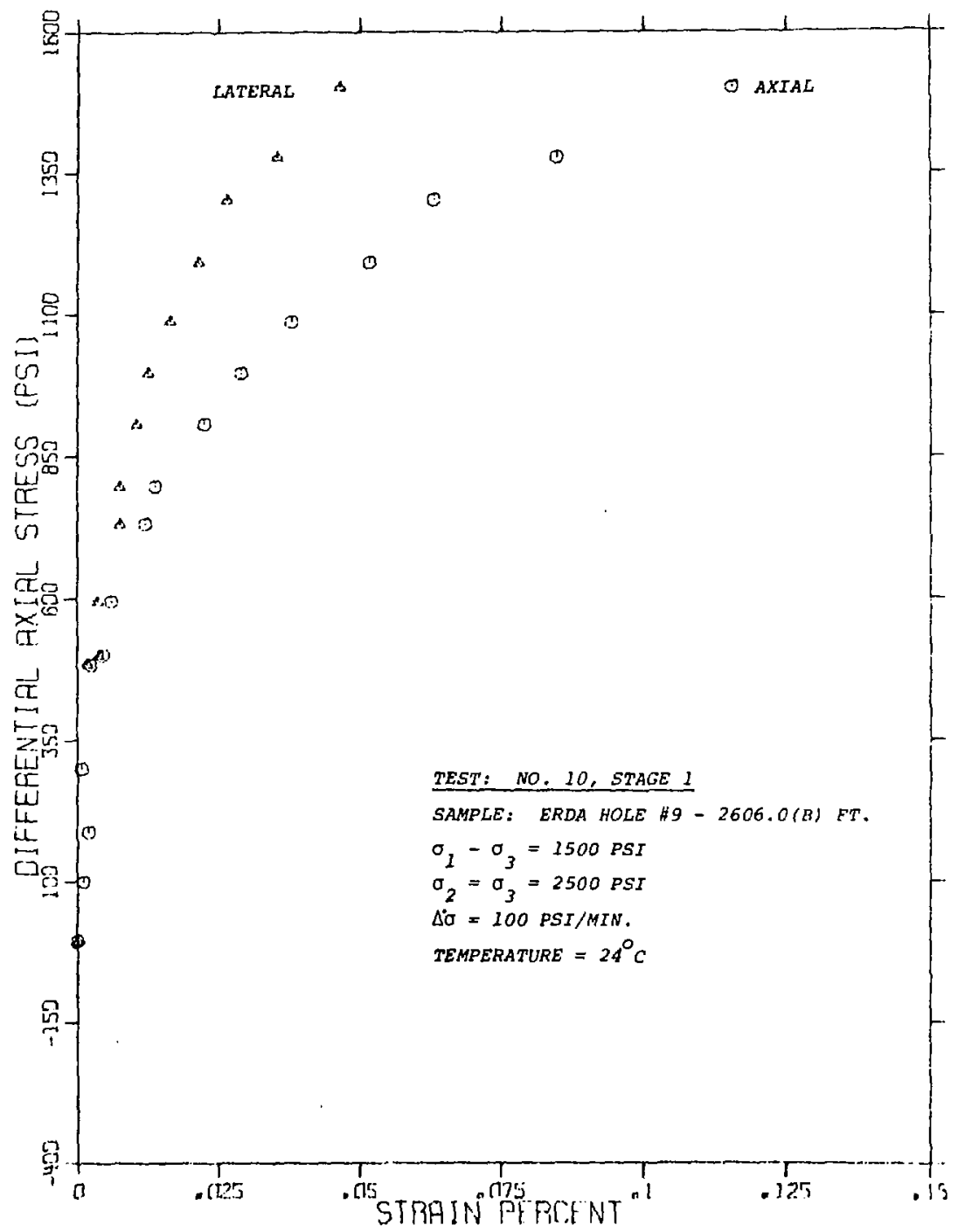

Figure A-11. Differential Axial stress as a Function of Axial and Latoral strain, pest 10, stạe 2. 


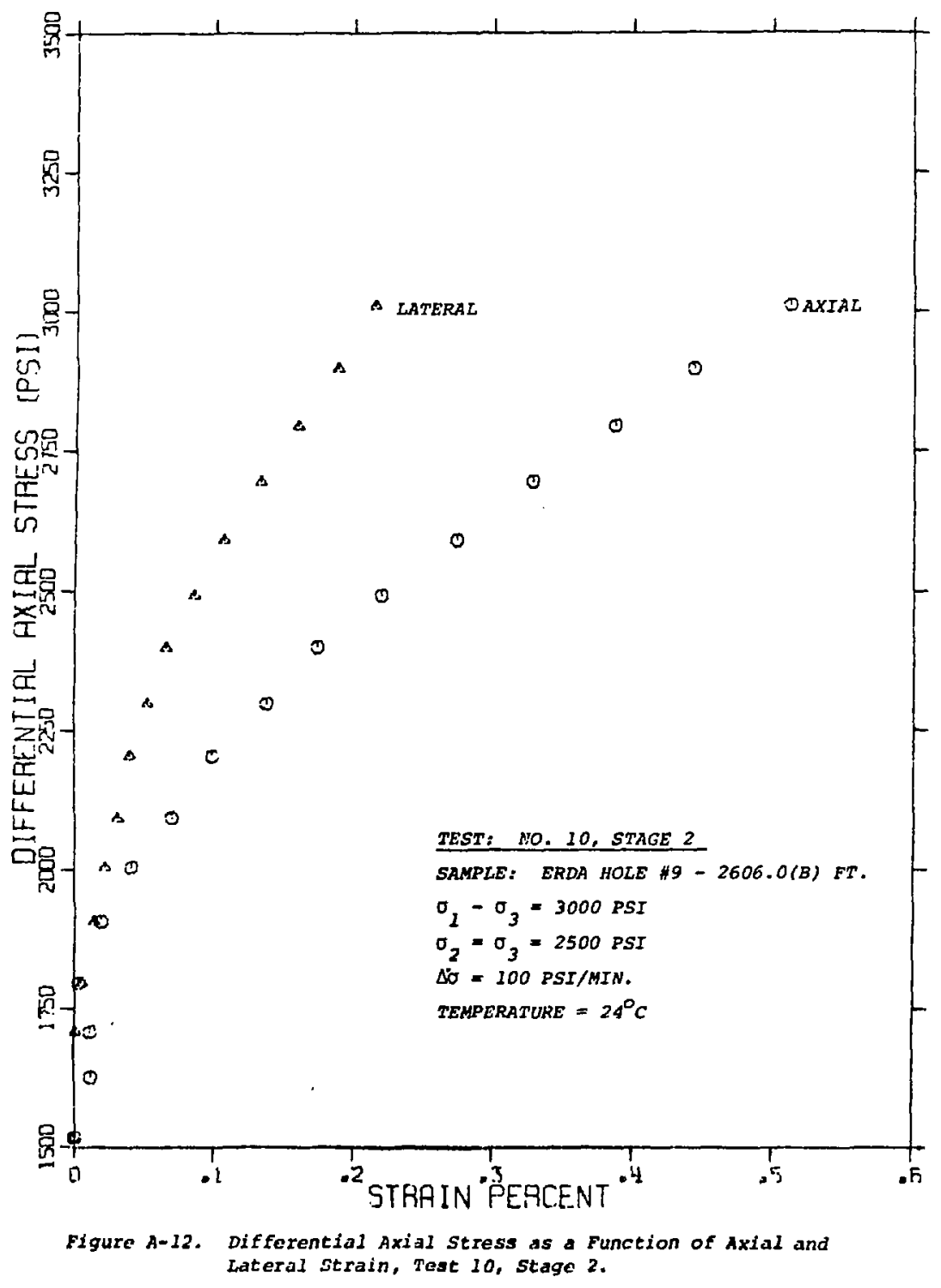




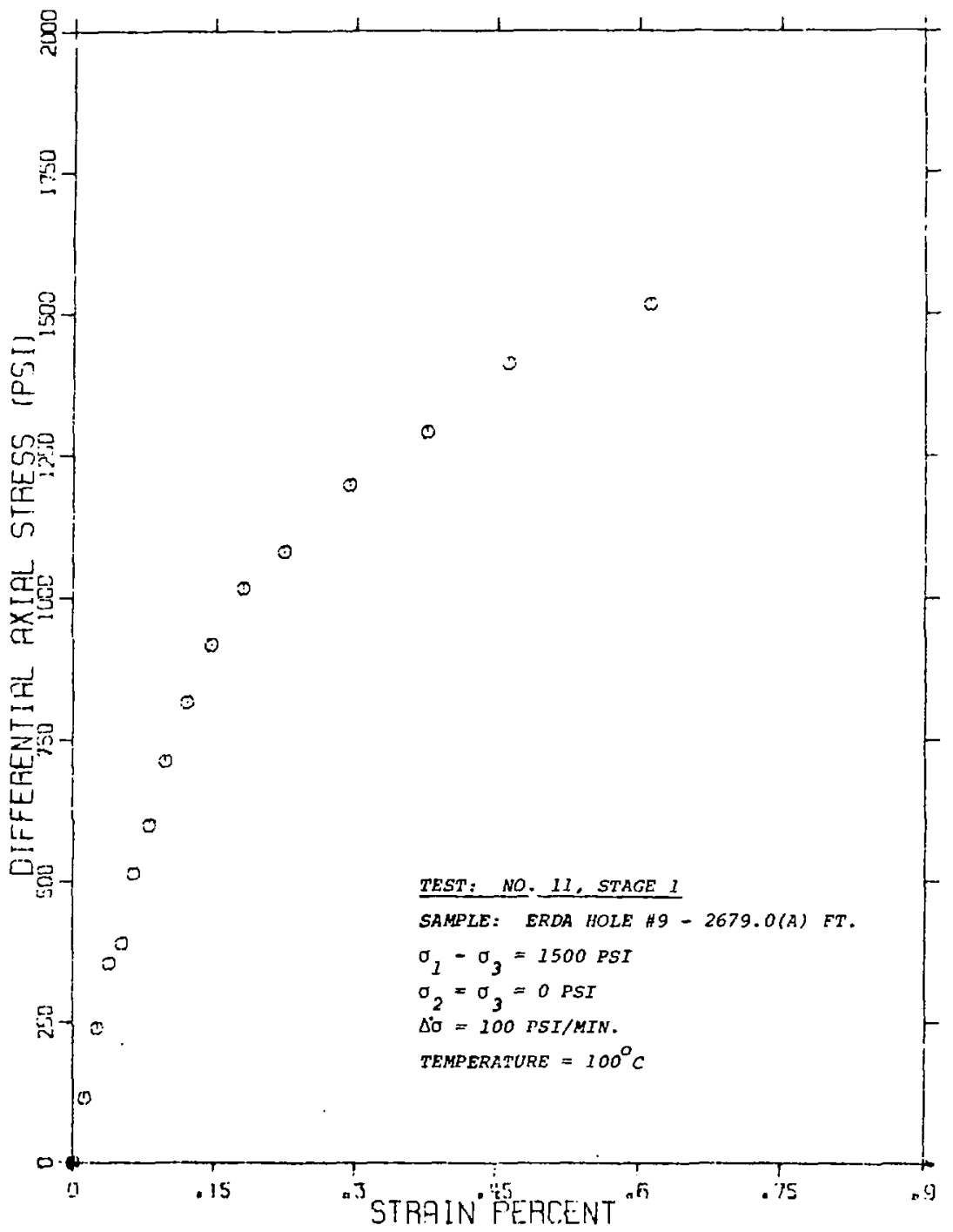

Figure A-13. Dlfferential Axial stress as a Function of Axial strain, Tost 11 , stiage 1 . 


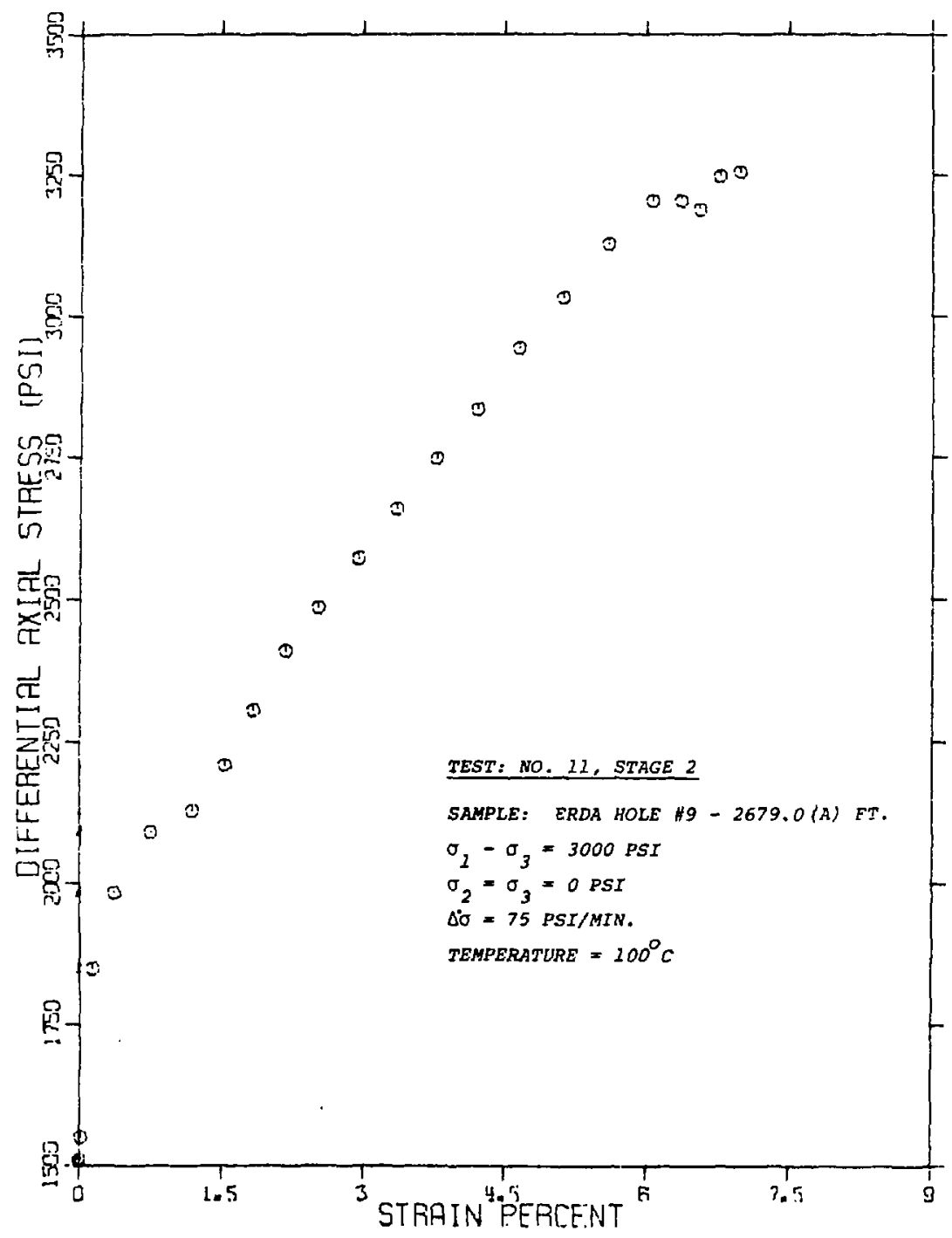

Flgure A-14. Differential Axial Stress as a Function of Axial Strain, Test 11, Stage 2. 


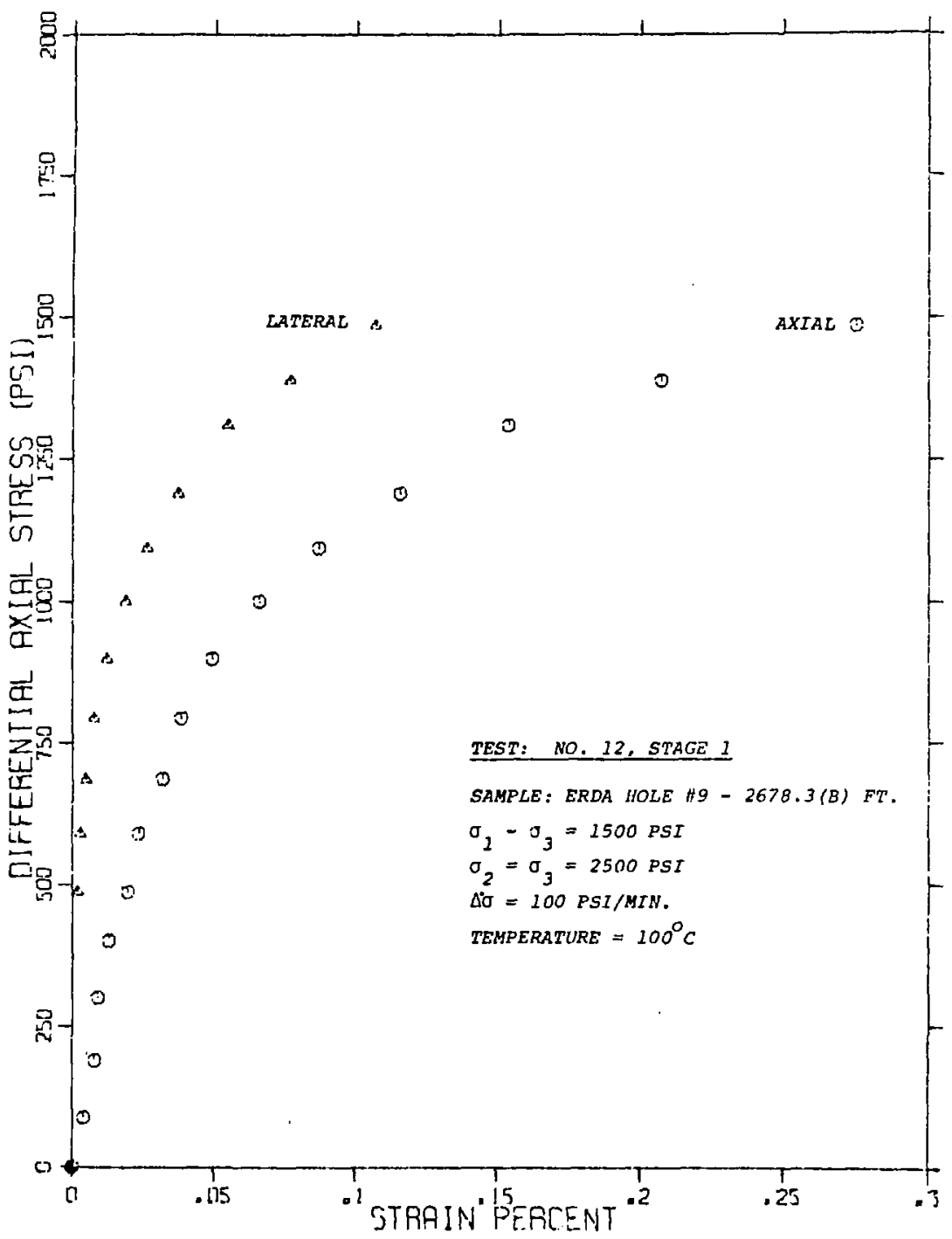

Figure A-15. Differential Axial Stress as a function of Axial and Lateral Strain, J'est 12. Stage 1. 


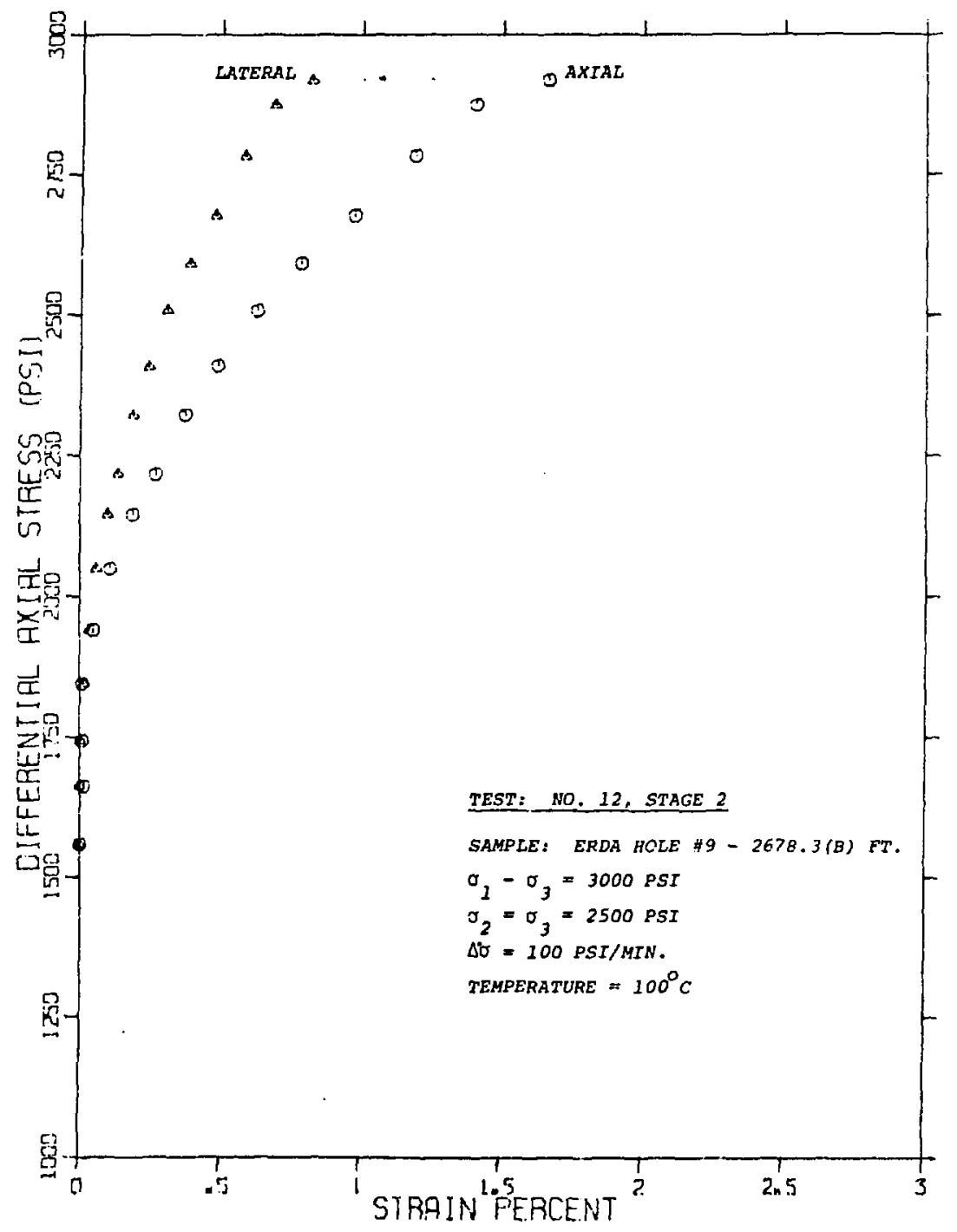

Plguro A-16. Differential Axial stress as a Function of Axlal and Lateral strain, Test 12, stage 2. 


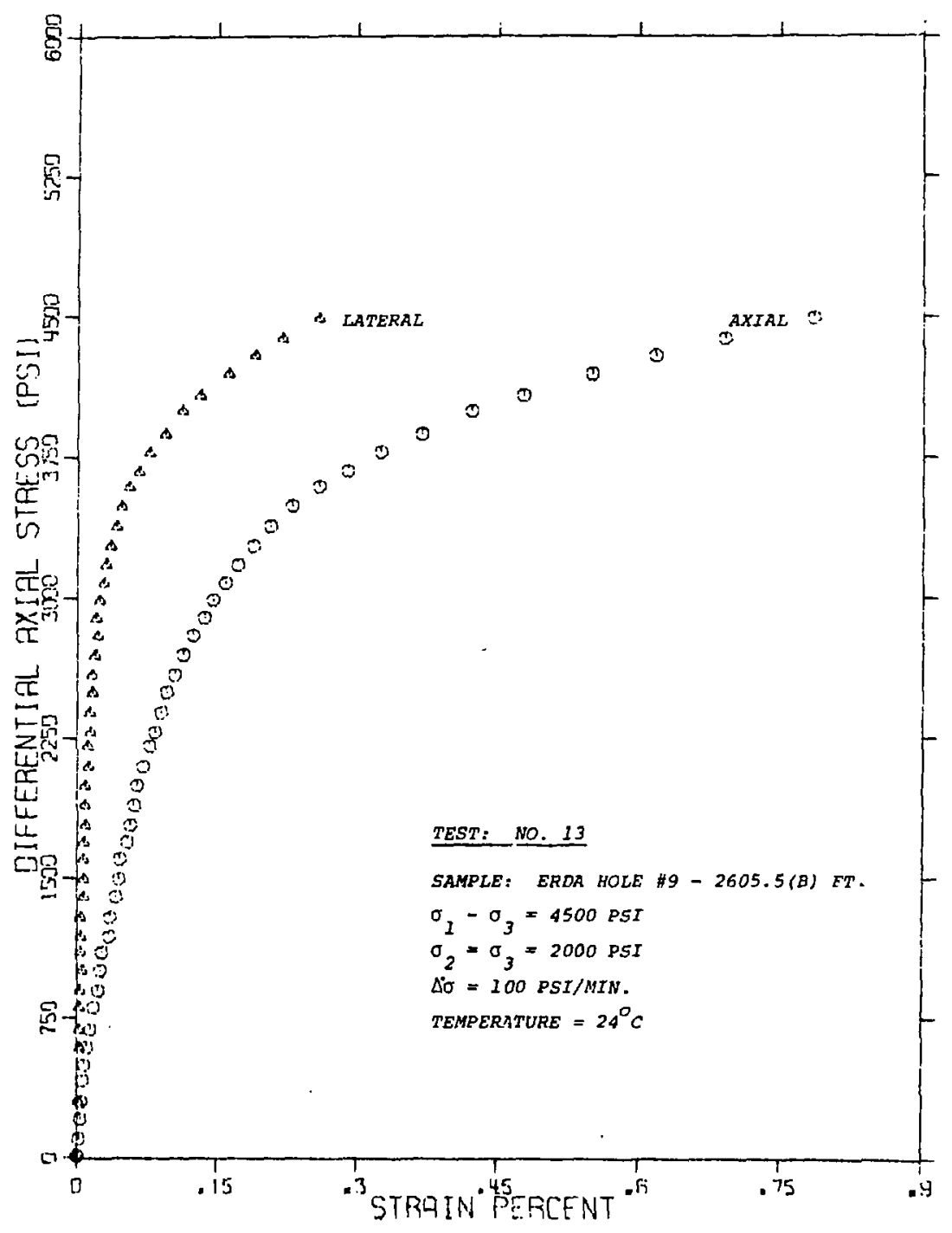

Figure A-17. Differential Axial Stress as a Function of Axial and Lateral Strain, Test 13. 


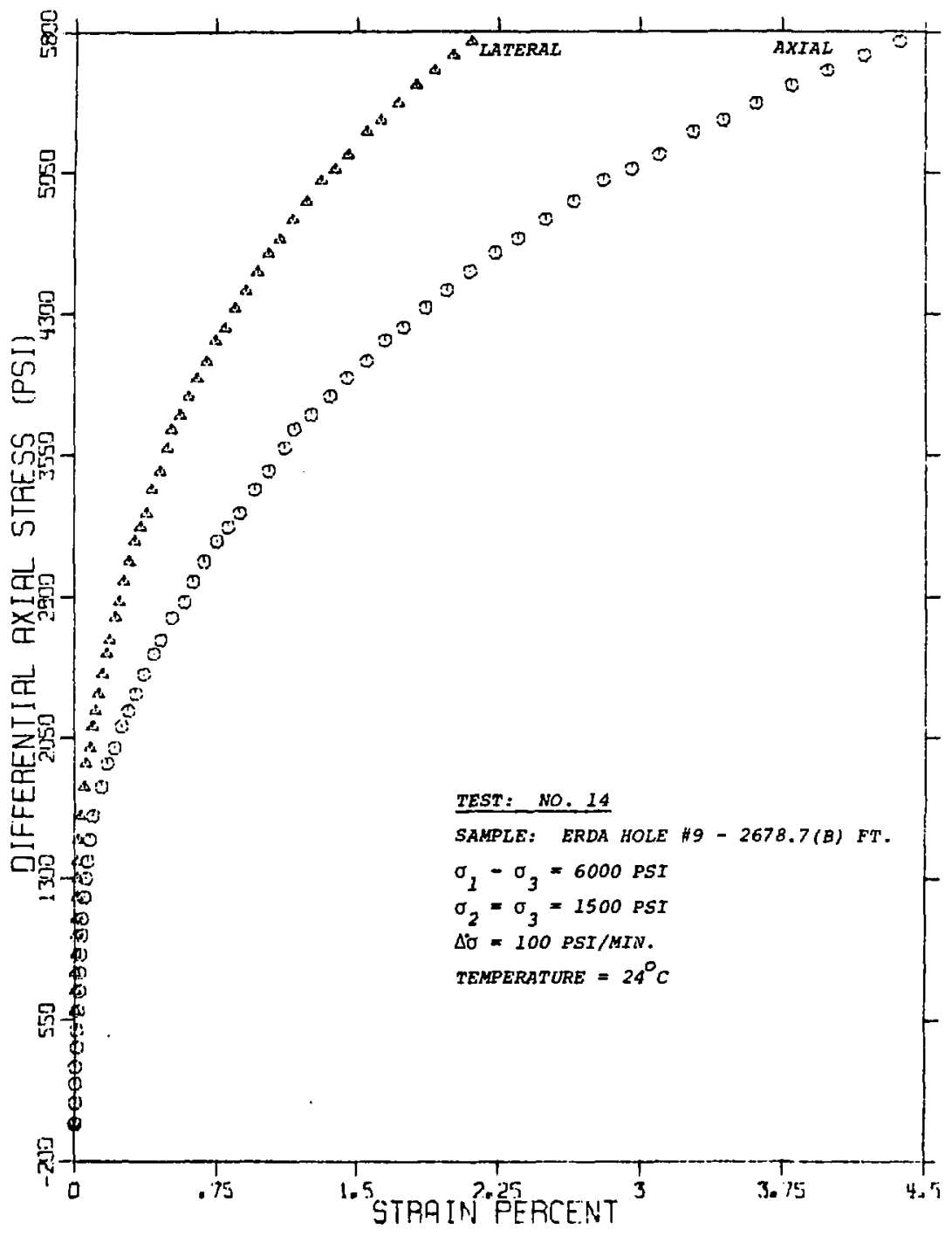

Figure A-18. Differentlal Axial Stress as a Function of Axial and Lateral strain, Test 14. 


\section{APPENDIX B}

PLOTS OF AXIAL STRAIN

AS A FUNCTION OF TIME

FIGURE NO.

B-1

B-2

B-3

B-4

B-5

B-6

B-7

B-8

B-9

E-10

$B-11$

B-12

B-13

$8-15$

$B-16$.

$8-17$

$B-18$

$B-19$

$B-20$

\section{PLOTTED PARAMETERS}

Axial strain as a Function of Time, Test 1

PAGE NO.

65

$6 \hat{0}$

67

68

69

70

71

72

73

Stage 1

Axial Strain as a Function of Time, Test 9, Stage 2

74

75

Axial Strige 1

Axial Strain as a Function of Time, Test 10, stage 2

76

77

Axial Strain as a Function of Time, Test 11, Stage 1

Stage 1

Axial Strain as a Function of Time, Test 12, Stage 2

Axial Strain as a Function of Time, Test 14

$\log _{10}$ Axial strain as a Function of $\log _{10}$ Time
(Sec.), Test 1

$\log _{10}$ Axial Strat
(Sec.), Test 2

$\log _{10}$ Axial Strain as a Function of $\log _{10}$ Time
(Sec.), Test 3 


\section{APPENDIX B (CON2" D)}

FIGURE_NO.

PAGE NO.

B-21 $\log _{10}$ Axial Strain as a Function of $\log _{10}$ Time 85 (Sec.), Test 4

$B=22$

Log 10 Axial strain as a Function of $\log _{10}$ Time
(Sec.). Test 5

$\log _{30}$ Axial strain as a Function of $\log _{10}$ Time

$\log _{10}$ Axial strain as a Function of $\log _{10}$ Time
(Sec.), Test 7

$\log _{10}$ Axial strain as a Function of Log 10 rime (sec.), Test 8

$\log _{10}$ Axial strain as a Function of $\log _{10}$ Time
(sec.), Test 9, stage 1

Log 10 Axial strain as a Function of $\log _{10}$ Time
(Sec.), Test 10, Stage 1 


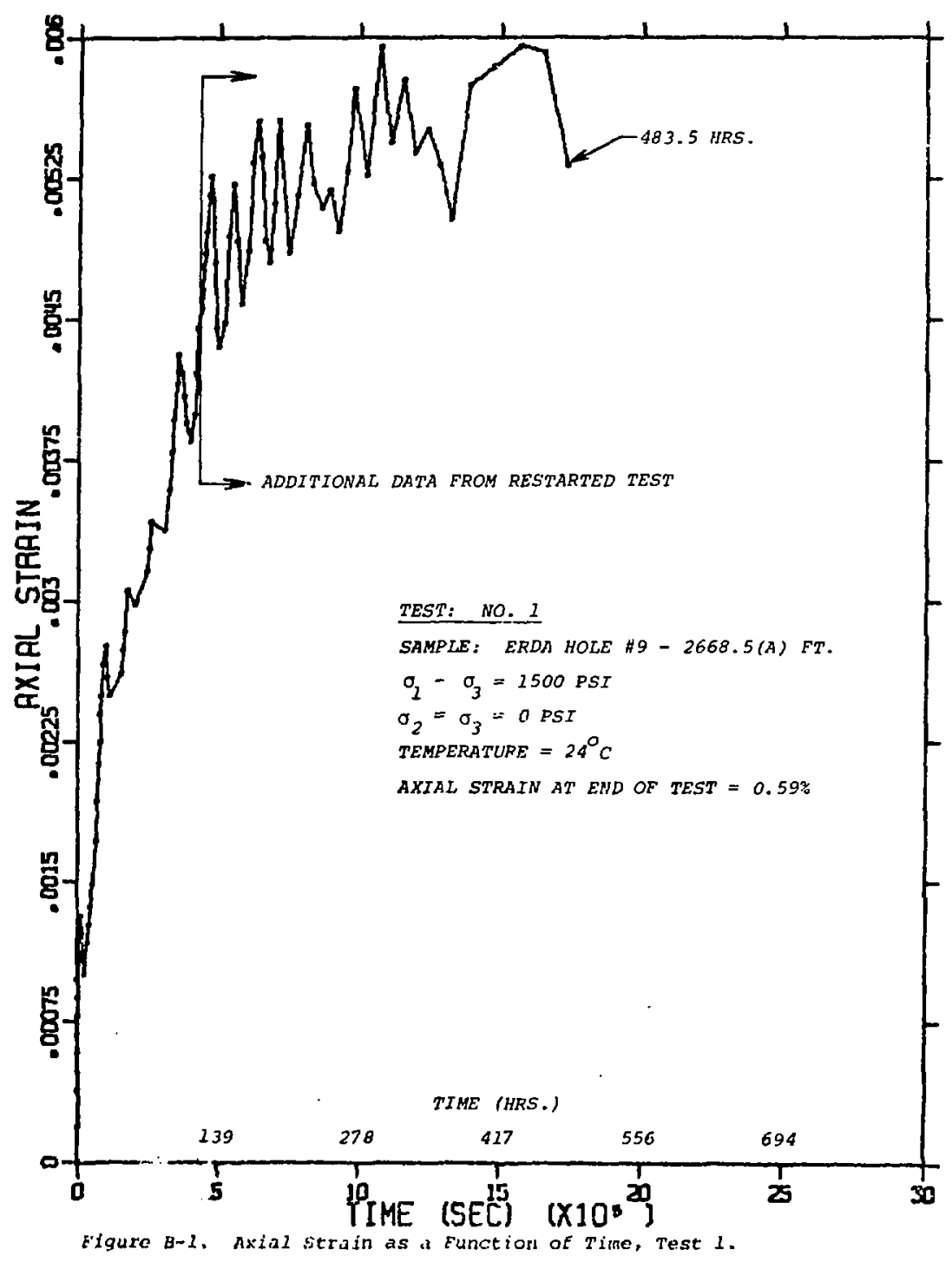




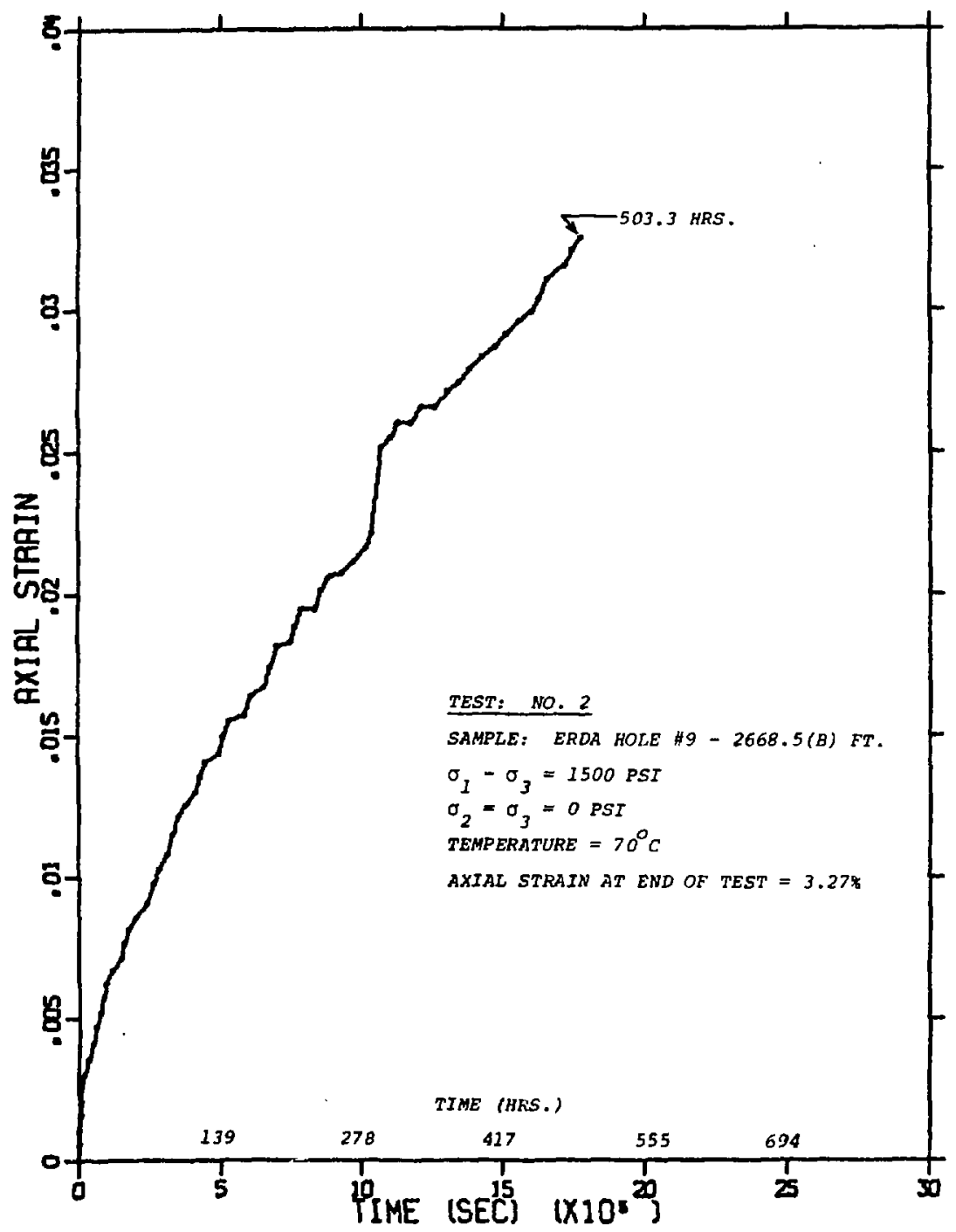

Figure B-2. Axial strain as a Function of Time, Test 2. 


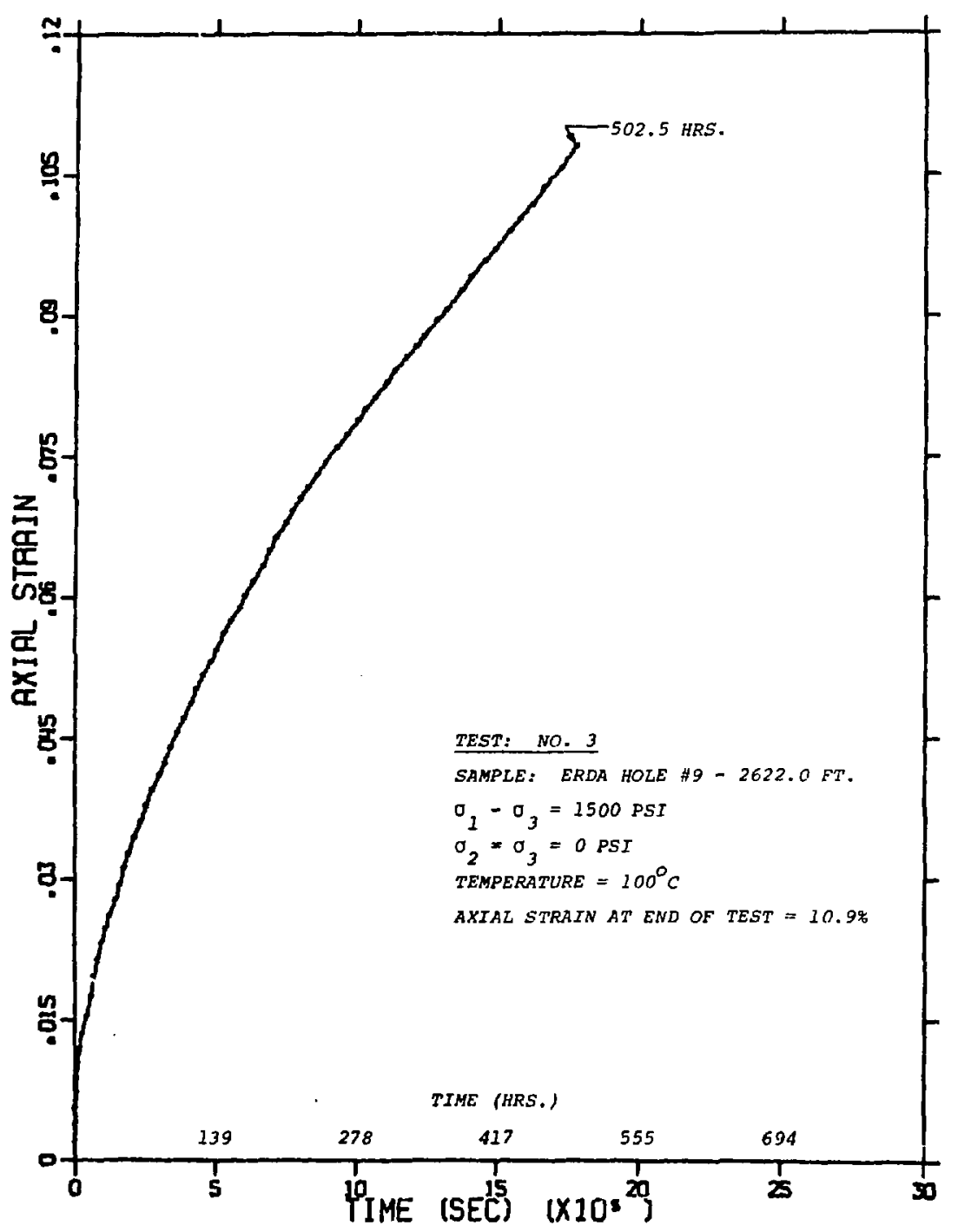

Flqure B-3. Axial strain as a Function of Time, Test 3. 


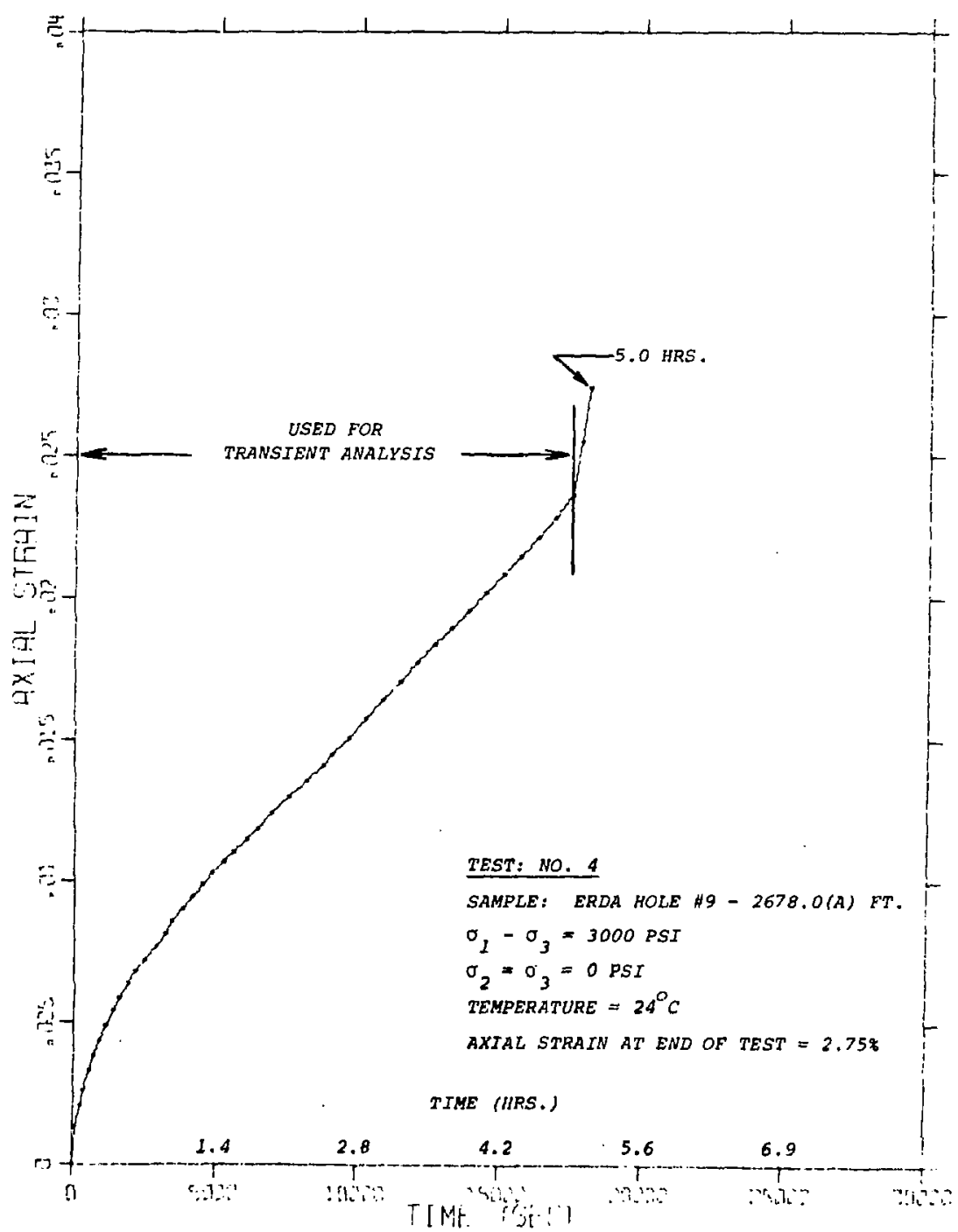

Flgure B-4. Axial strain as a Function of time, Test 4. 


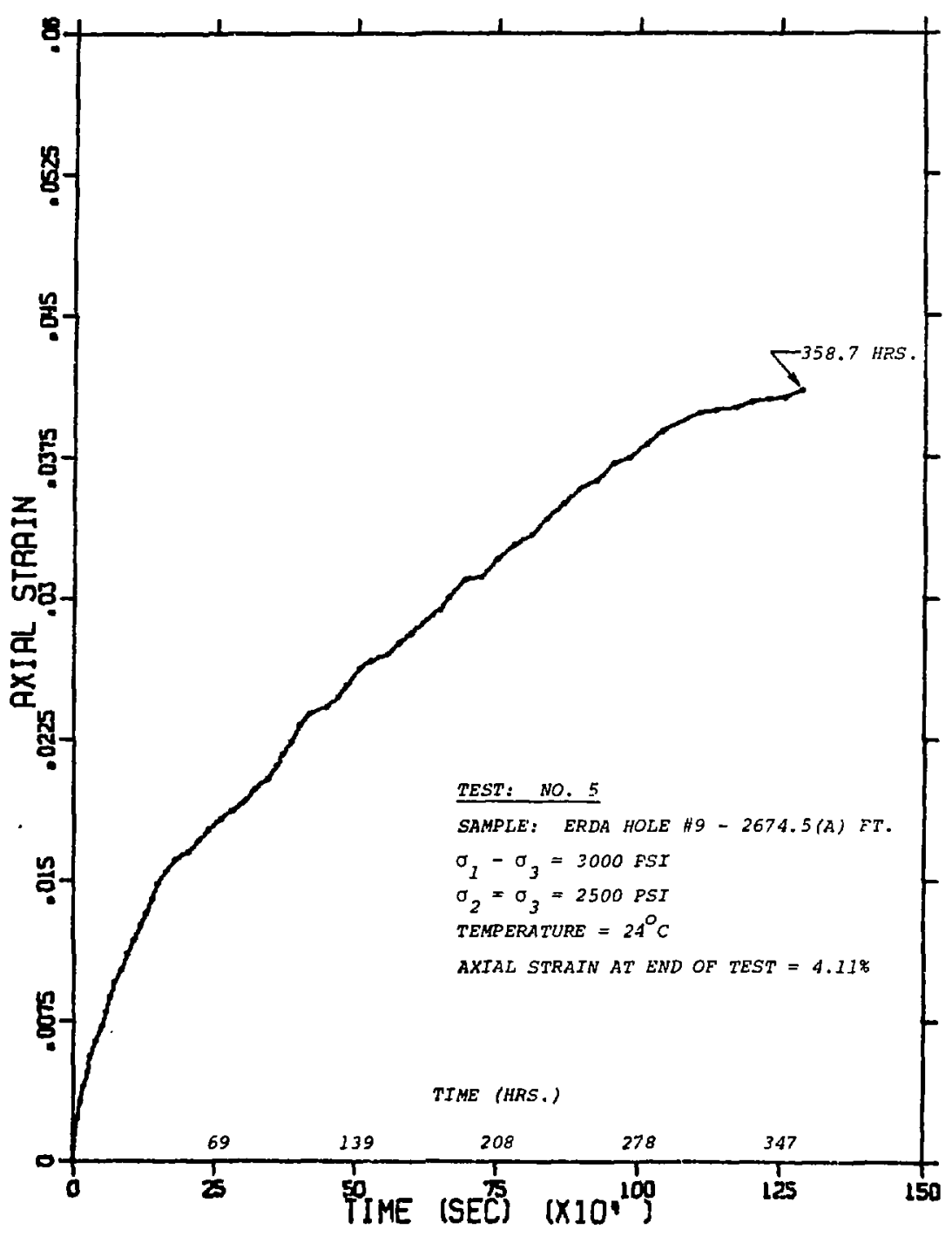

Flgure B-5. Axial straln as a Function of Time, rest 5. 


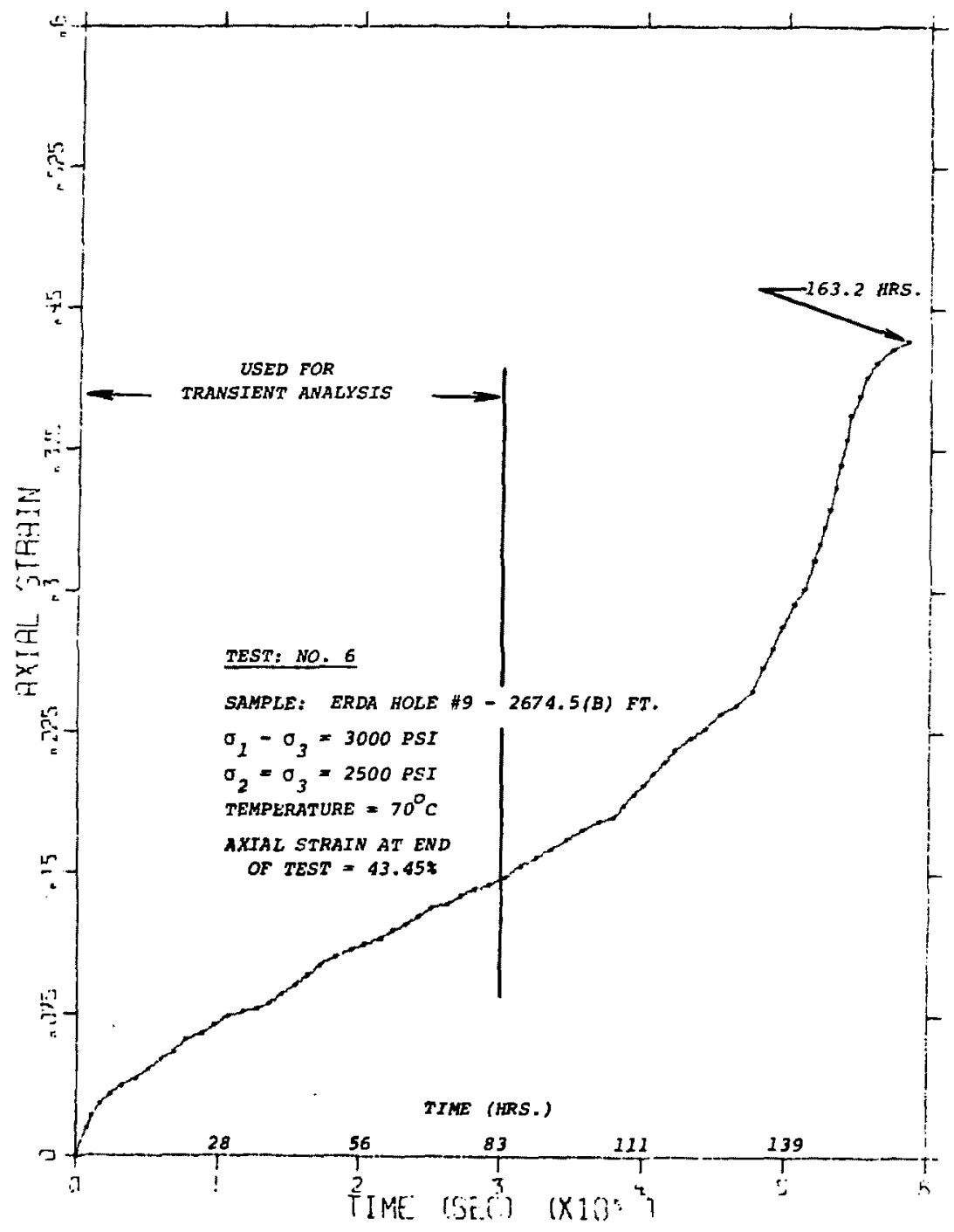

Flgure 8-6. Axial Strain as a Function of Time, Test 6 . 


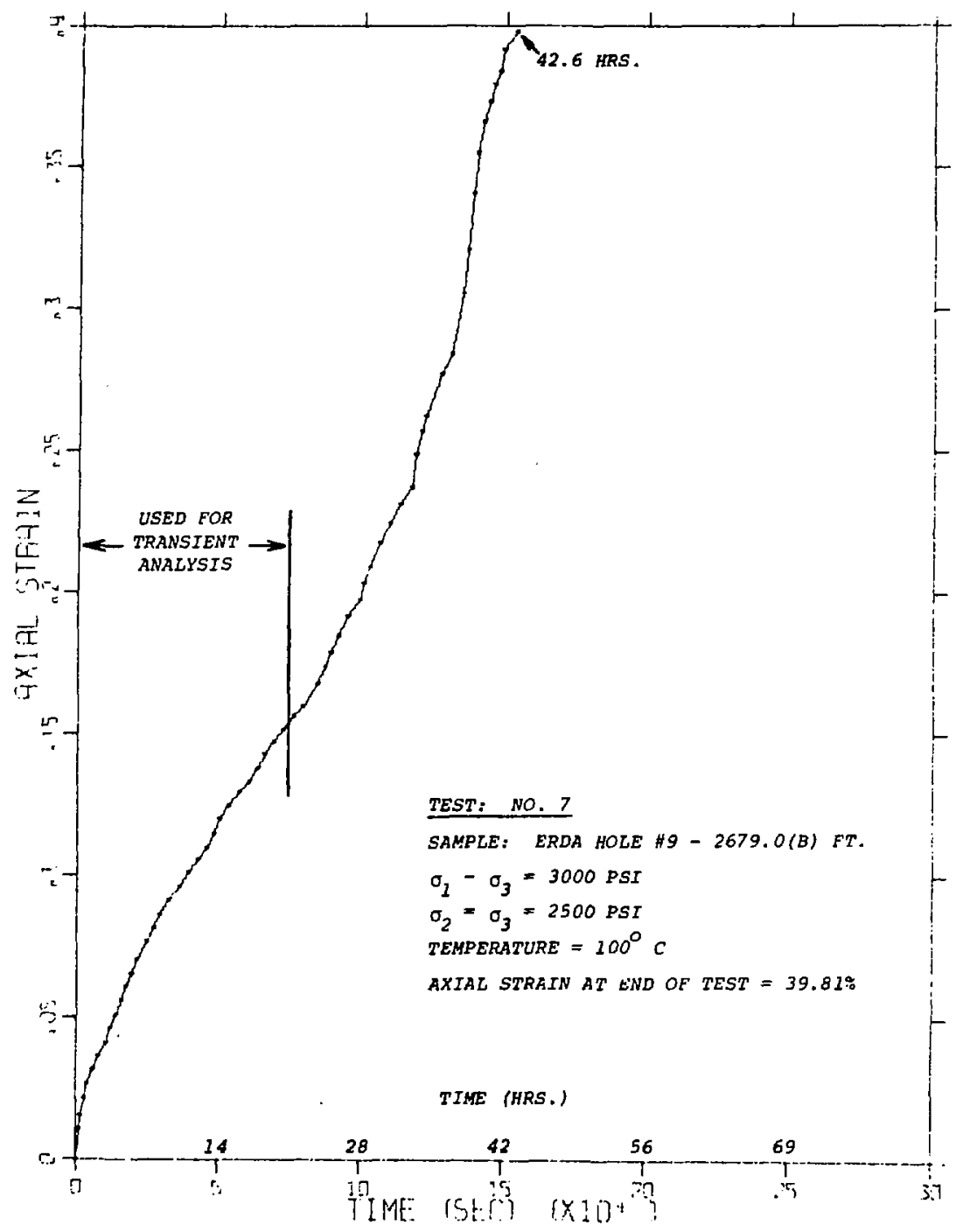

Figure B-7. Axial Strain as a function of Time, Test 7 . 


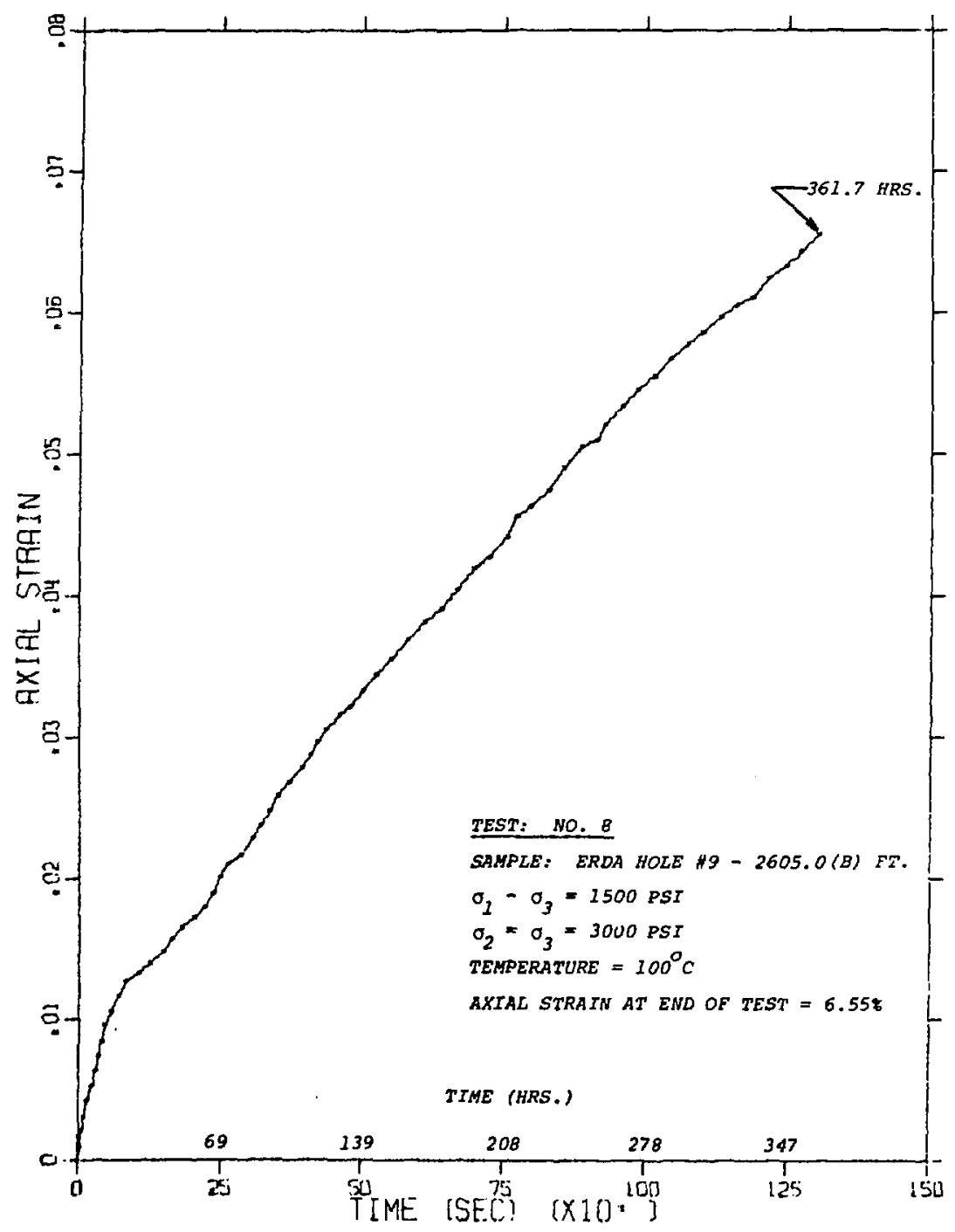

Figure B-8, Axial strain as a Function of Time, Test 8 . 


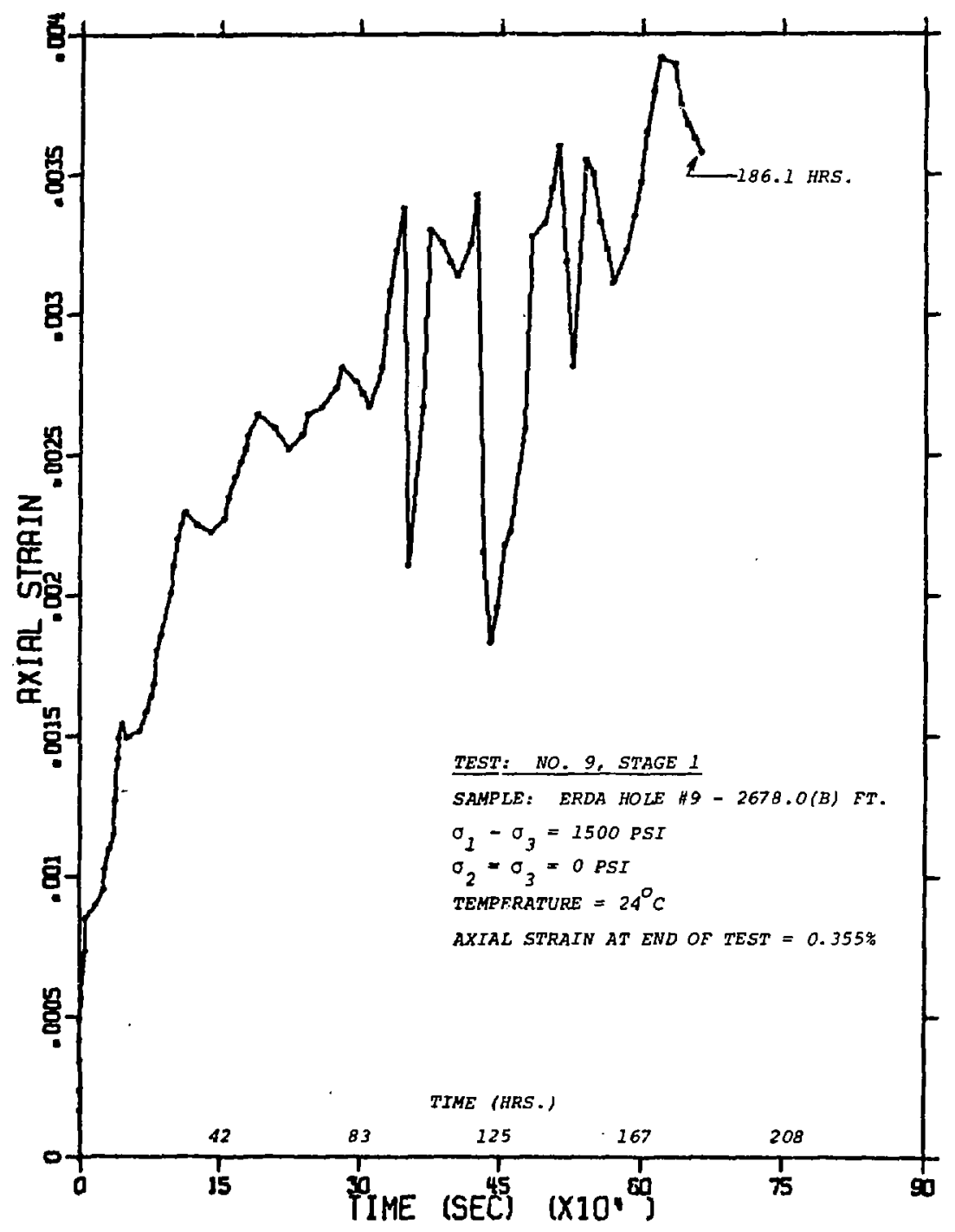

Figure B-9. Axial strain as a function of Time, Test 9, stage 1. 


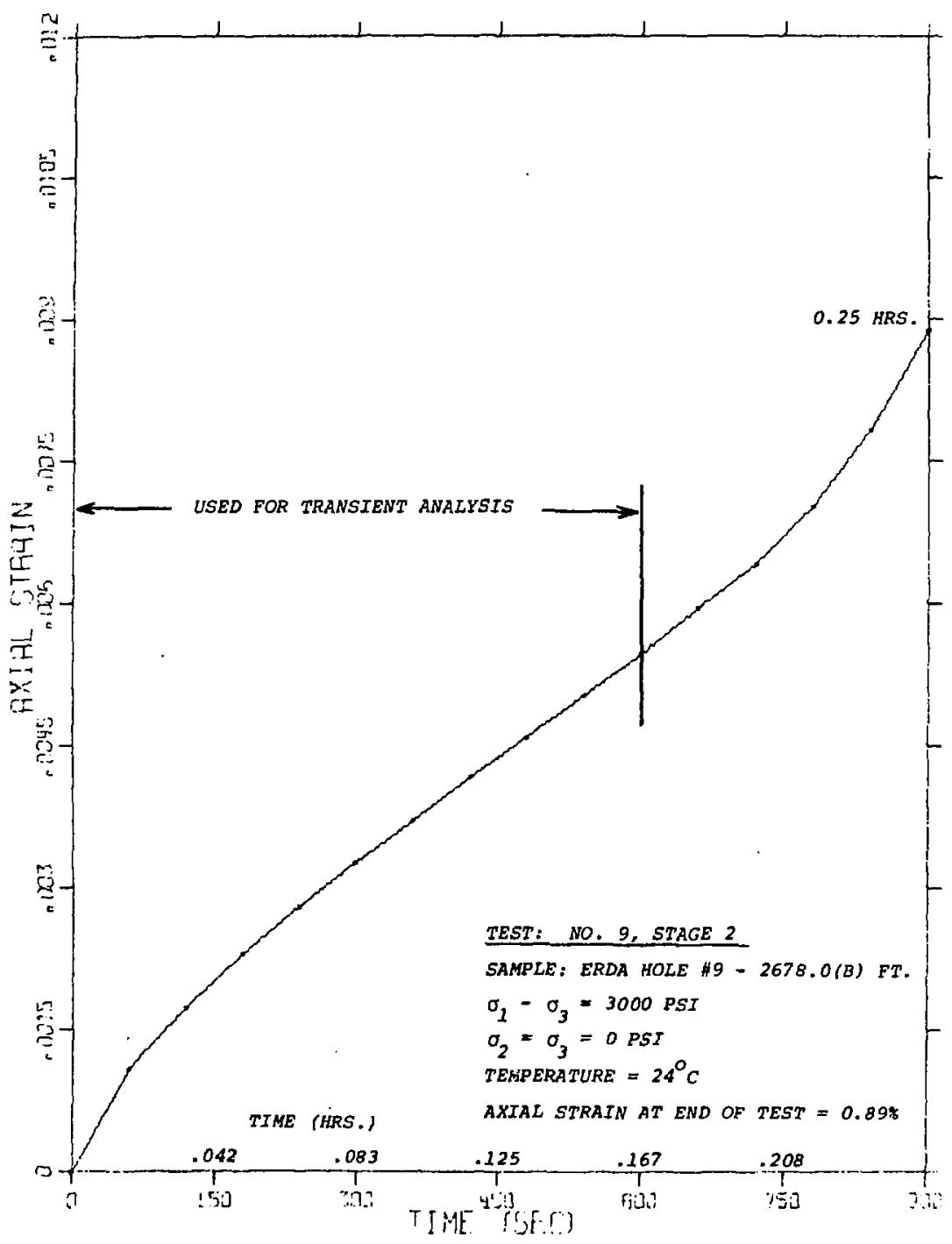

Figure B-30. Axial Strain as a function of rime, Test 9, stage 2. 


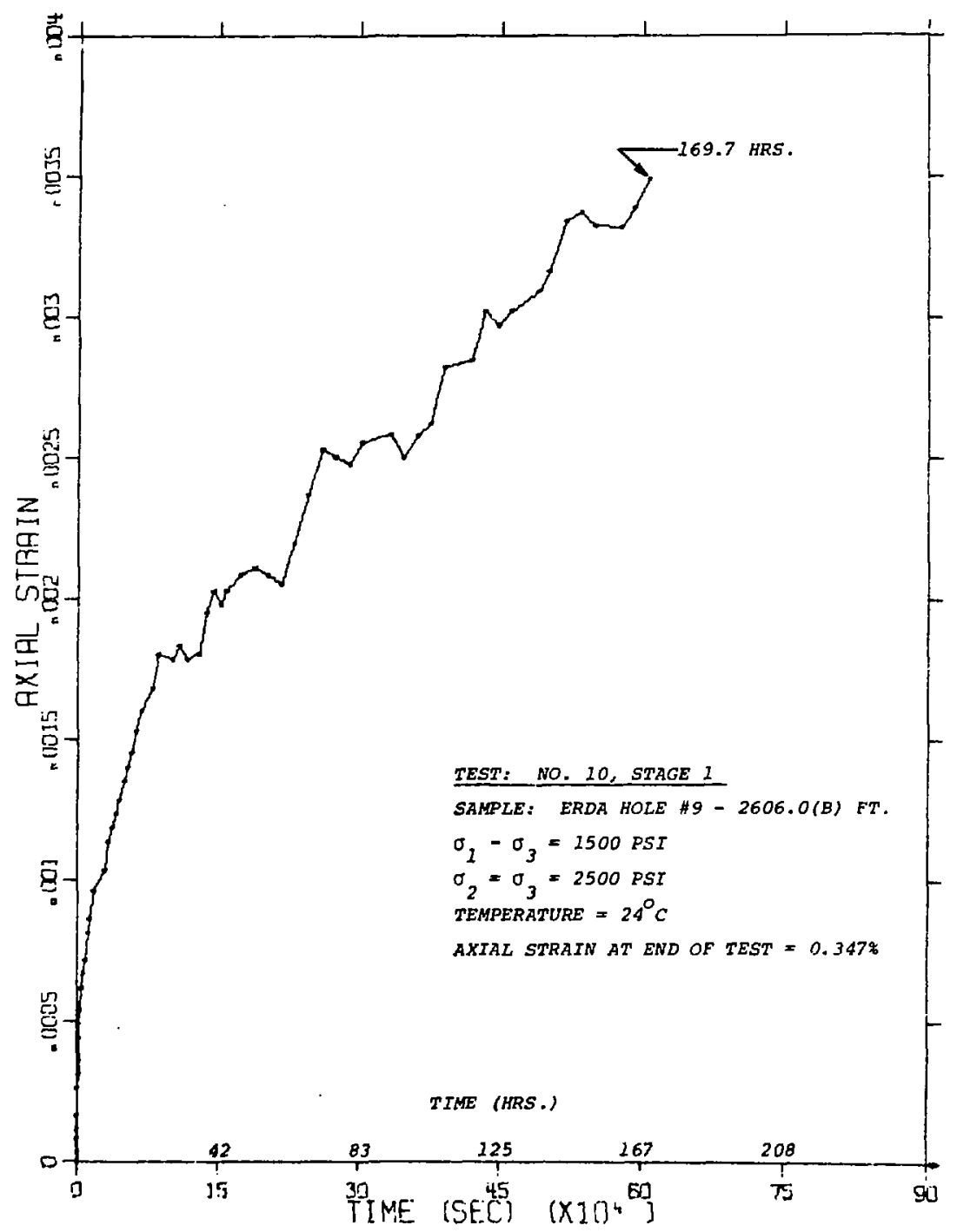

Figure B-11. Axial sirain as a Function of Time, Test 10, stage 1. 


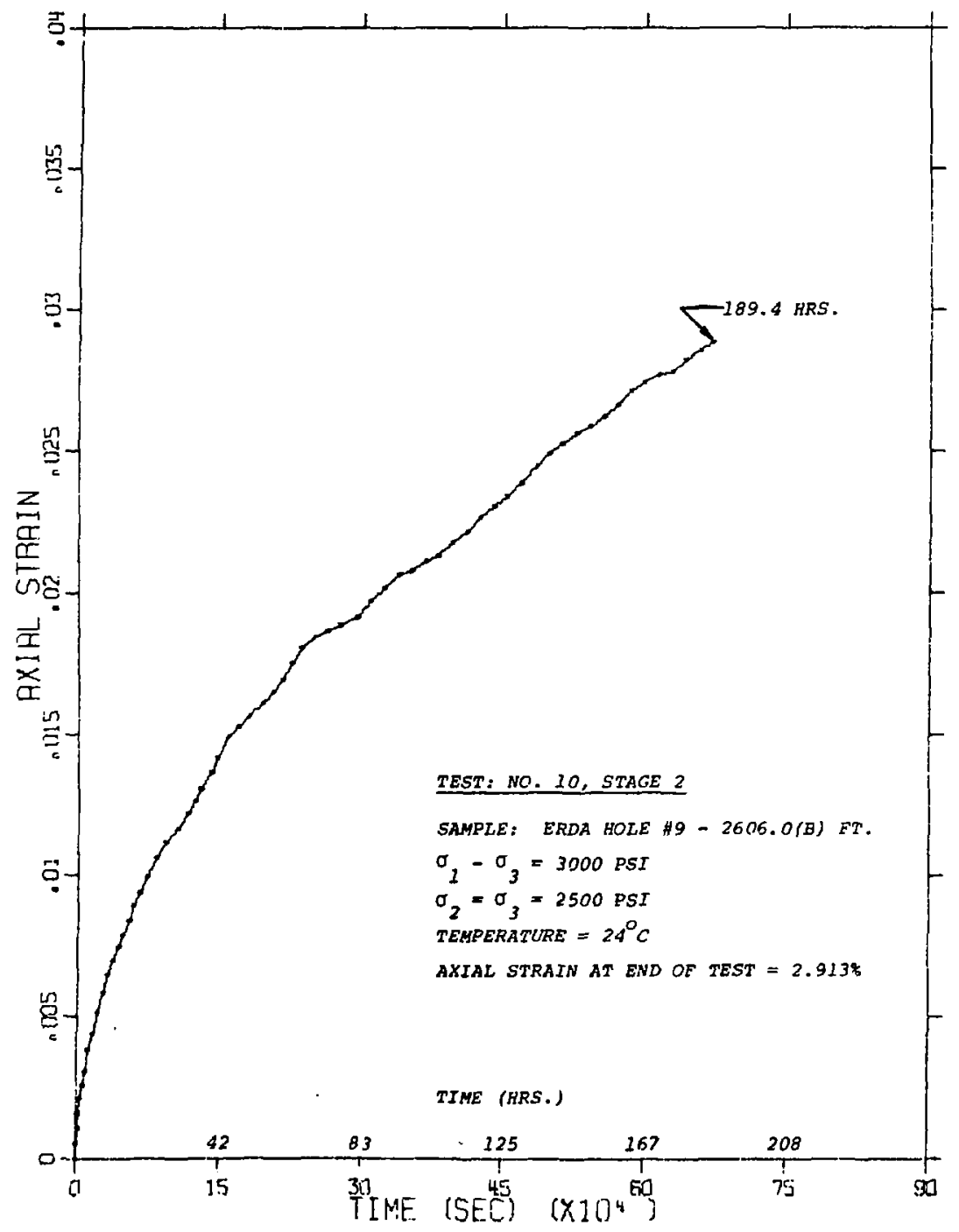

Figure B-12. Axial strain as a runction of time, Test 10, stage 2. 


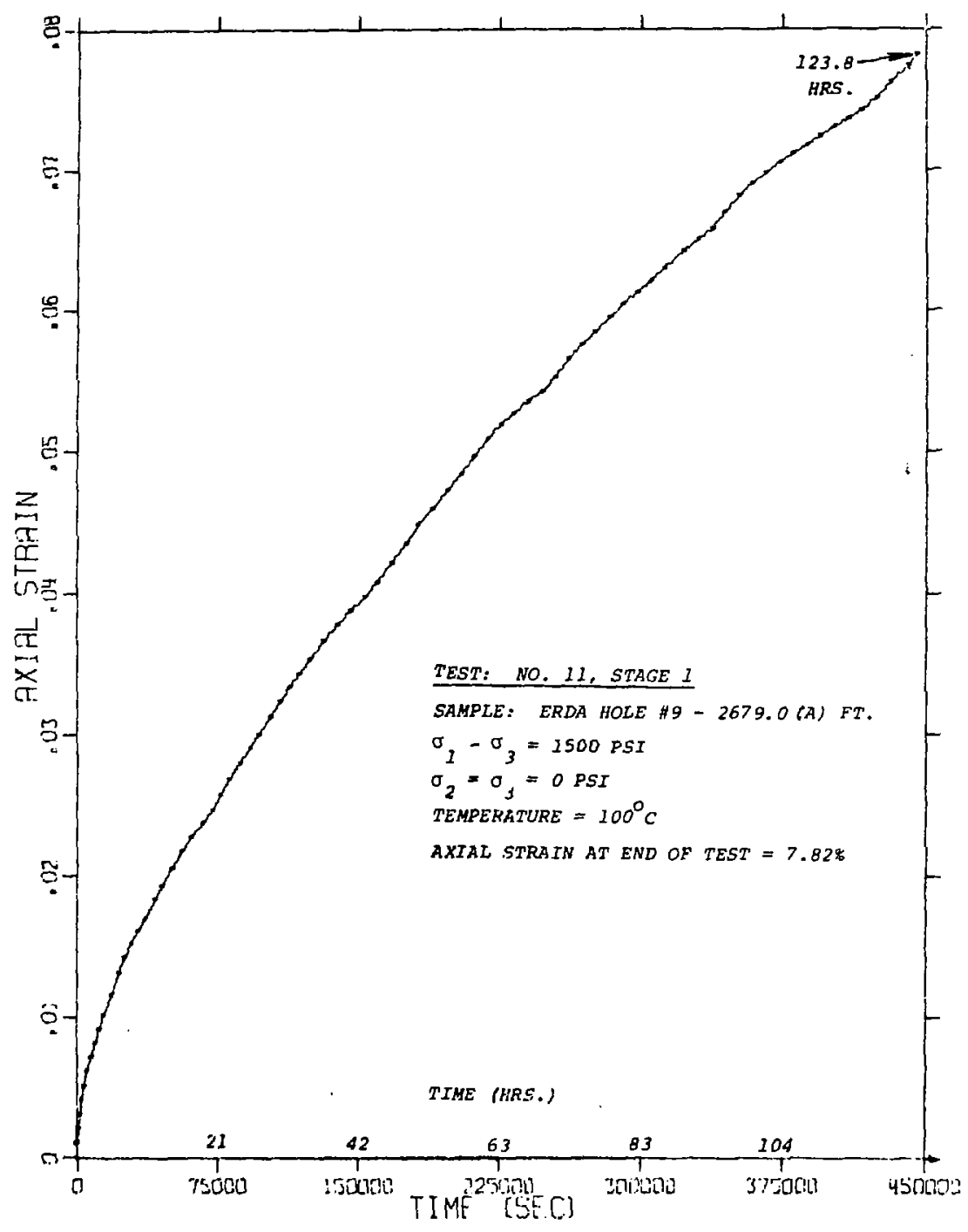

Figure B-13. Axial stratn as a Function of Time, Test 11, stage 1. 


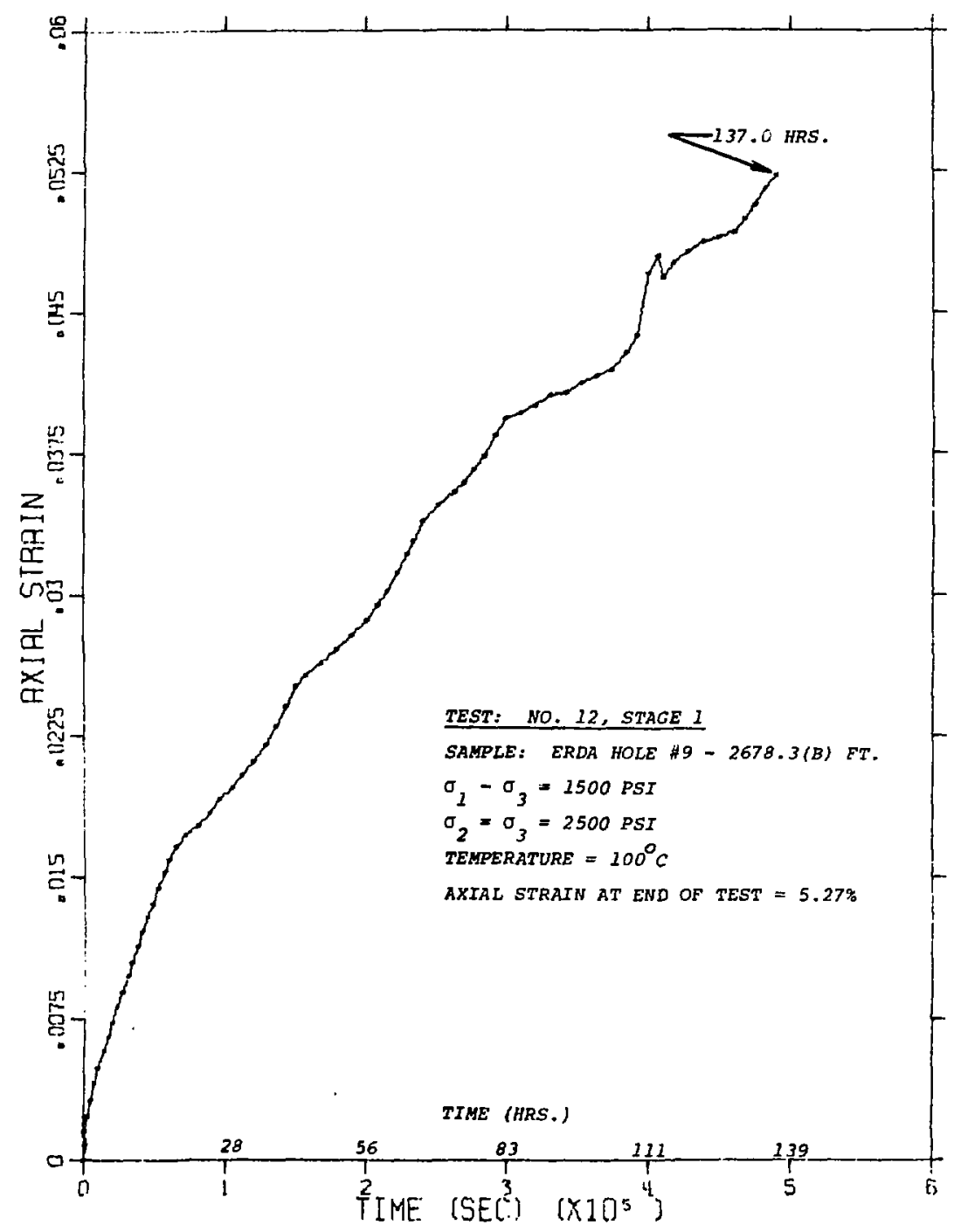

Figure B-14. Axial Strain as a Function of Time, Test 12, stage 1. 


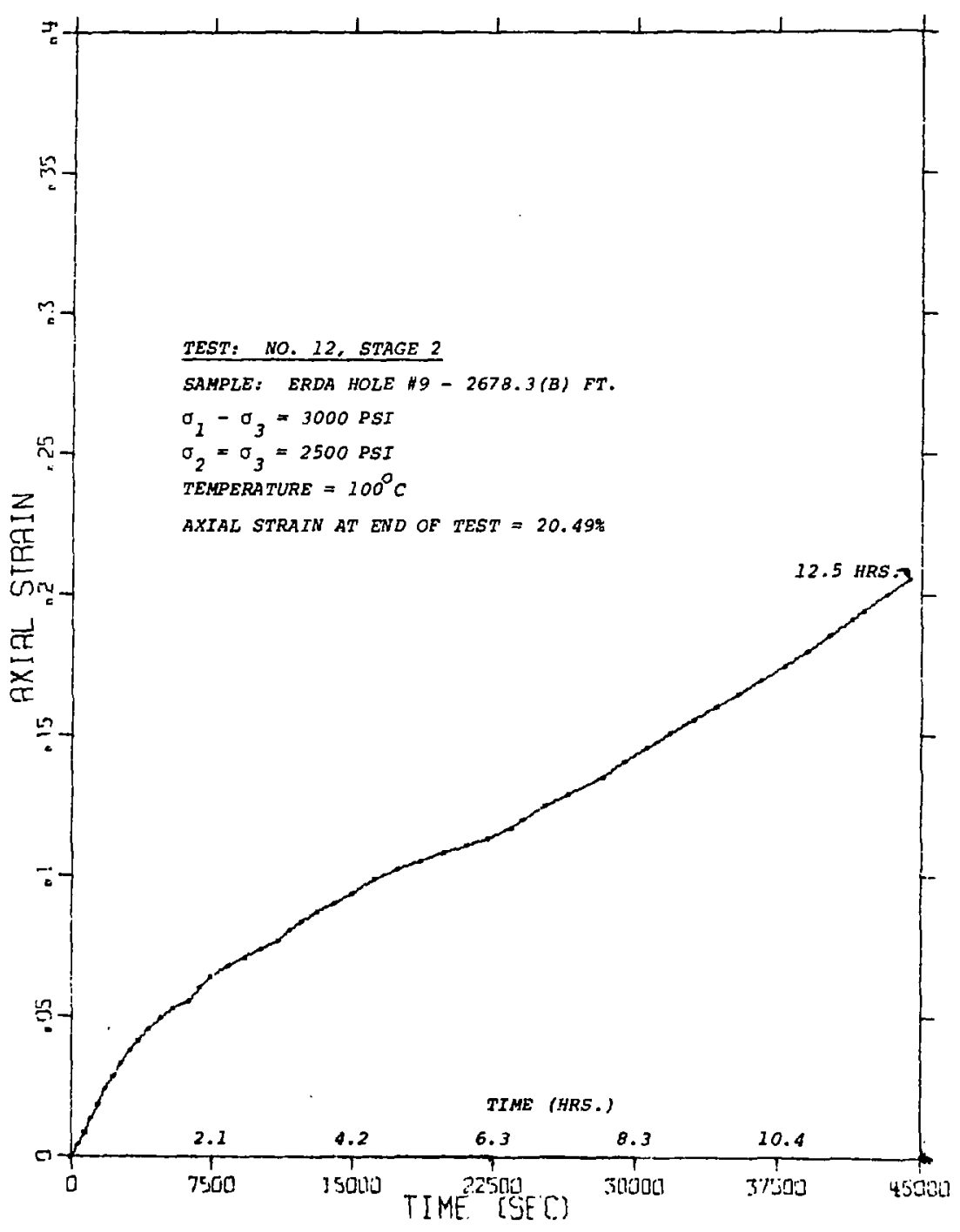

Figure b-15. Axial strain as a Function of Time, Test 12, stage 2. 


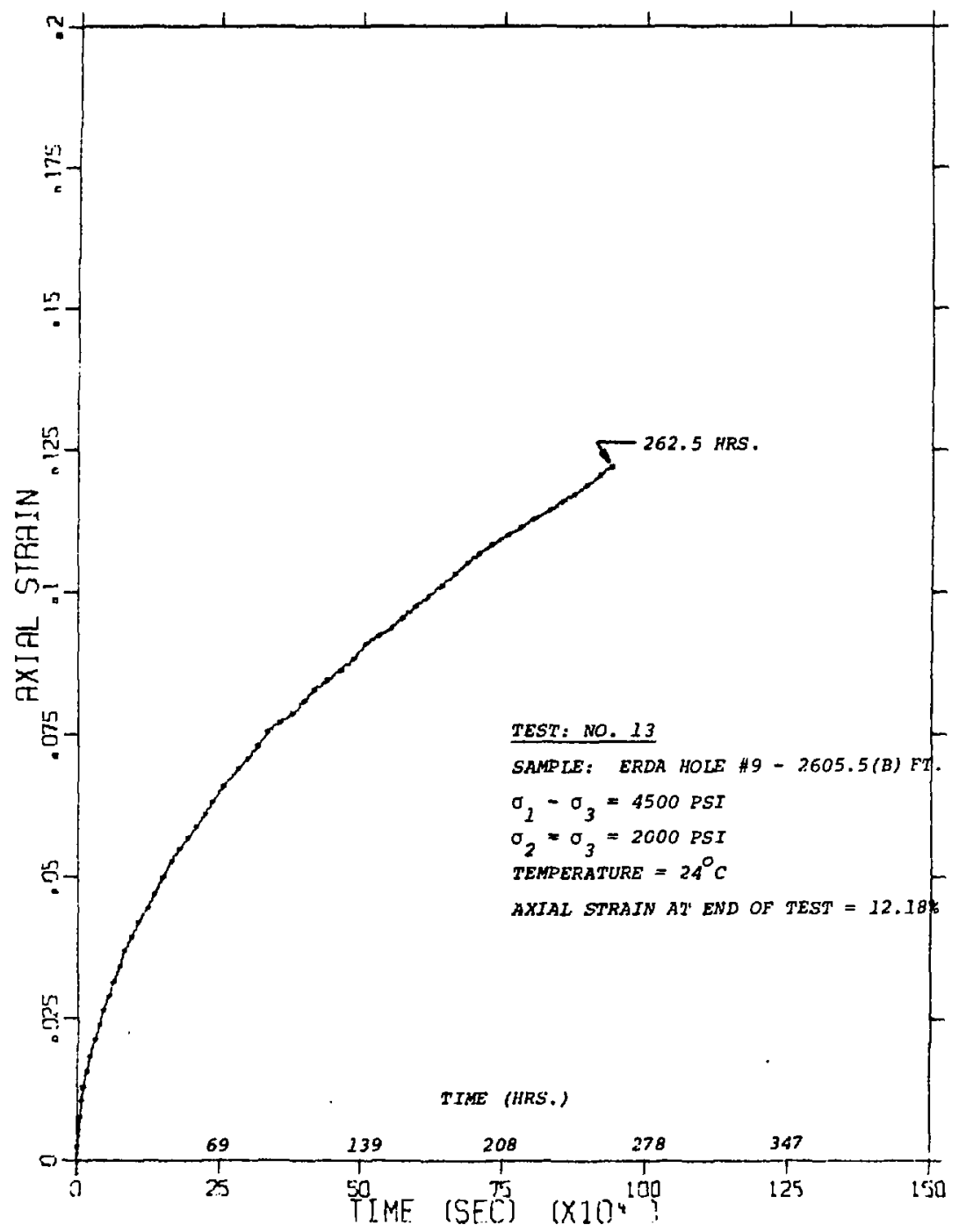

Figure B-16. AxiaI Strain as a Function of Time, Test 13. 


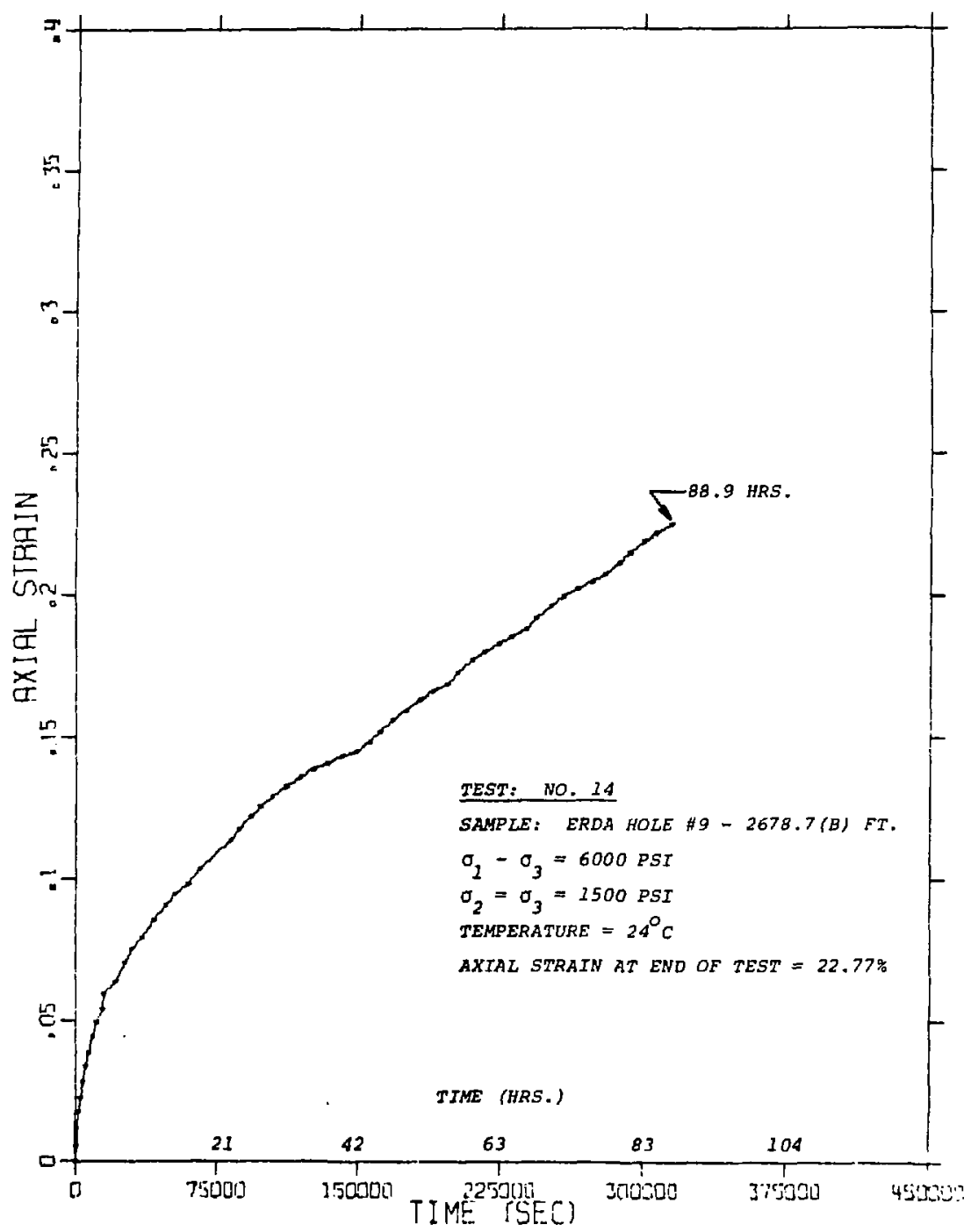

Figure B-17. Axial strain as a Function of Time, Test 14. 


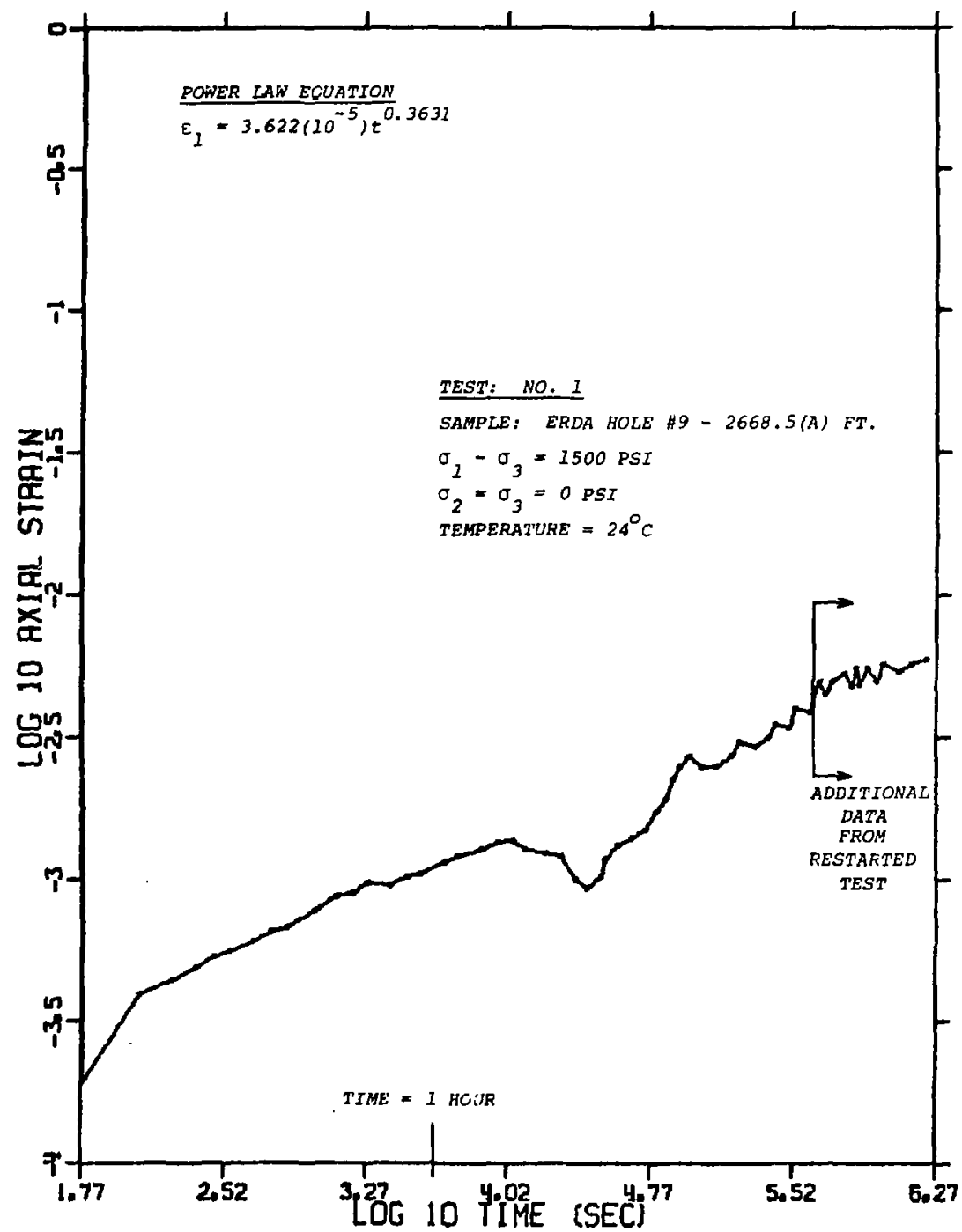

rigure $A-18 . \log _{10}$ Axial strain as a Function of Log 10 Time (Sec.). Test 1 . 


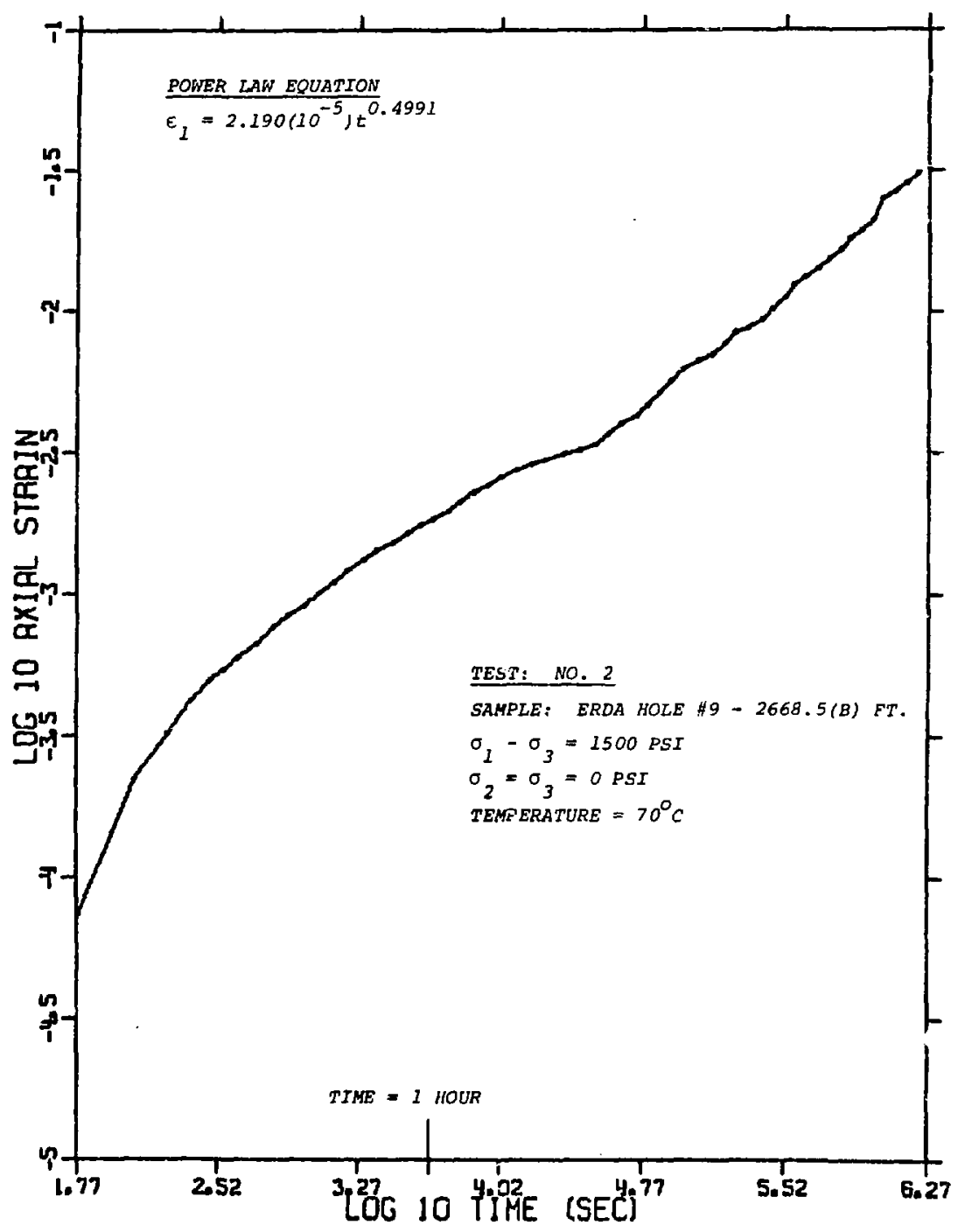

Figure 8-19. $\log _{10}$ Axial strain as a Function of $\log _{10}$ Time (Sec.), Test $\therefore$. 


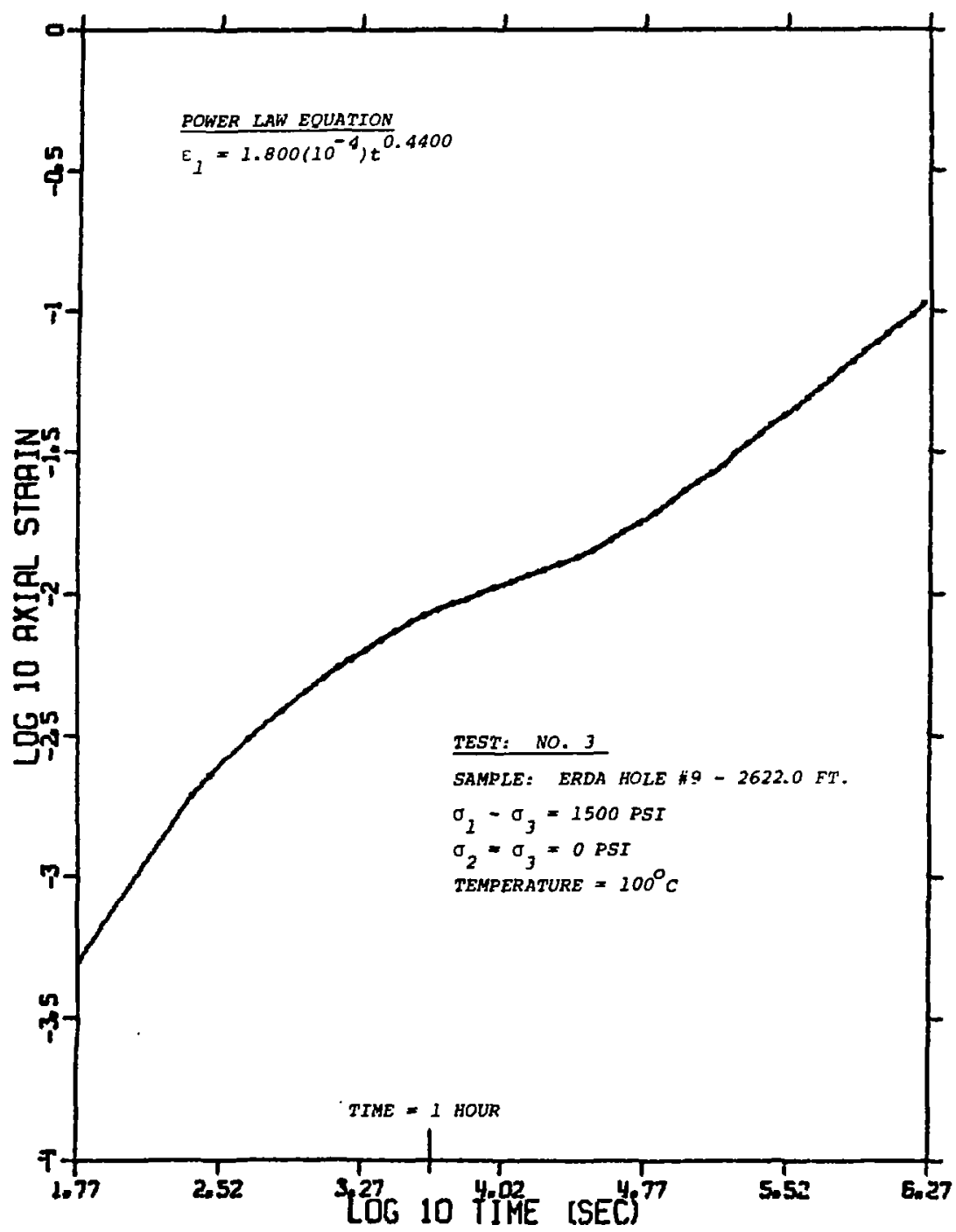

Figure $8-20 . \quad \log _{10}$ Axial strain as a Function of $\log _{10}$ time (Sec.), Test 3. 


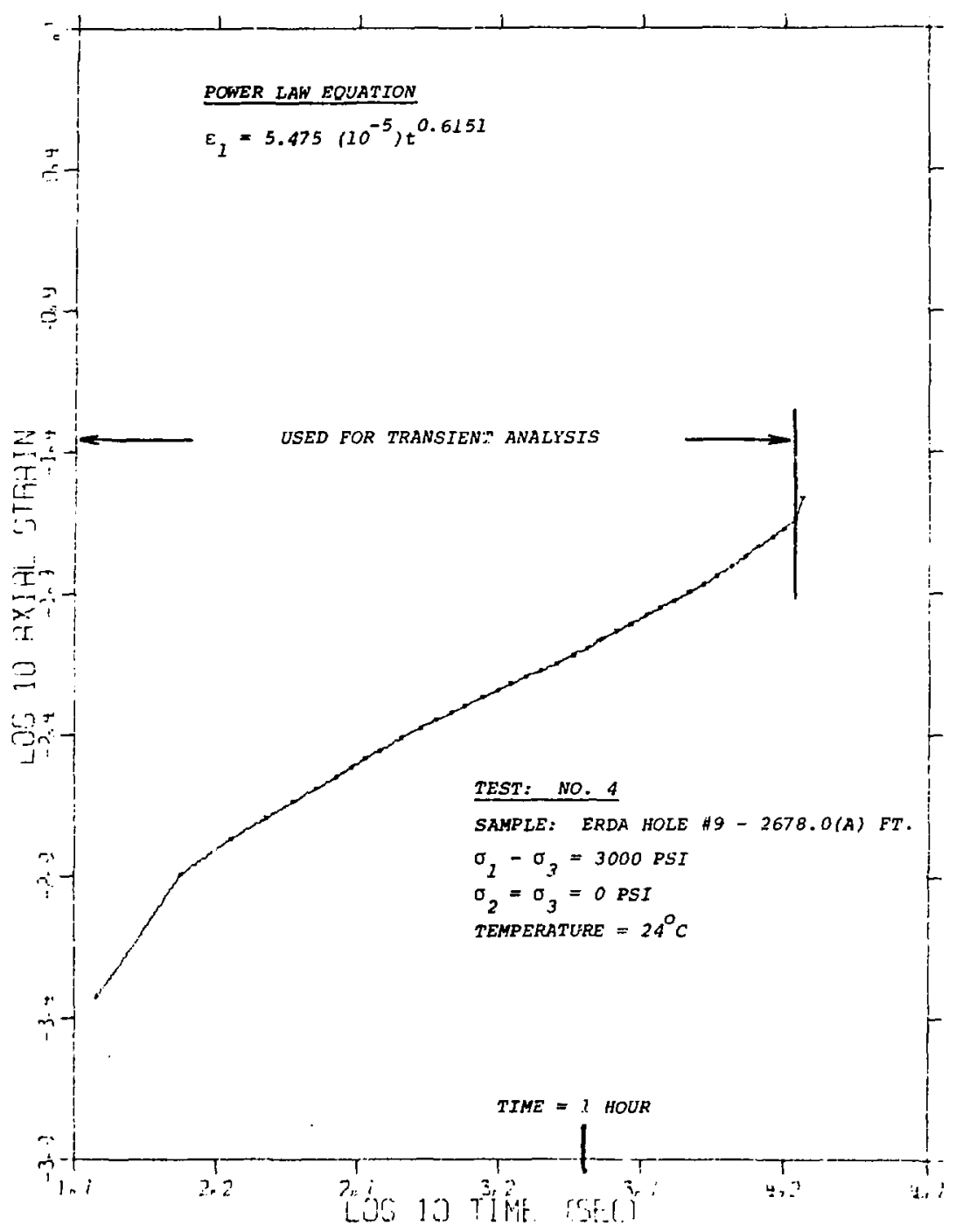

Figure B-22. Lay ${ }_{10}$ Axial strain as a Function of $\log _{10}$ Time (Sec).. Test 4 . 


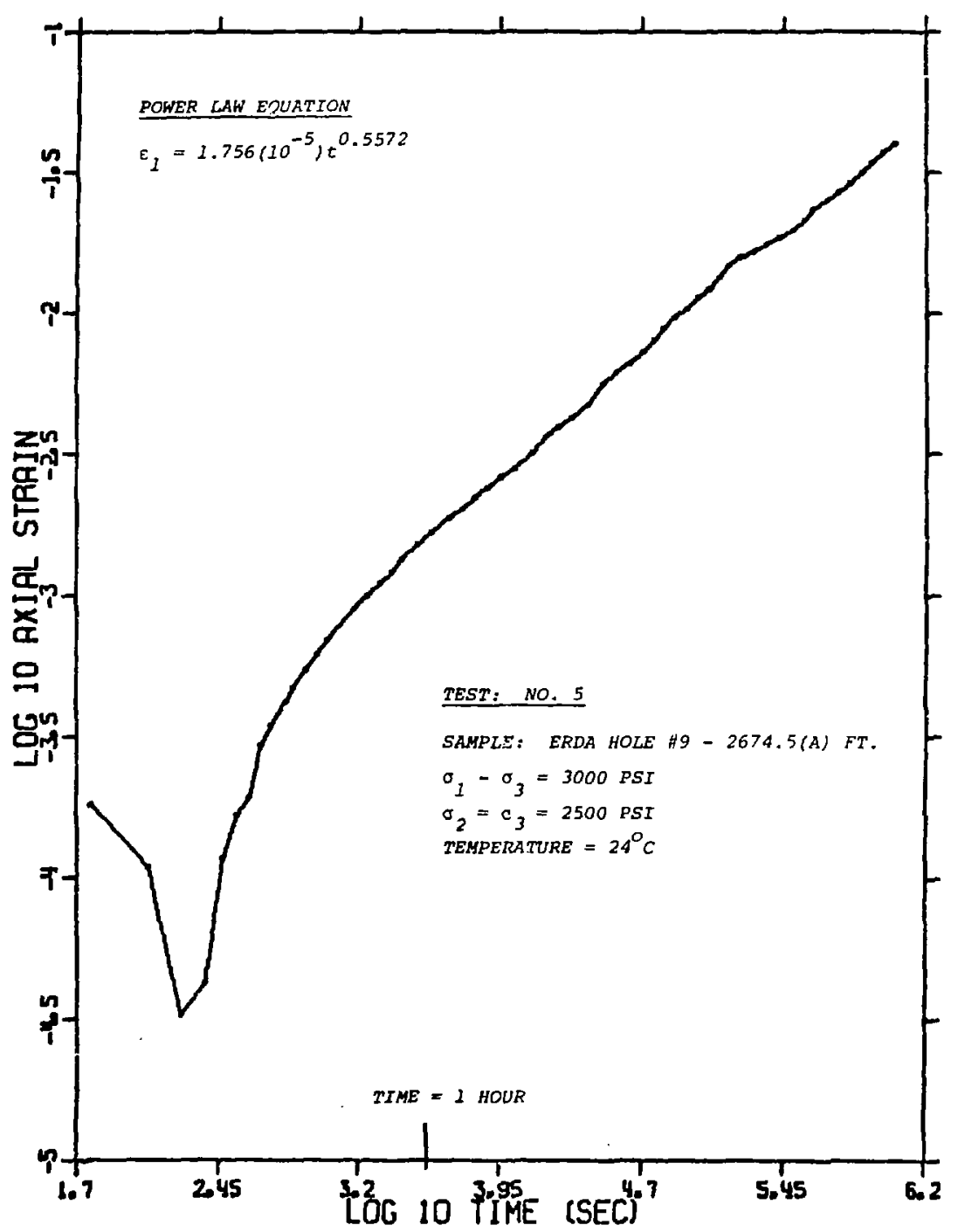

Figure 8-?2. $\log _{10}$ Axial Strain as a function of $\log _{10}$ Time (Sec.), Test 5.

86 


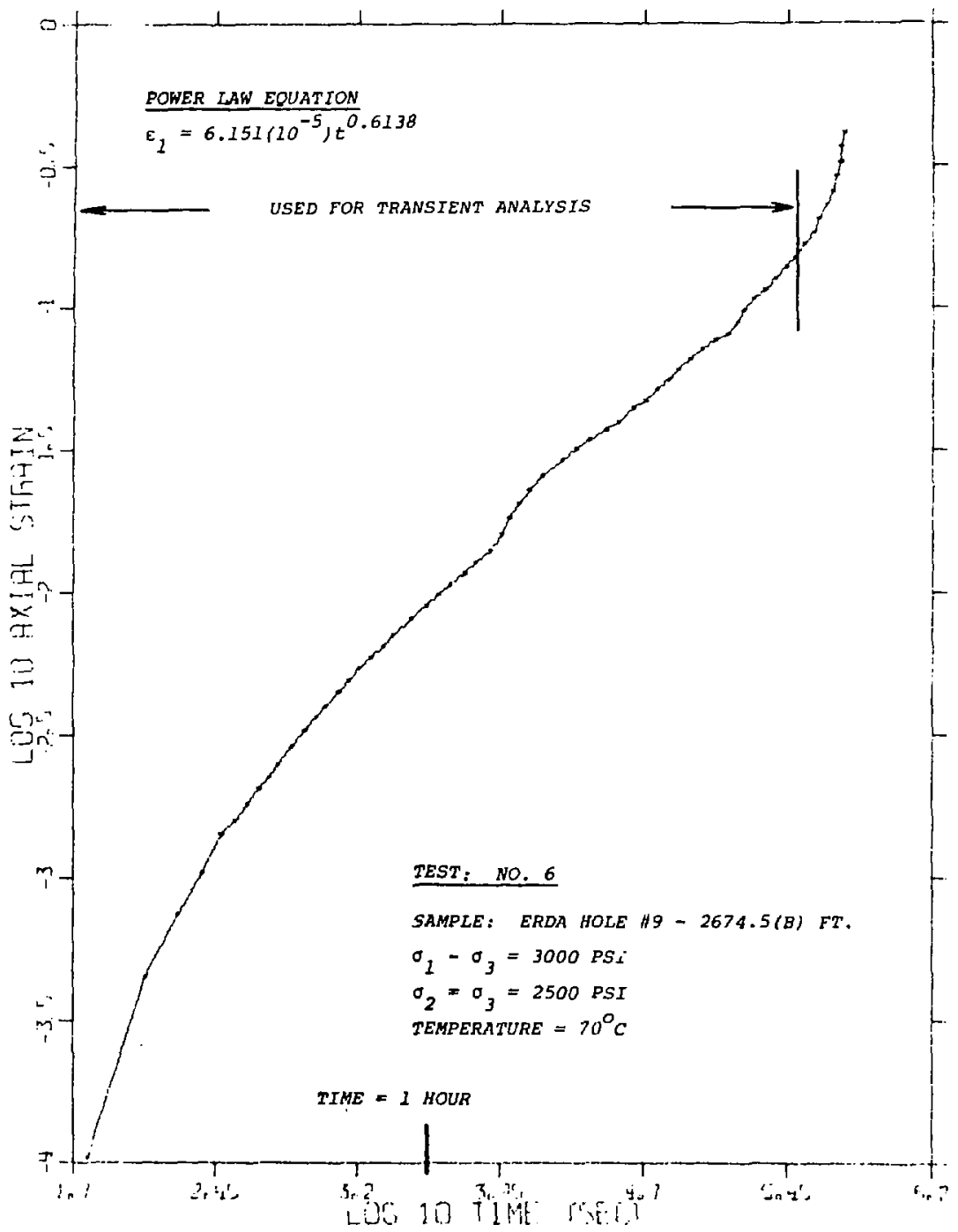

Figure B-23. $\log _{10}$ Axial strain as a Function of $\log _{10}$ Time (Sec.). Test "

87 


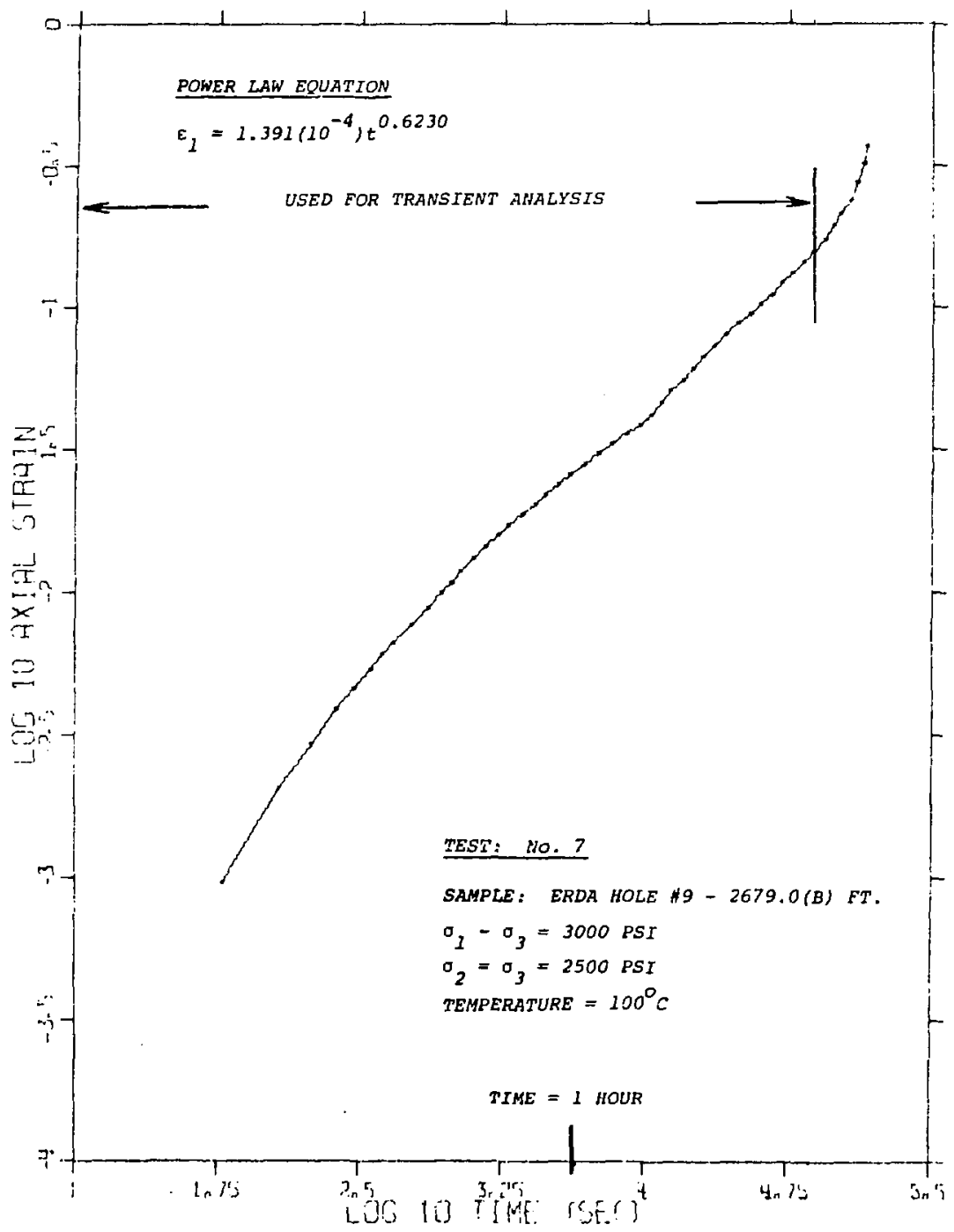

Figure $8-24$. $\log _{10}$ Axial strain as a Function of $\log _{10}$ time (Sec.), Test 7 


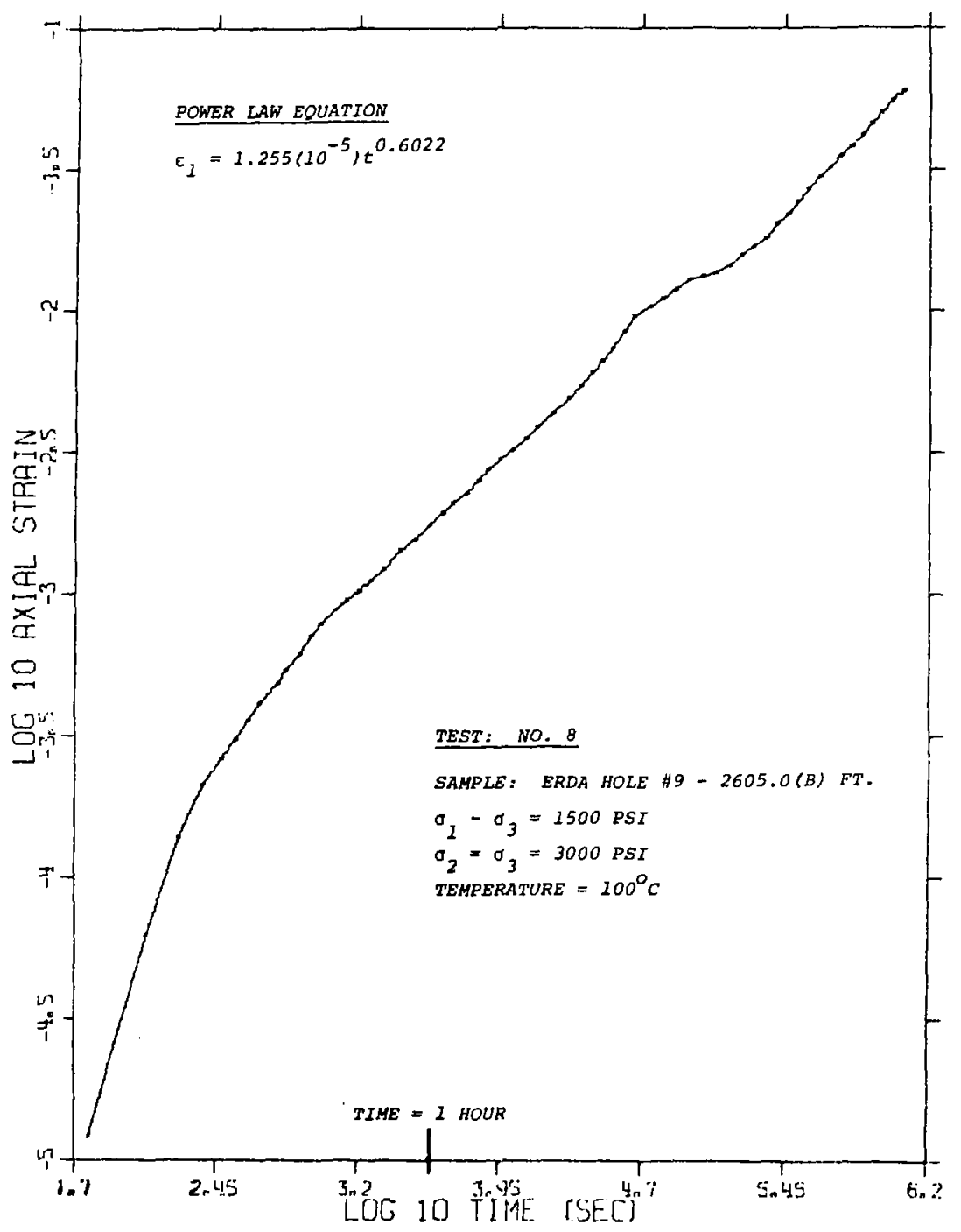

Eigure B-25. $\log _{10}$ Axial strain as a Function of $\log _{10}$ Time (sec.), Tost 8 . 


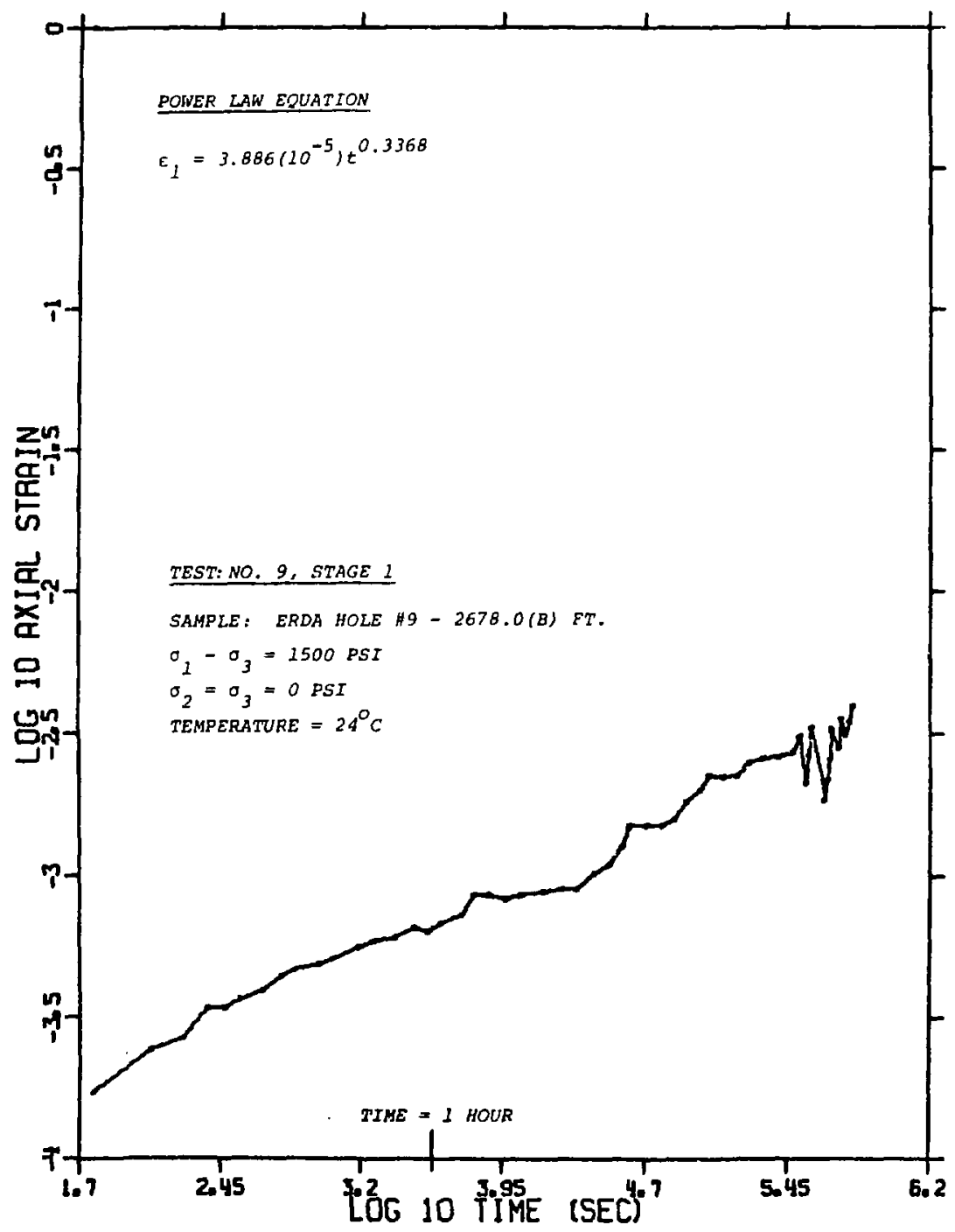

Flgure $8-26 . \log _{10}$ Axial strain as a Function of $\log _{10}$ Time (Soc.), Test 9, Stage 1 . 
POWER LAW EUUATIOA:

Rupture - No Equation Fitted

$\vdots$
$\because$
$\because$
$\because$
$\vdots$
$\because$
$\because$
$\because$
$\because \vdots-$

TEST: NO. 9, STAGE 2

SAMPLE: ERDA HOLE H9-2678.O(B) FT.

$\sigma_{1}-\sigma_{3}=3000$ PSI

$\sigma_{2}=\sigma_{3}=0$ PSI

TEMPERATURE

$\because$ USED FOR TRANSIENT ANALYSIS

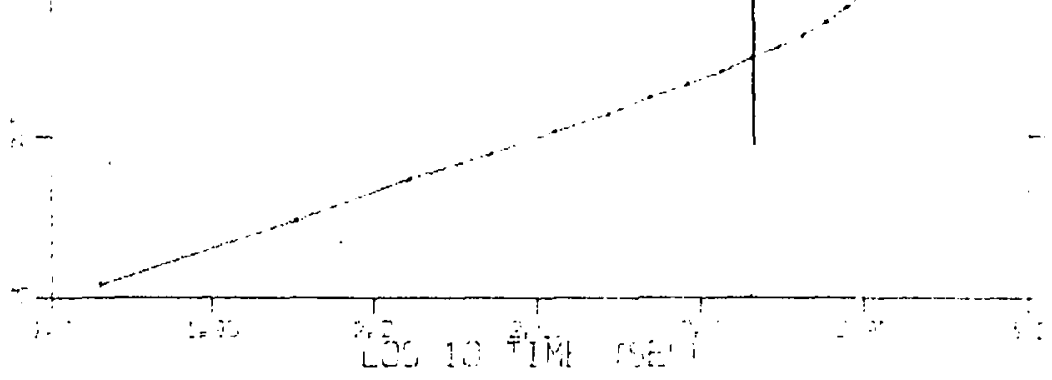

Figure B-27. $\log _{10}$ Axial Strain as a Function of $\log _{10}$ Time (Sec.), Test 9, Stage 2 . 


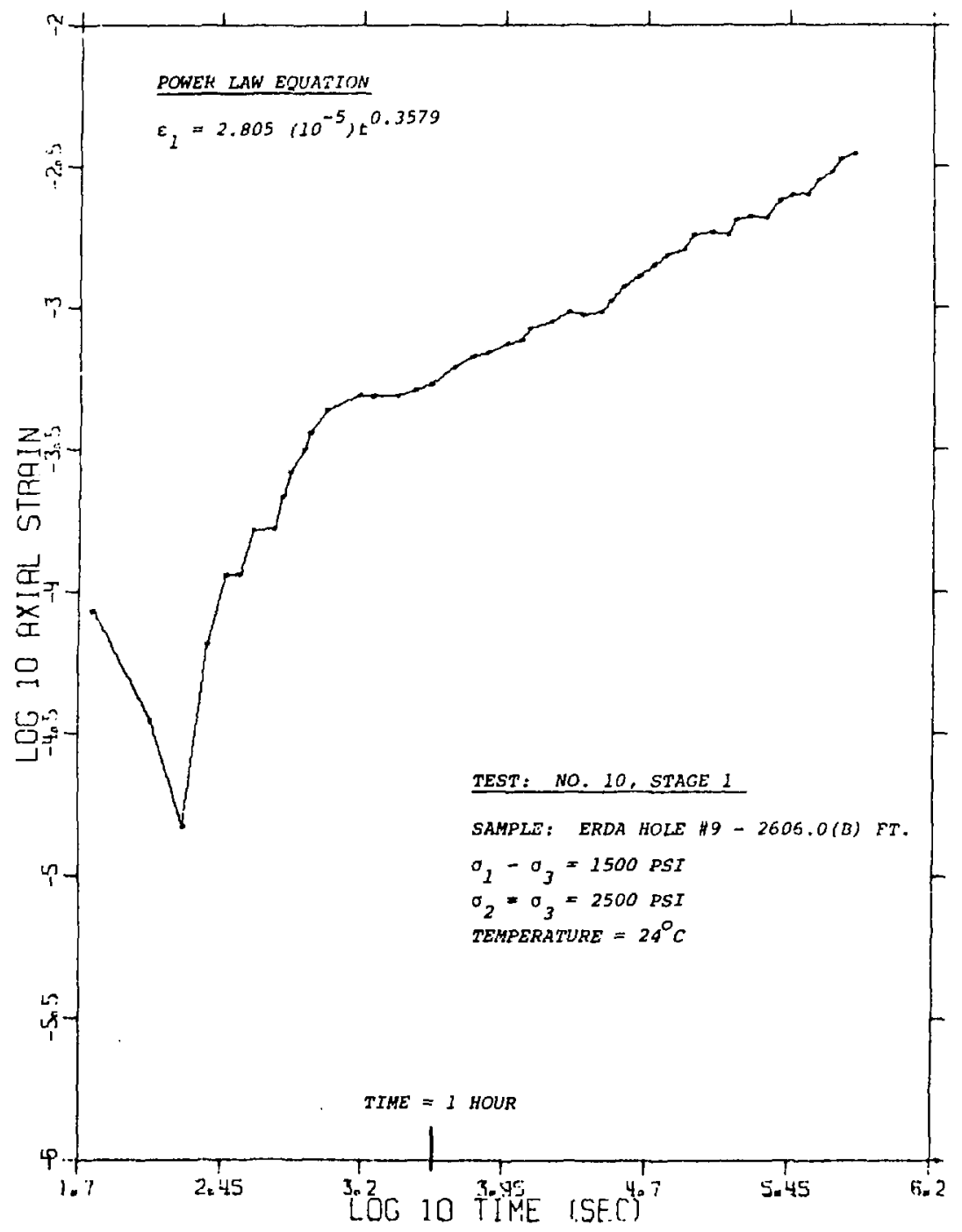

Figure B-28. $\log _{10}$ Axial Strain as a Function of $\log _{10}$ Time (Sec.). Test 10, Stage 1 . 


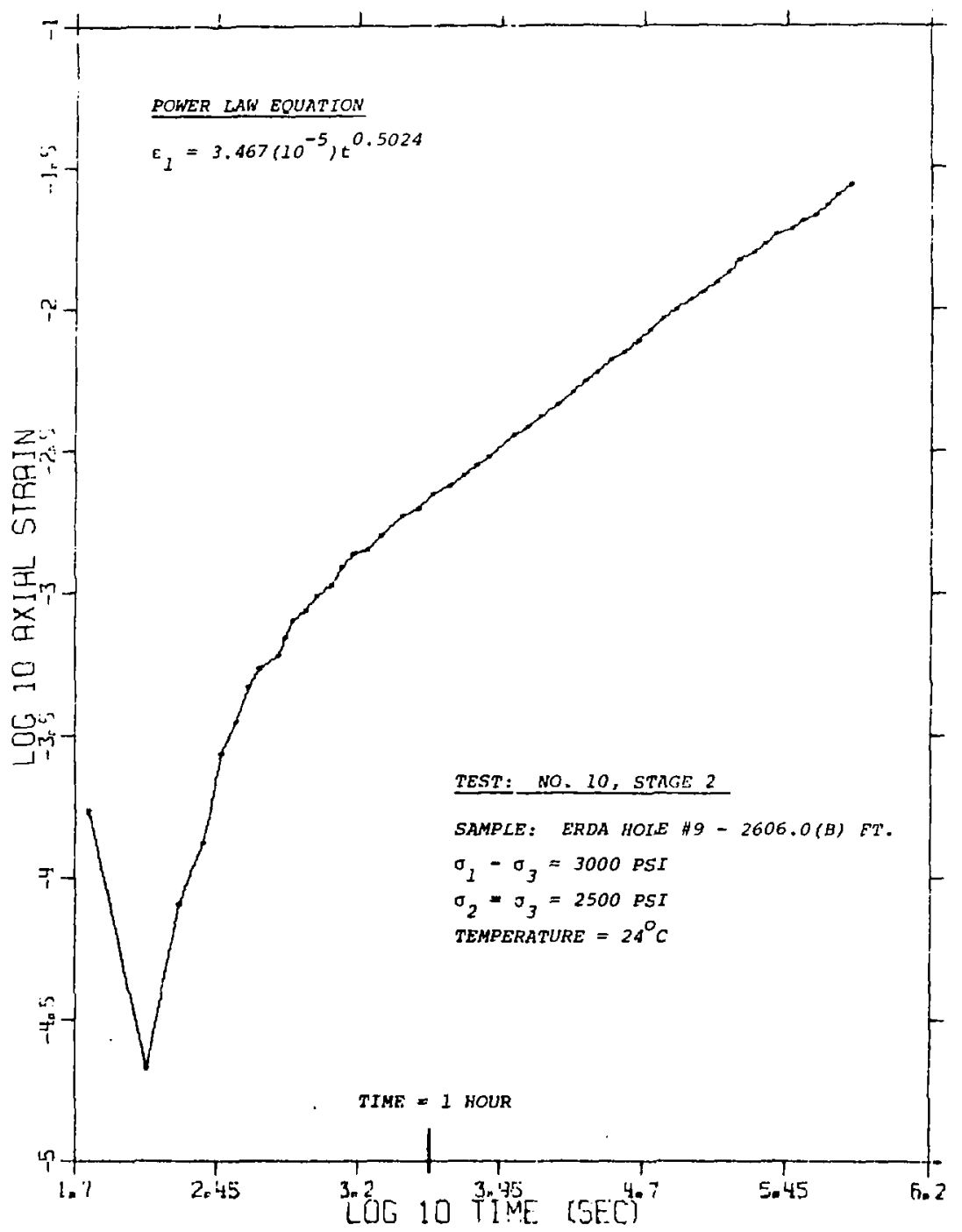

Figure $8-29 . \log _{10}$ Axial strain as a Function of $\log _{20}$ tinn (ste.), Test 10, Stage 2 . 


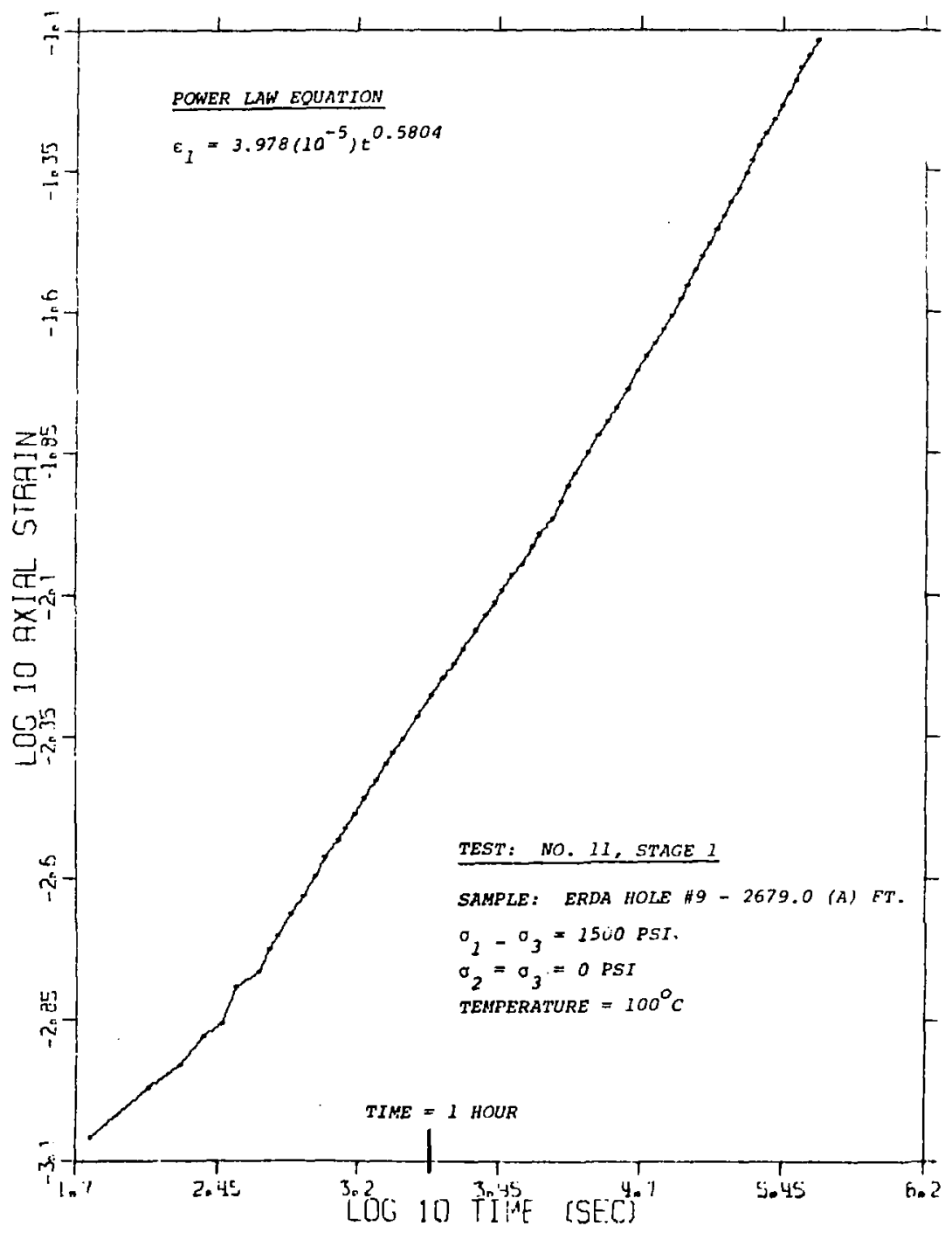

Figure B-30. $\log _{10}$ Axial strain as a Eunction of $\log _{10}$ Time (Sec.). Test 11, Stage 1 . 


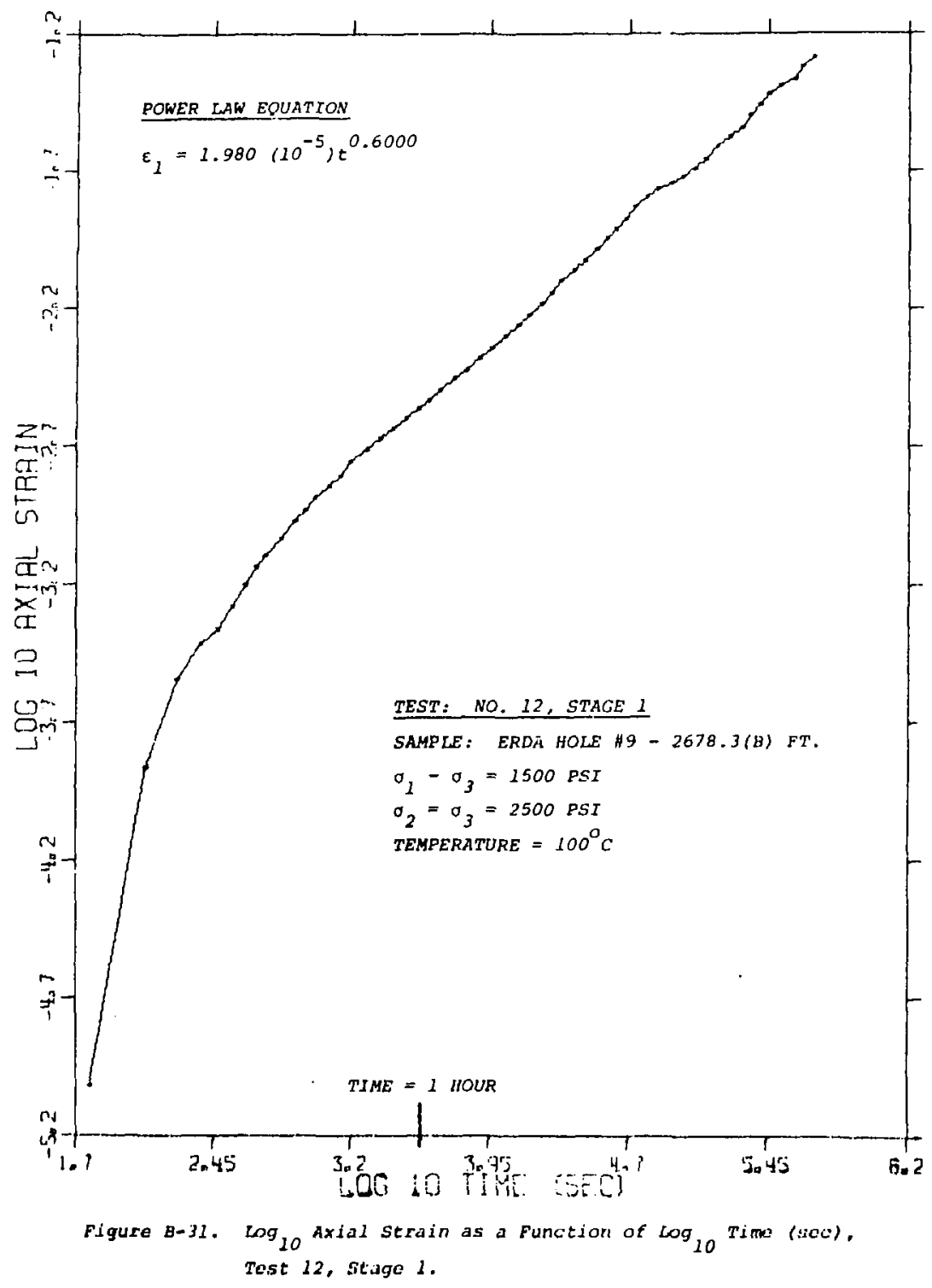




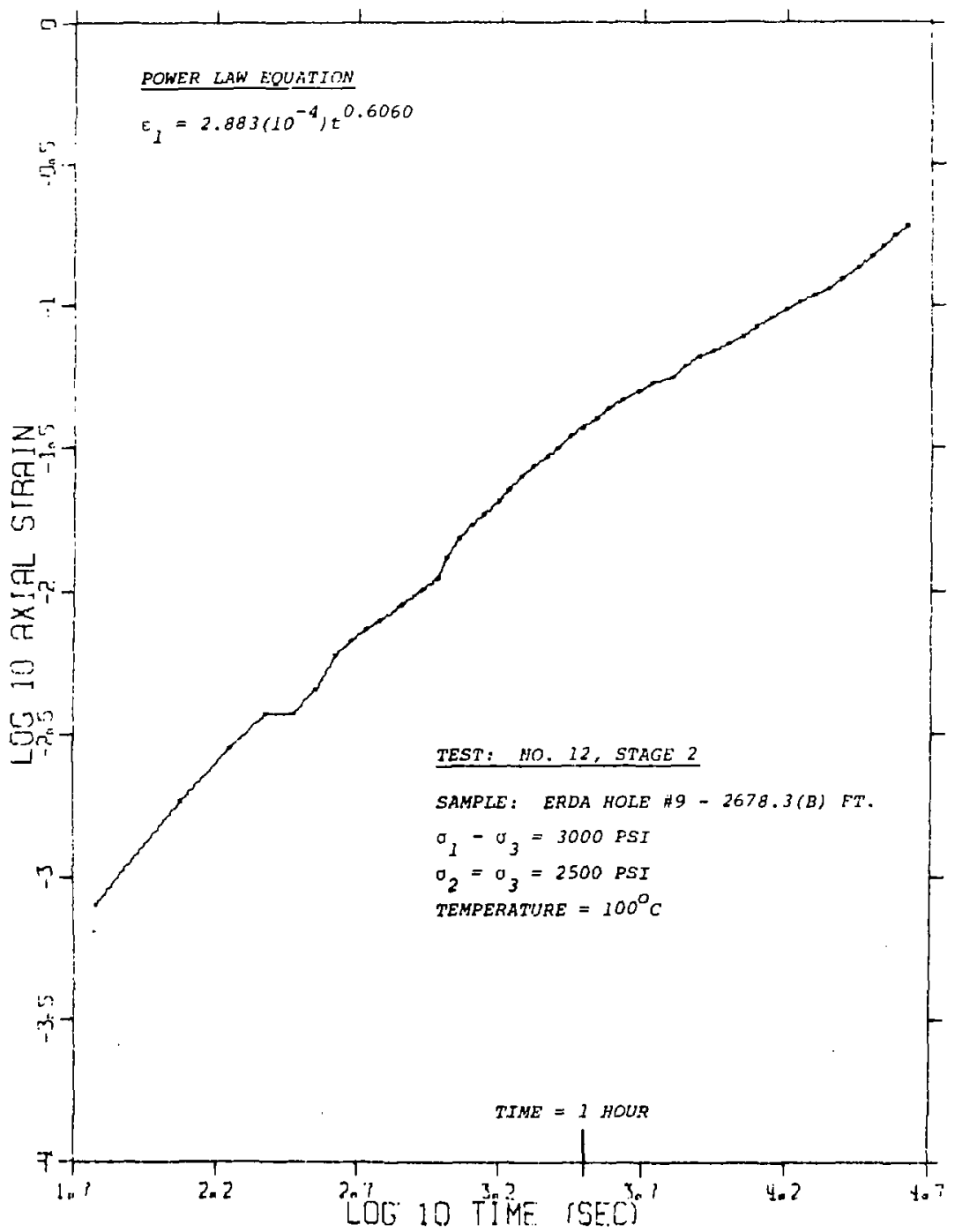

Figure 8-32: $\log _{10}$ Axial strain as a function of $\log _{10}$ Time (Sec.). Test 12 , stage 2.

96 


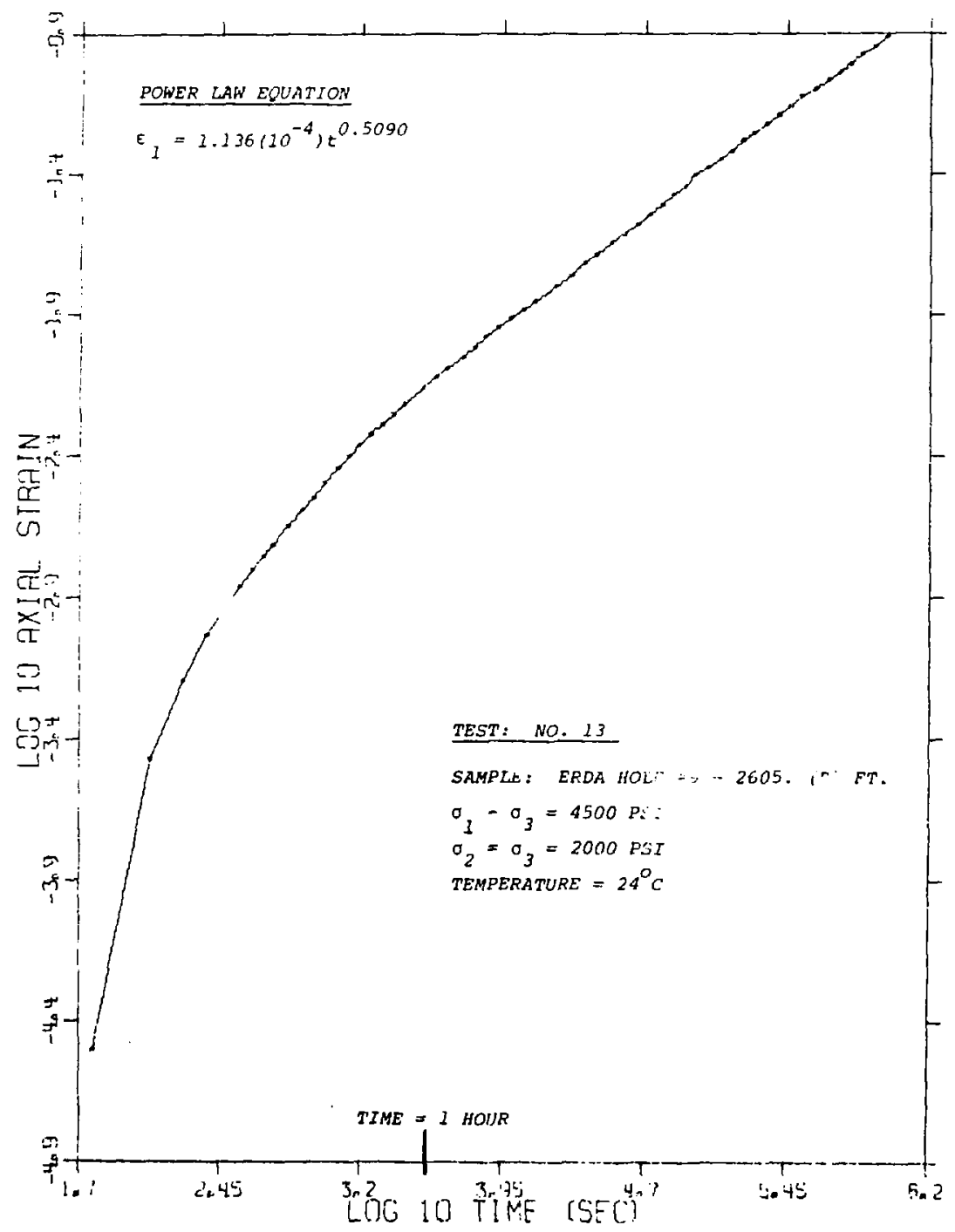

Figure $8-33 . \log _{10}$ Axial struin as a Function of $\log _{10}$ Time (Sec.). Test 13. 


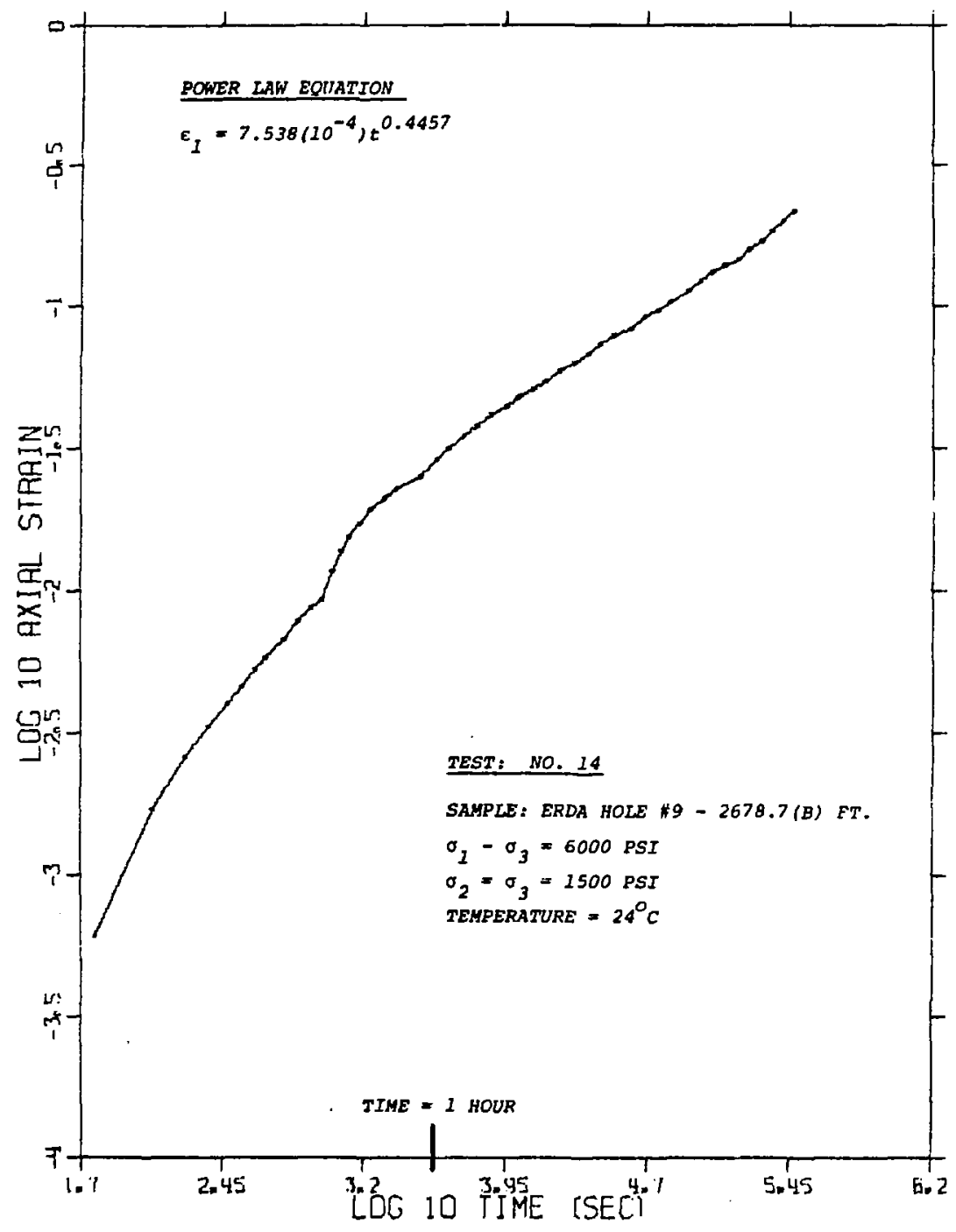

Figure B-34. $\log _{10}$ Axial strain as a Function of $\log _{10}$ Tine (Sec.). jest 14. 


\section{APPENDIX C}

PLOTS OF LATERAL STRATN

AS A FUNCTION OF TIME

FTGURE NO.

PLOTTED PARAHETERS

PAGE NO.

$c-1$

Lateral strain as a Function of Time, Test 5

C-2 Lateral strain as a Function of Time, Test 6

Lateral Strain as a Function of mime, Test 7

Lateral strain as a Function of Time, Test 8

c-5 Lateral strain as a Function of Time, Test 10,

C-8 Lateral Strain as a Function of Time, Test 12. Stage 2

c-10 Lateral strain as a Function of Time, Test 14 


\section{APPENDIX C (CONT'D)}

FTGURE NO.

$c-19$

$c-20$

\section{PLOTTED PARAMETERS}

PAGE NO.

$\log _{20}$ Lateral Strain as a Function of $\log _{10}$ Time (Set.), Test 13

$\log _{10}$ Lateral strain as a Function of $\log _{10}$ Time (Sec.), Test 14
119

120 


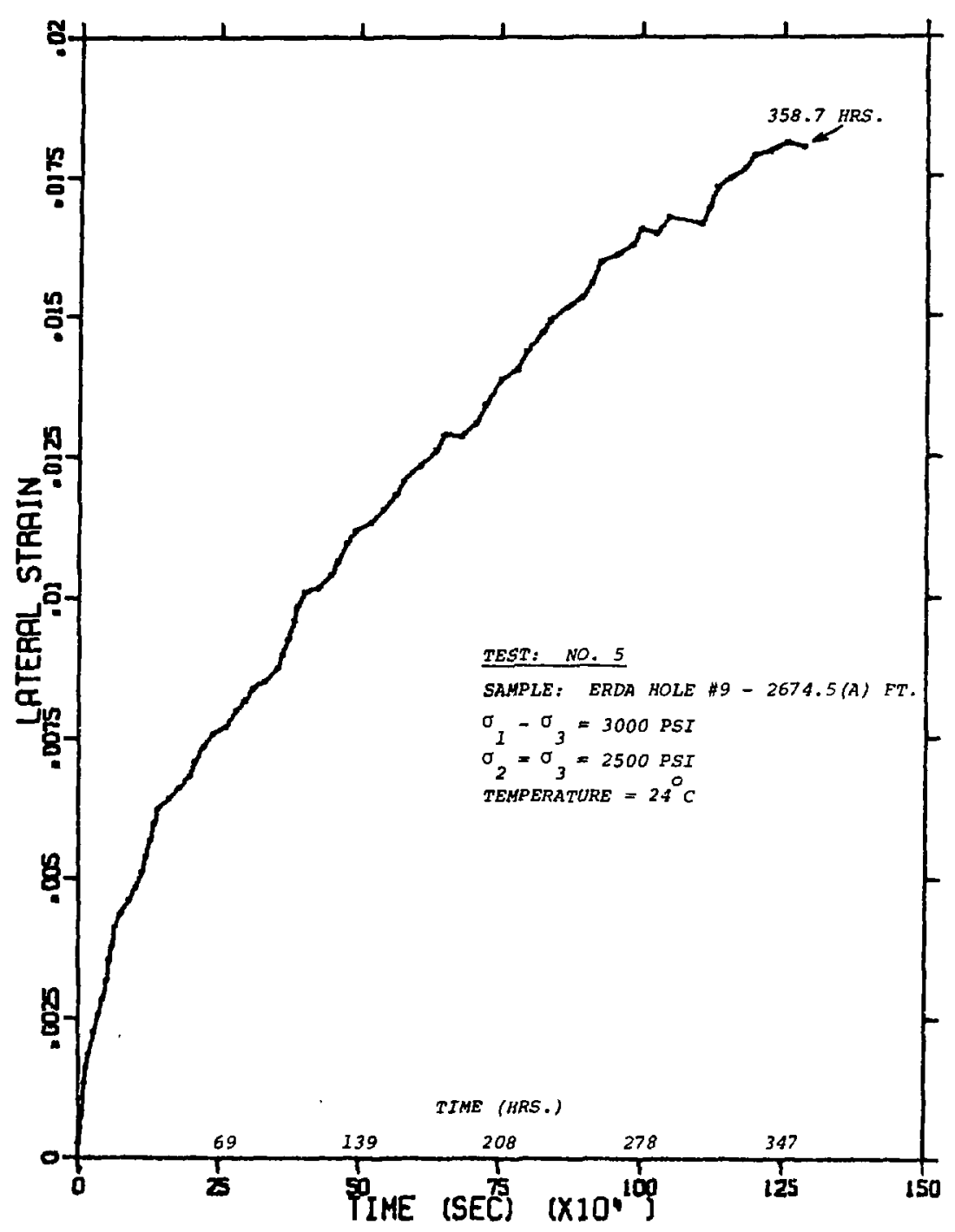

Figure c-1. Lateral strain as a Function of Time. Test 5. 


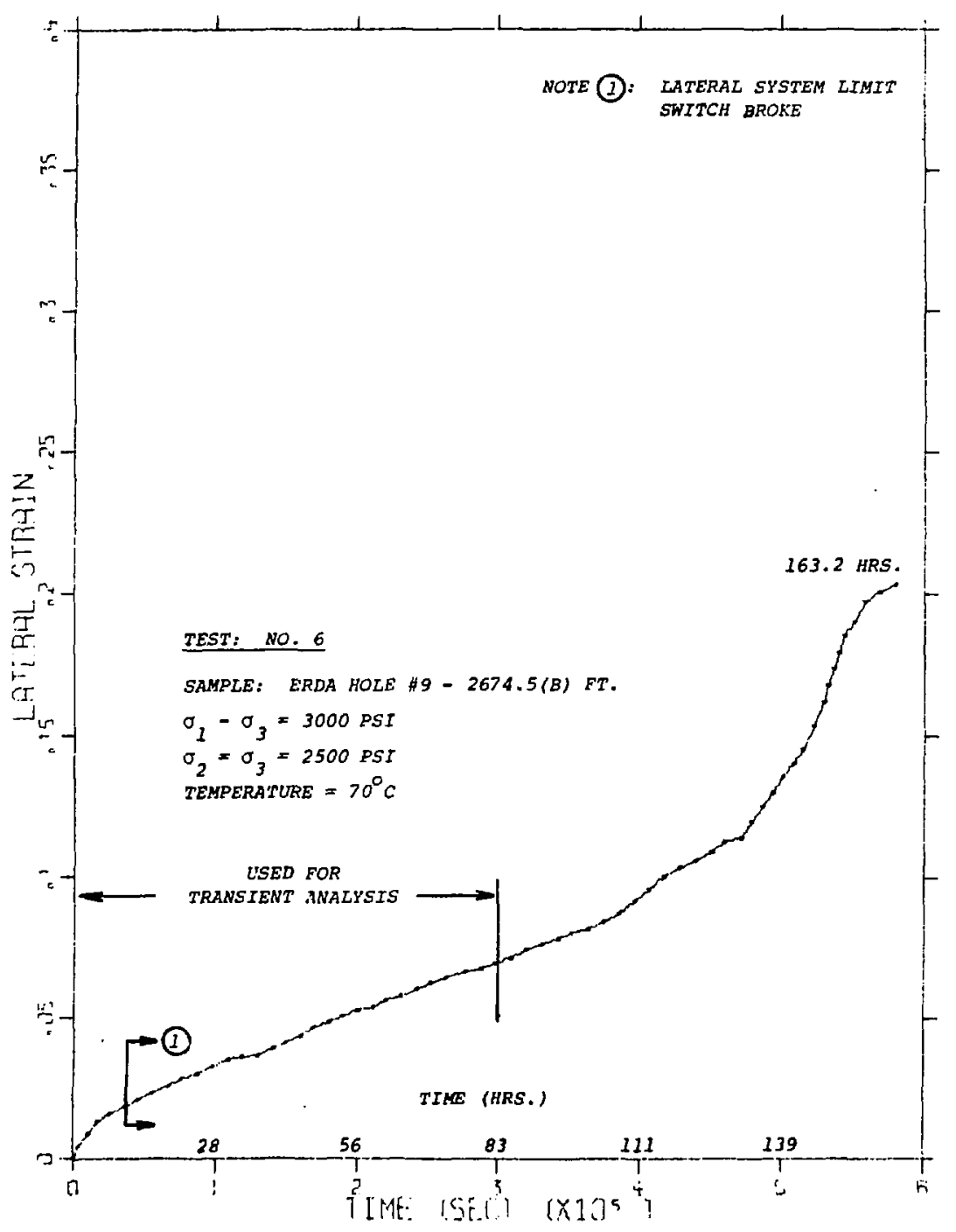

Fighre C-2. Lateral Strain as a Function of Time, Test 6. 


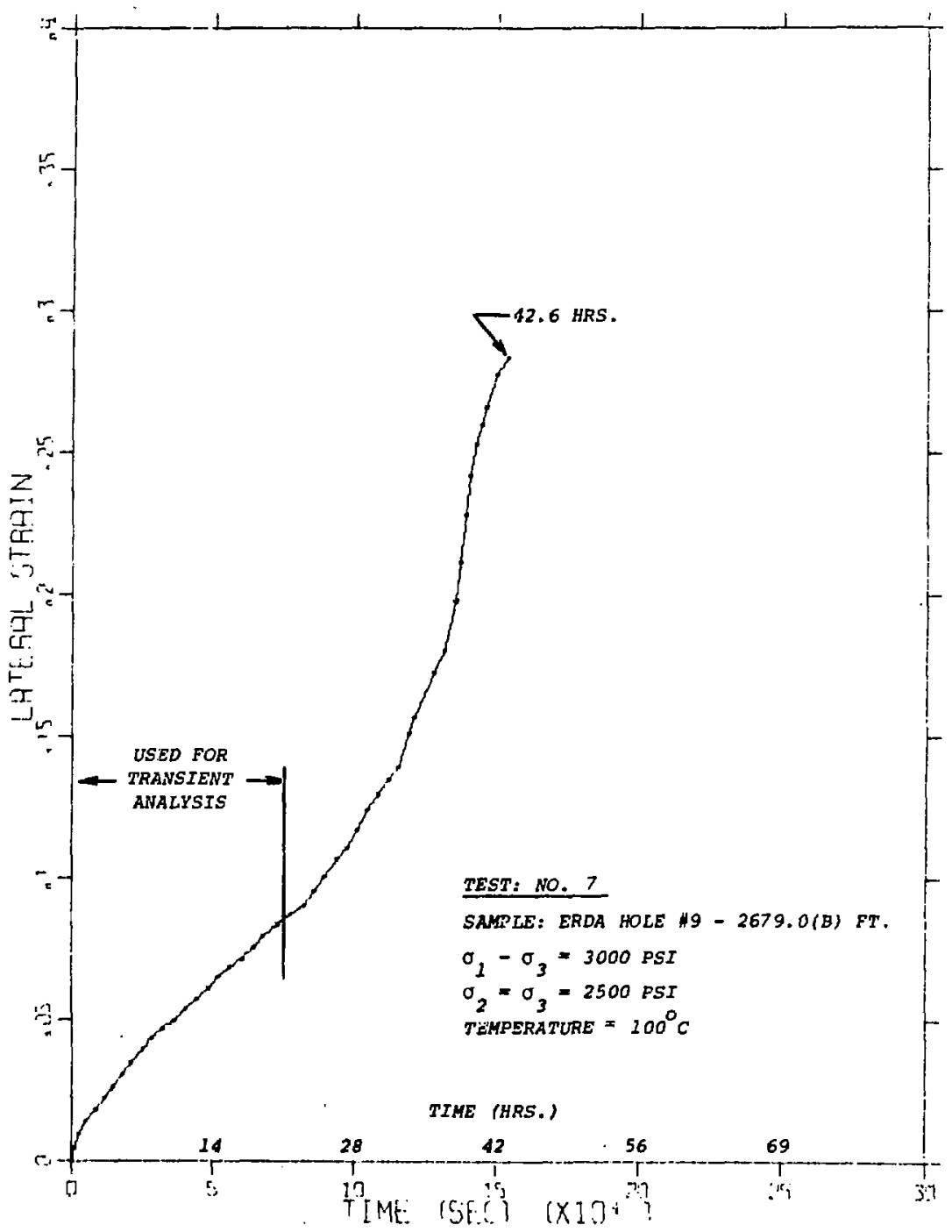

Figure c-3. Lateral strain as a Function of rime, Test 7. 


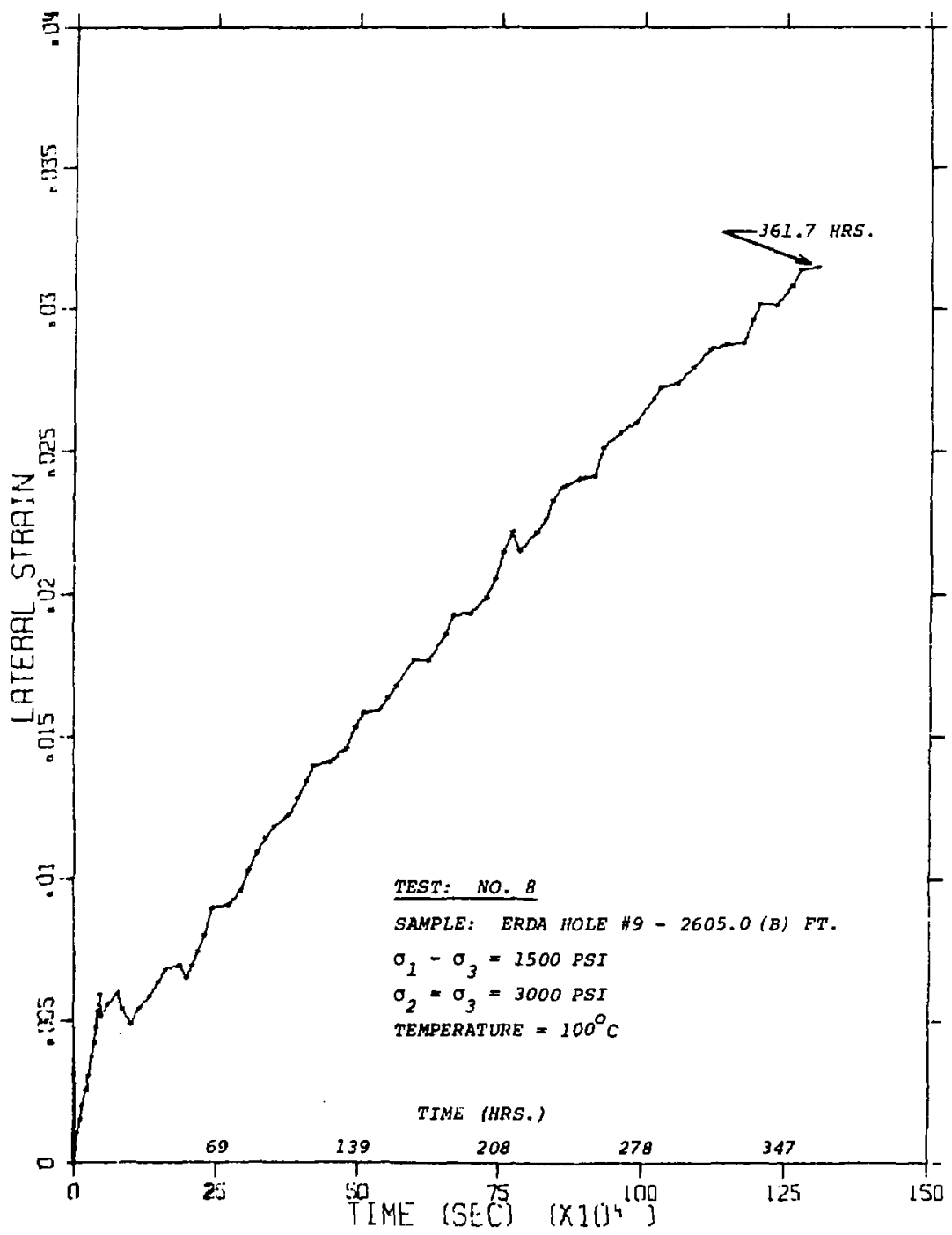

Figure C-4. Lateral Strain as a Function of Time, Test \&. 


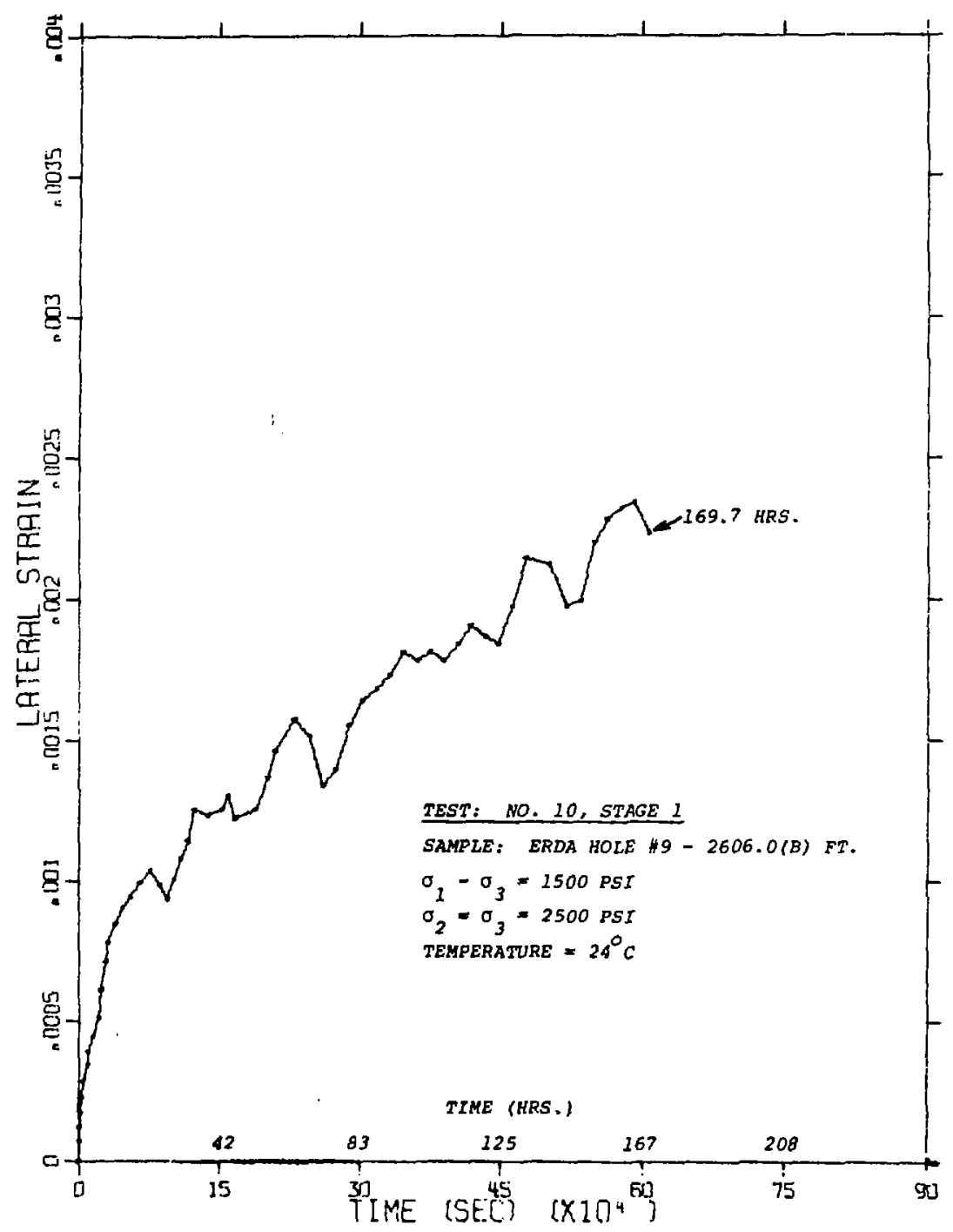

Figure C-5. Lateral Strain as a Function of Time, Test 1C, Stage 1. 


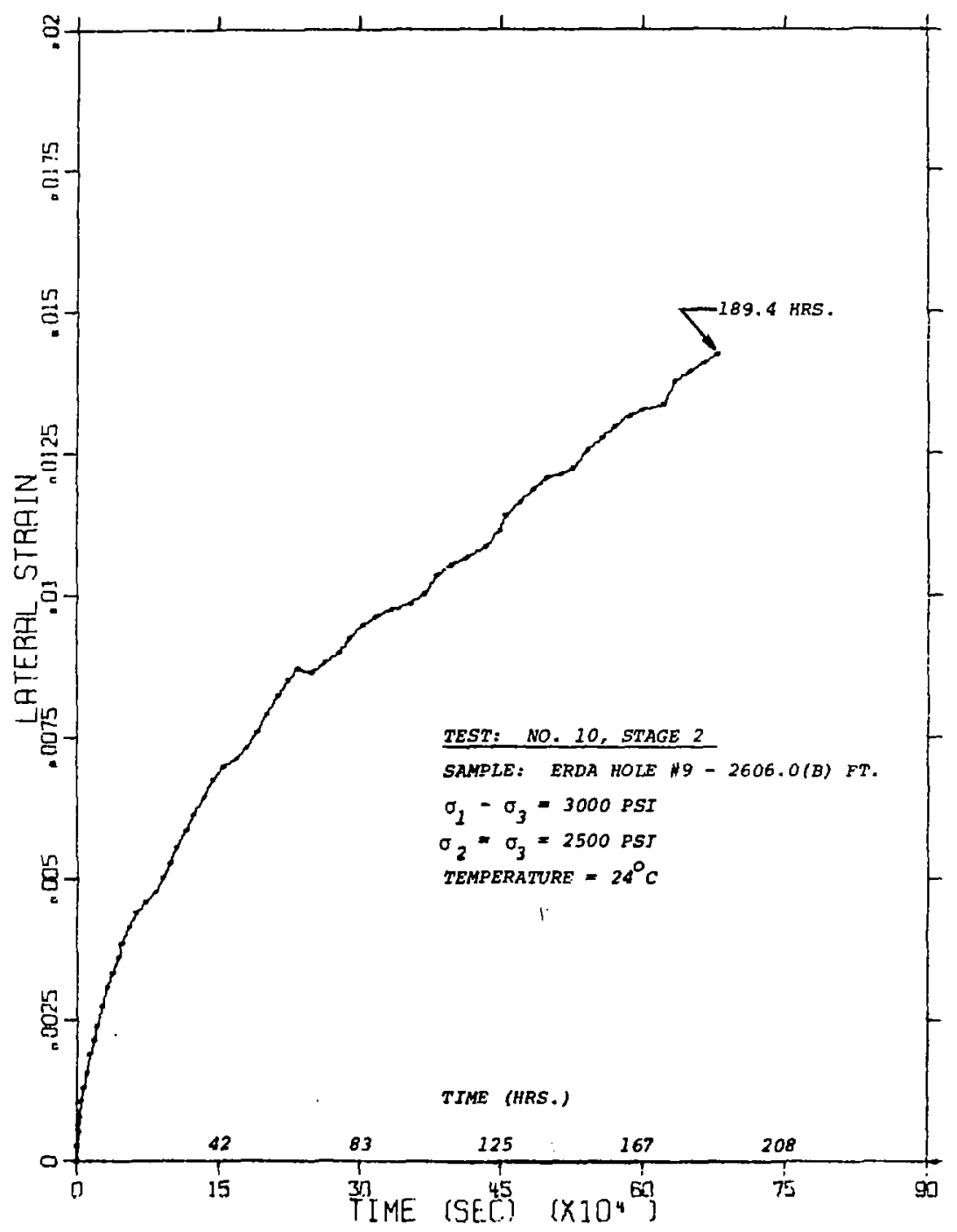

Figure c-6. Lateral Strain as a function of Time, Test 10, Stage 2. 


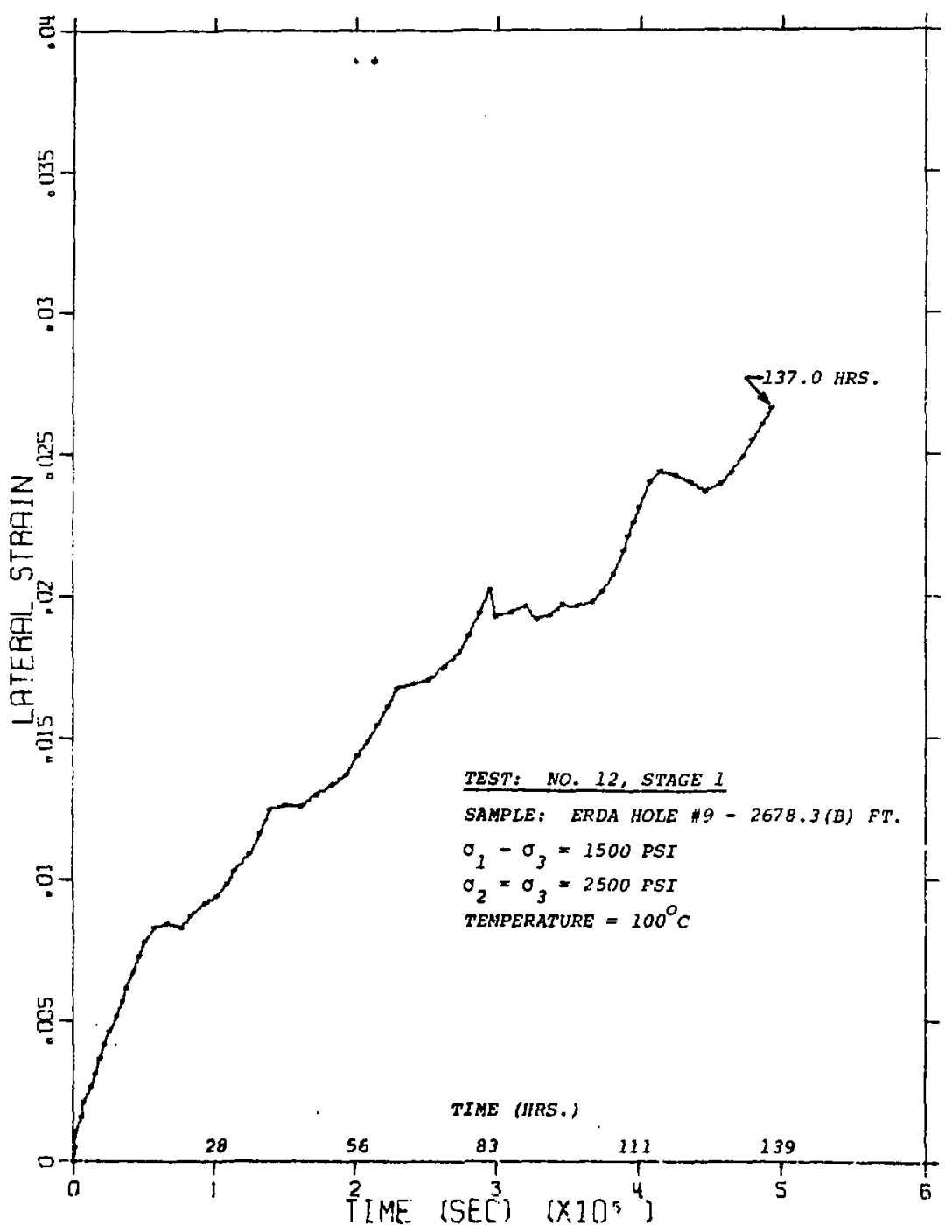

Figure c-7. Lateral strain as a Function of Time, Test 12 , Stage 1 


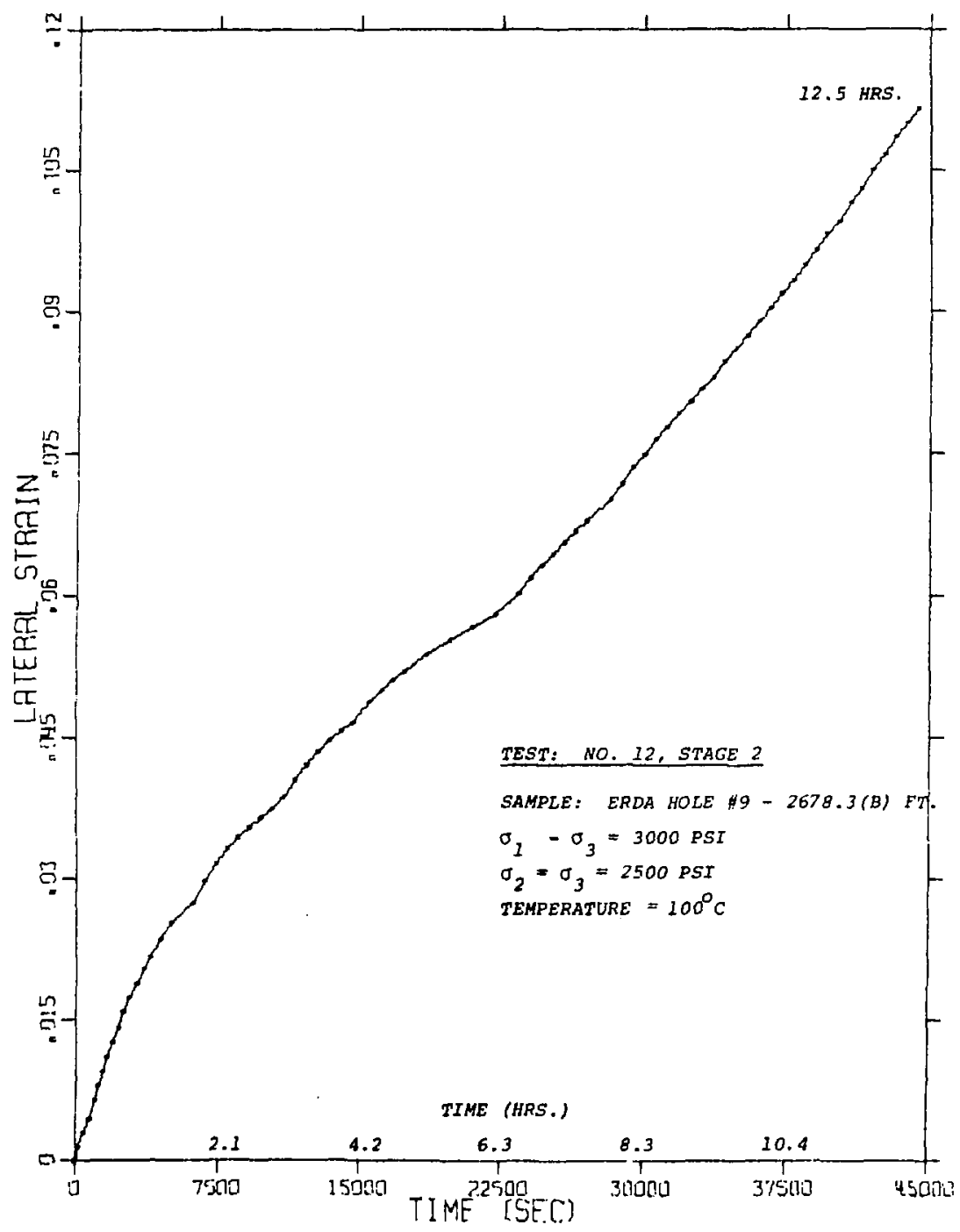

Figure C-8. Lateral strain as a Function of time, Test 12, stage 2. 


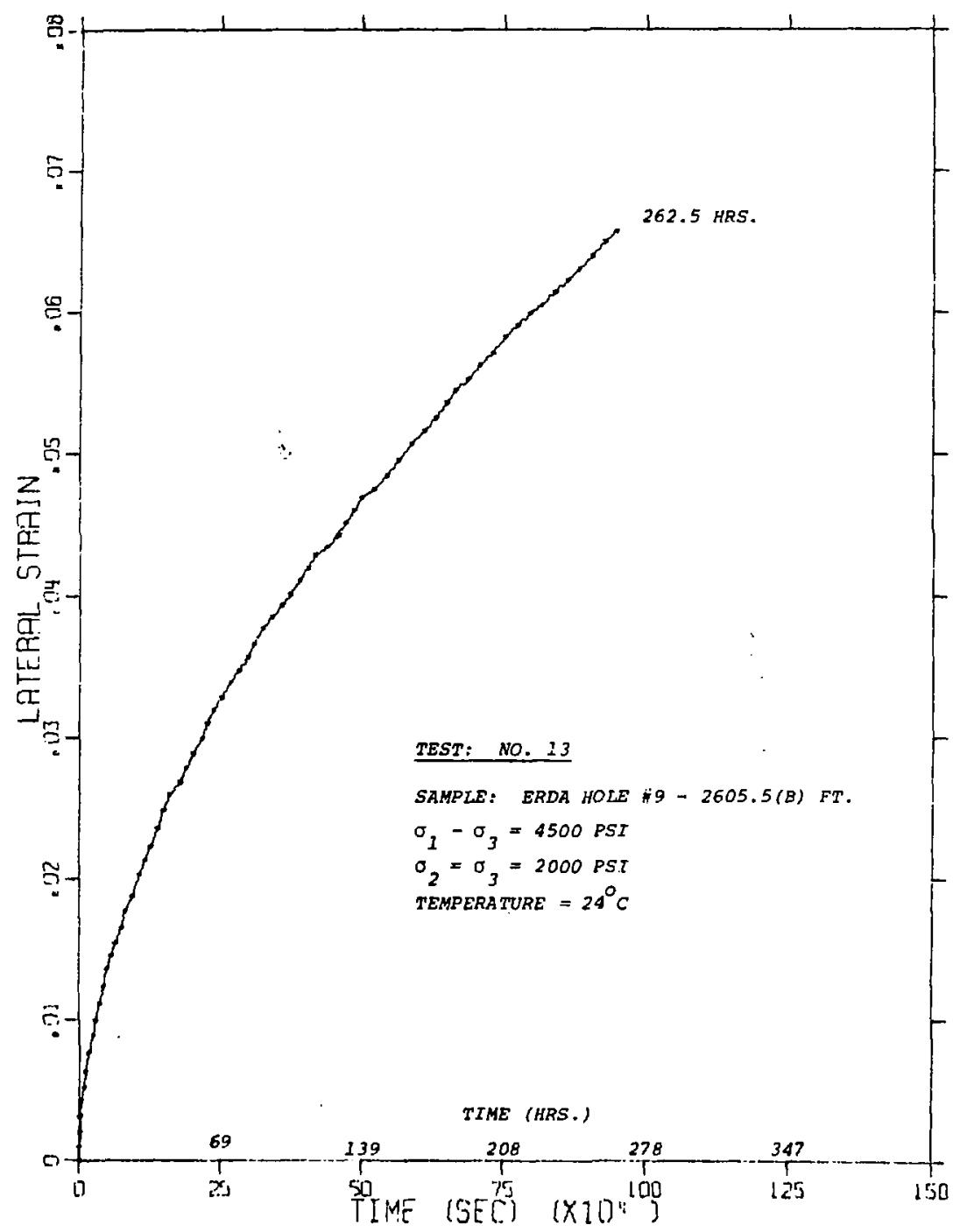

Figure C-9. Lotural strain as a Function of time, Test 13. 


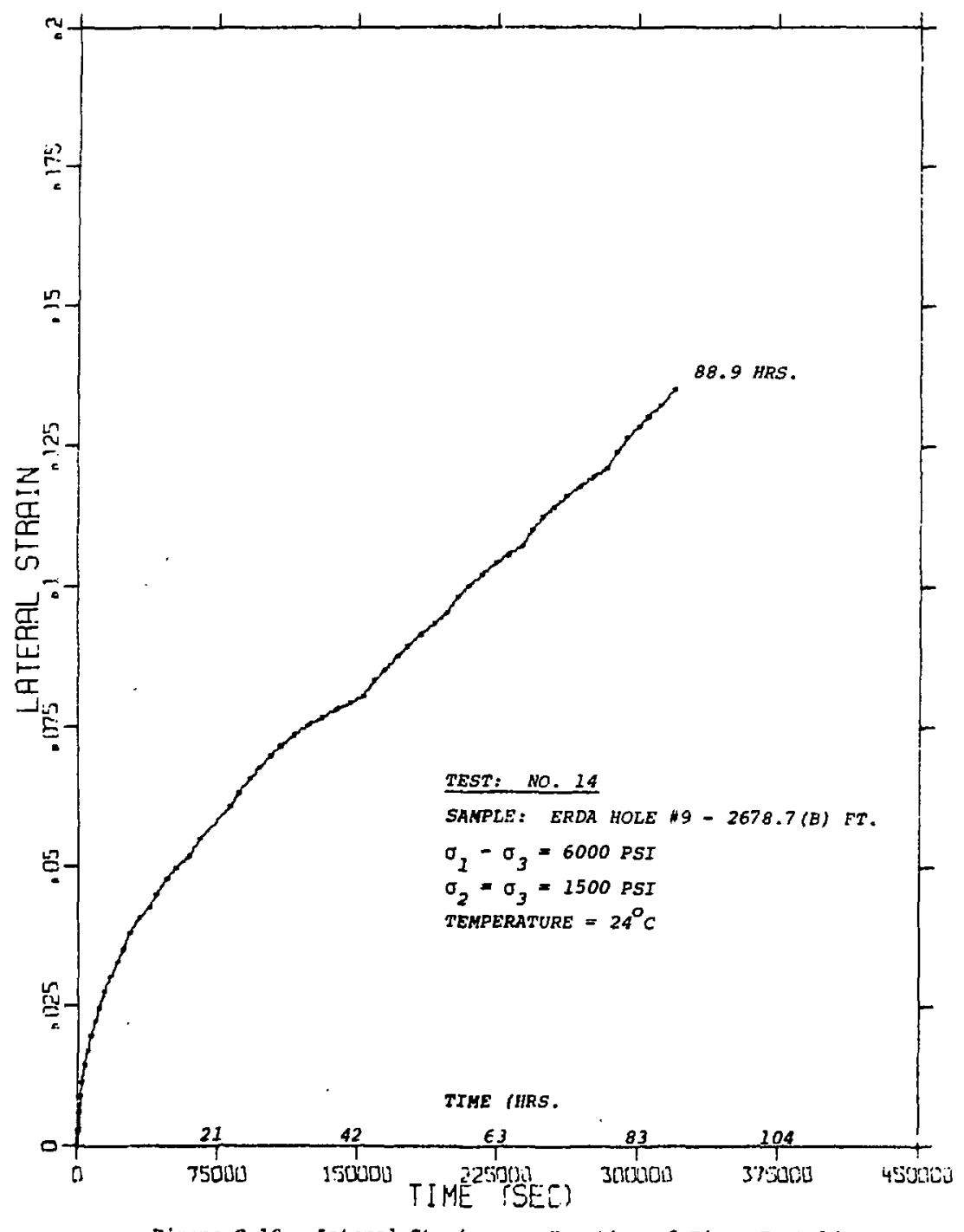

Figure c-10. Lateral strain as a Function of Time, Test 14. 


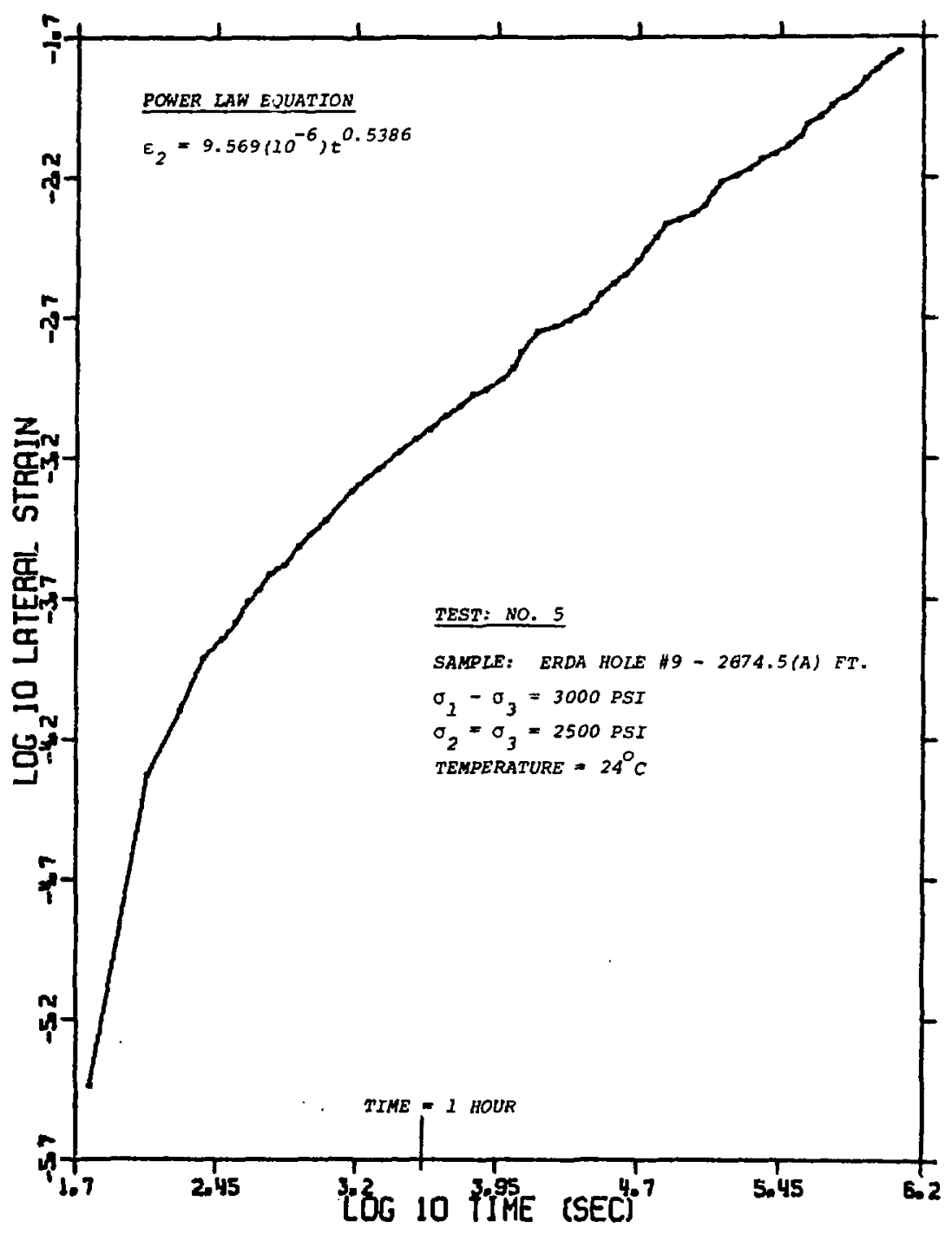

Figure C-11. $\log _{10}$ Lateral Strain as a Function of $\log _{10}$ Time (Sec.), Test 5. 


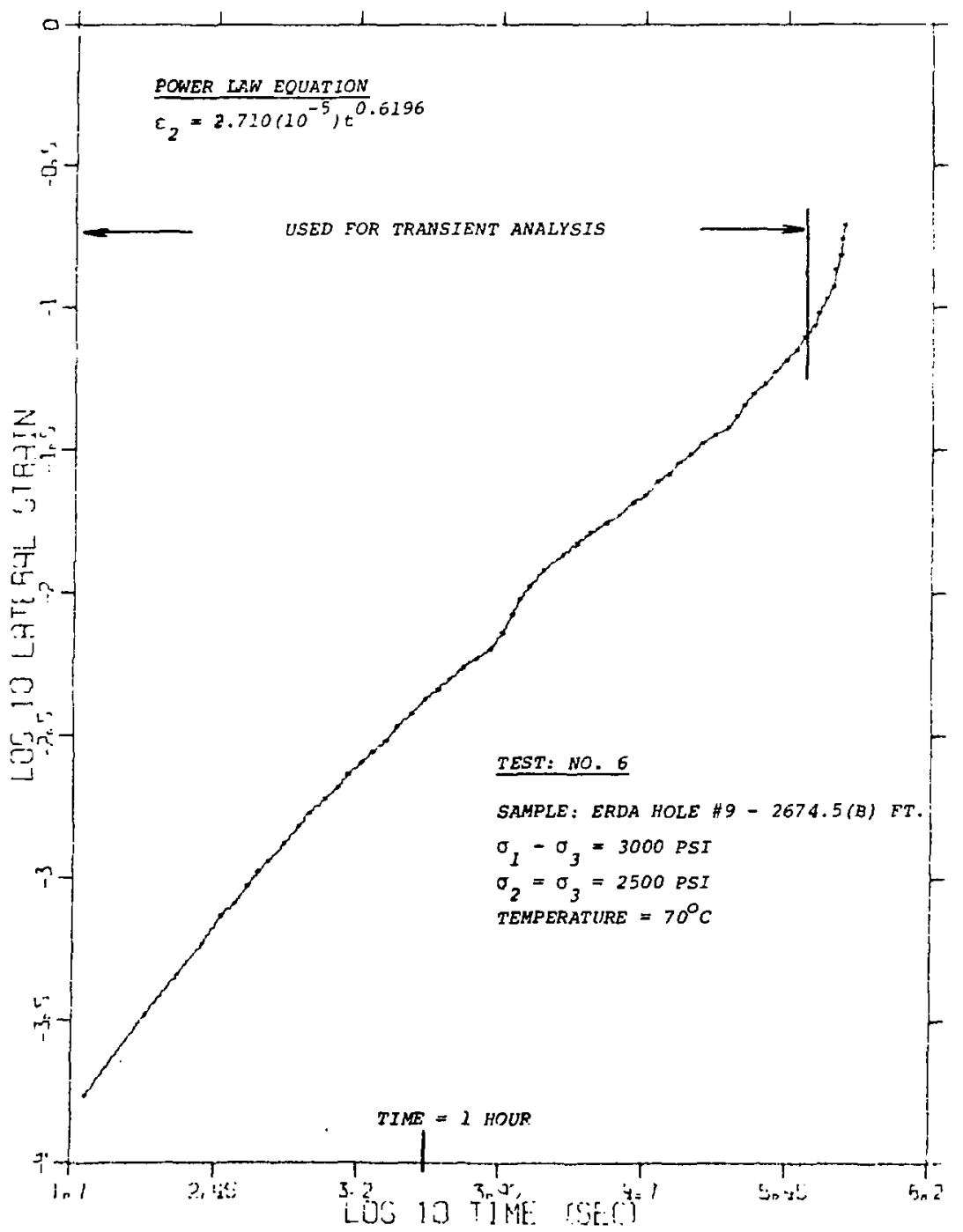

Figure $C-12 . \log _{10}$ Lateral Strain as a Function of $\log _{10}$ Time (Sec.), rest 6 .

112 


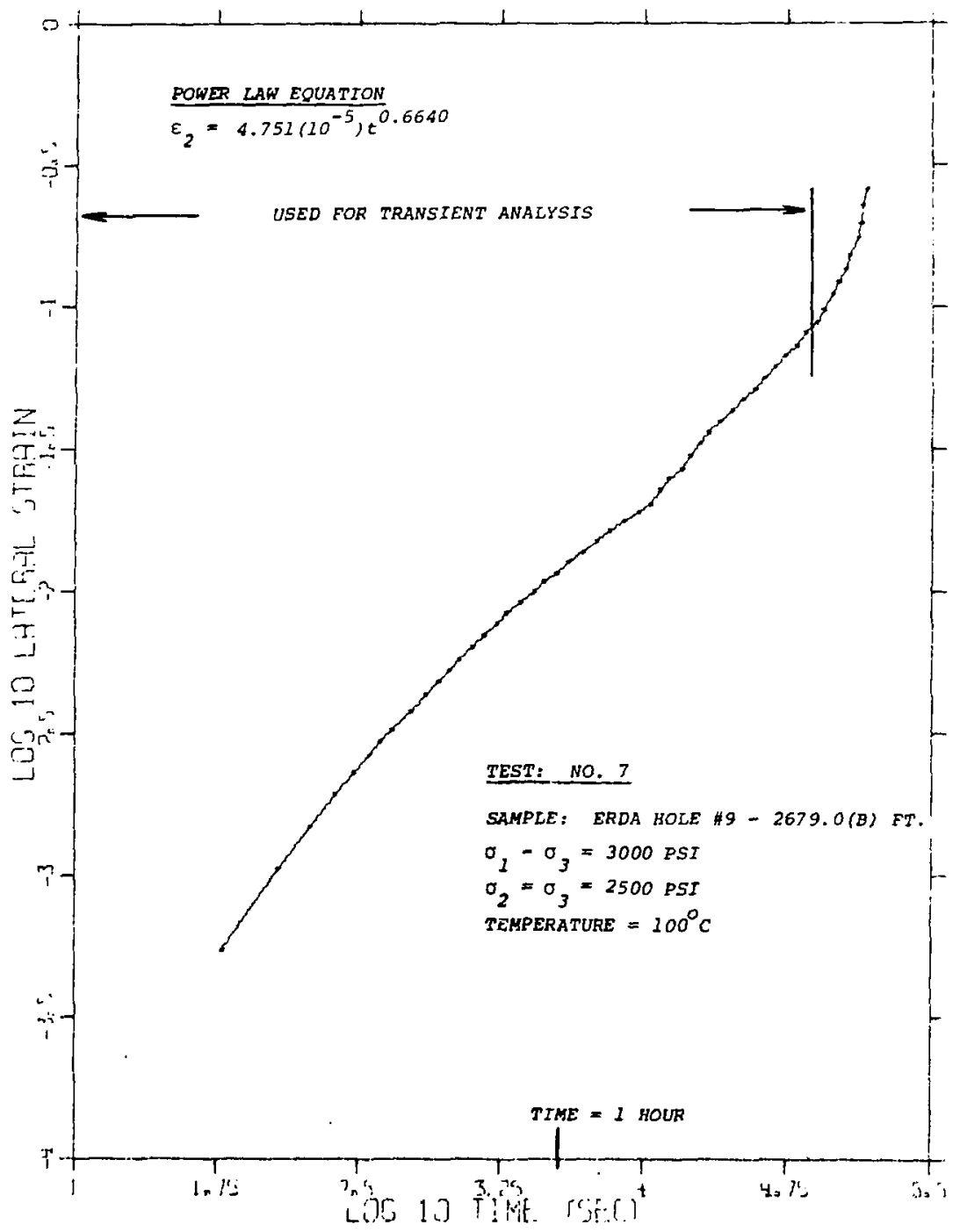

Figure $6-13 . \log _{10}$ Lateral Strain as a Function of $\log _{10}$ Time (Sec.). rest 7.

113 


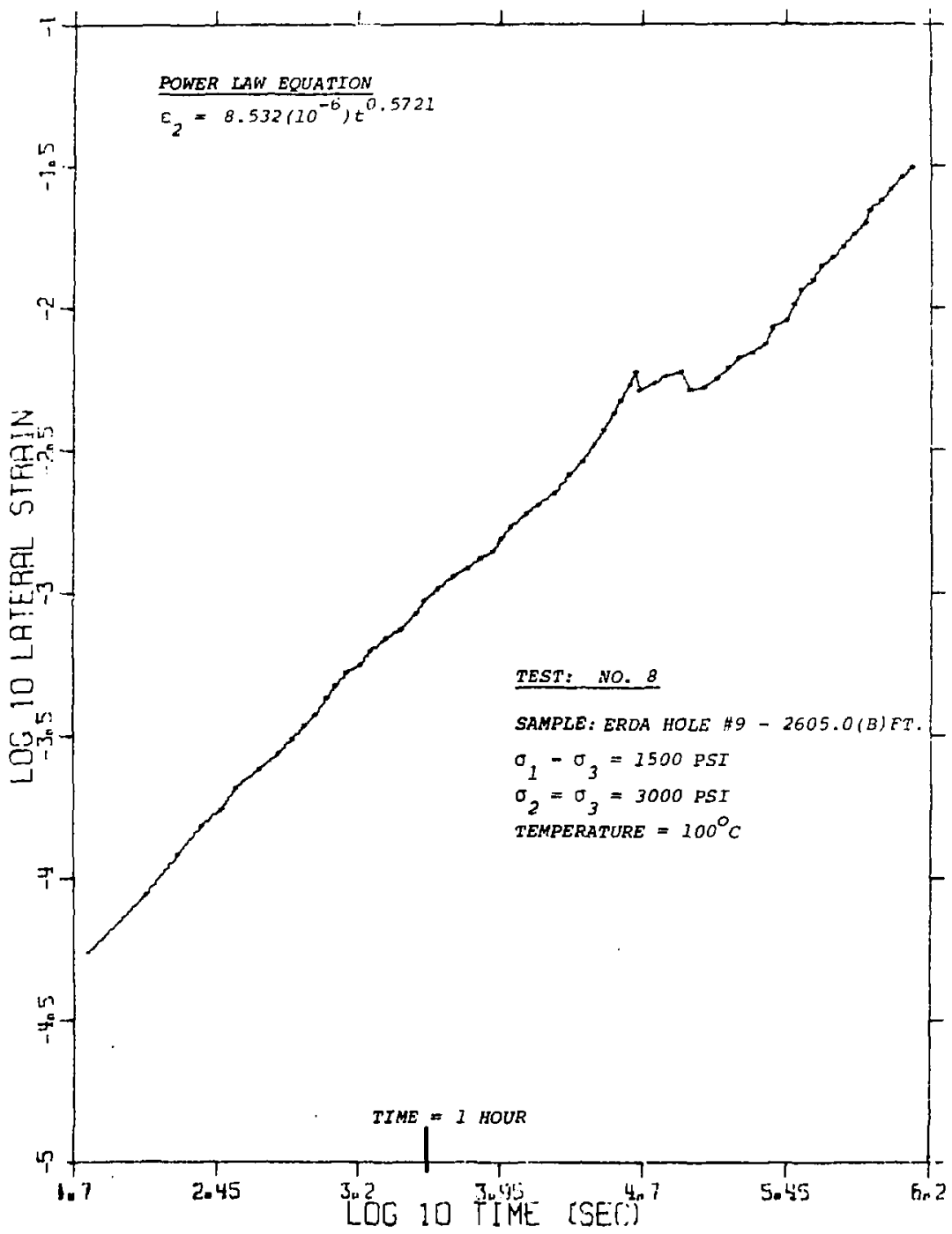

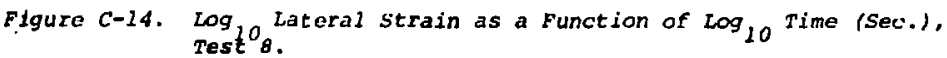




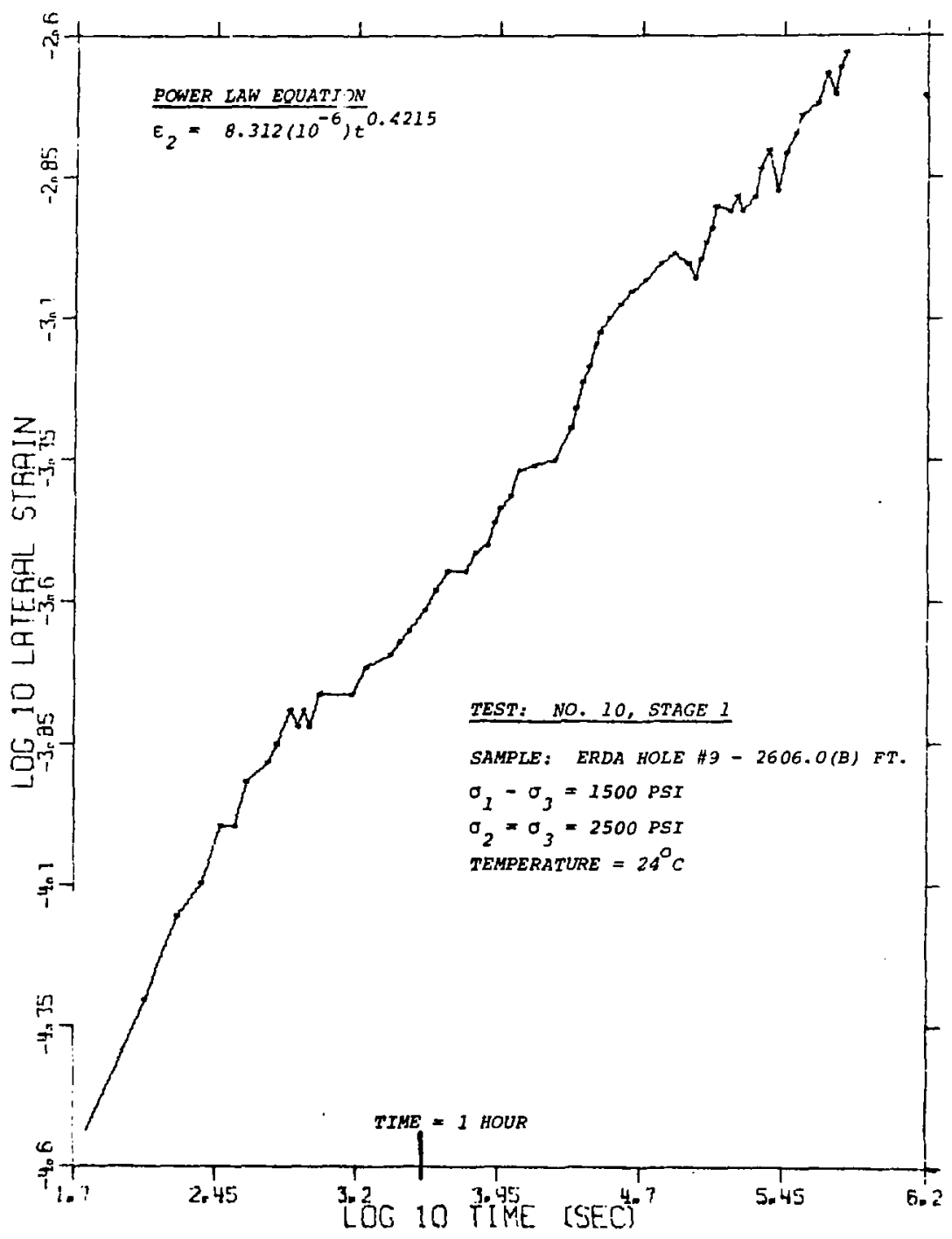

Figure C-15. $\log _{10}$ Lateral strain as a Function of $\log _{20}$ Time (Sec.). Test 10, stage 1 . 


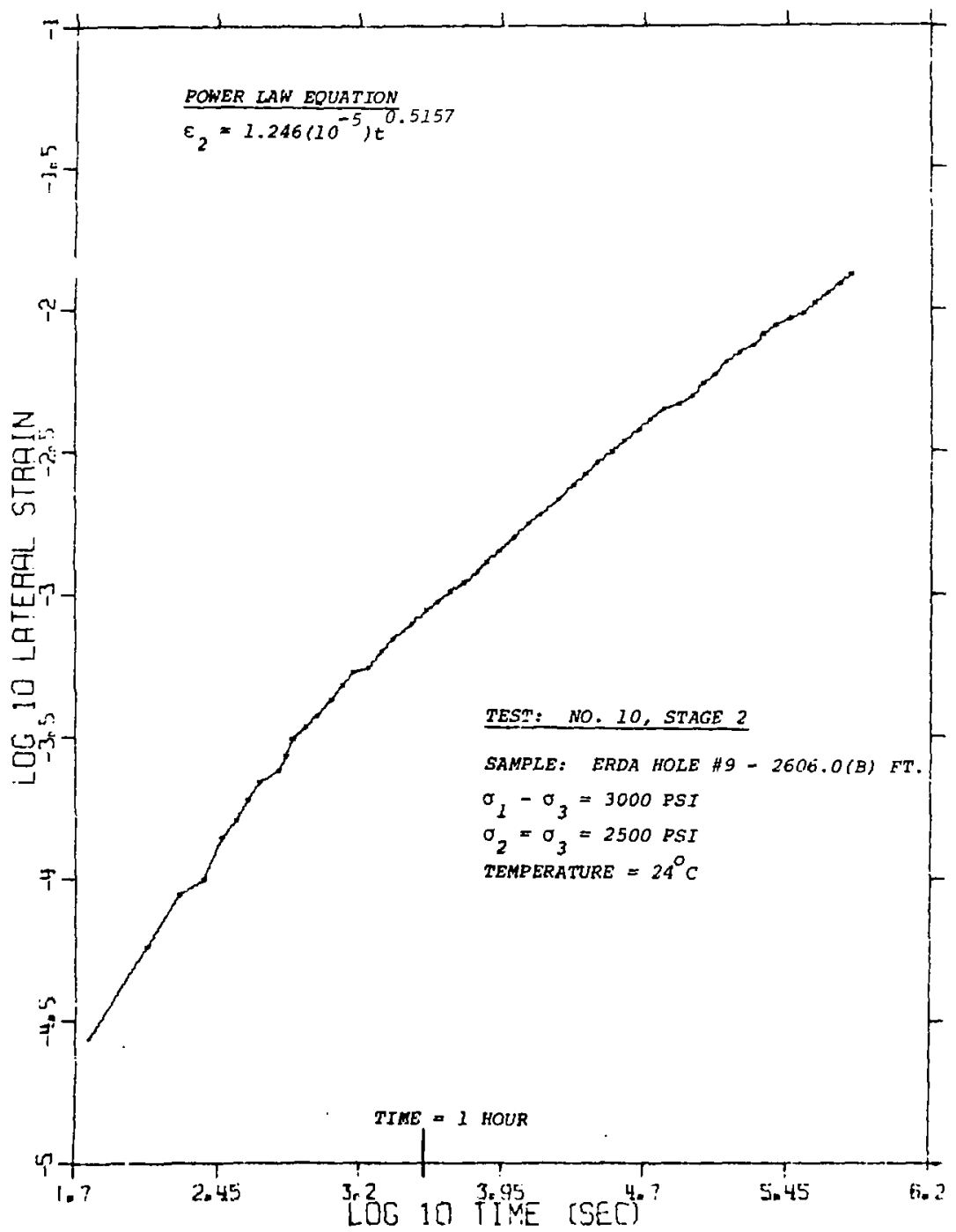

Figure C-16. $\log _{10}$ Lateral strain as a function of $\log _{20}$ Time (Sec.), Test 10 , stage 2. 


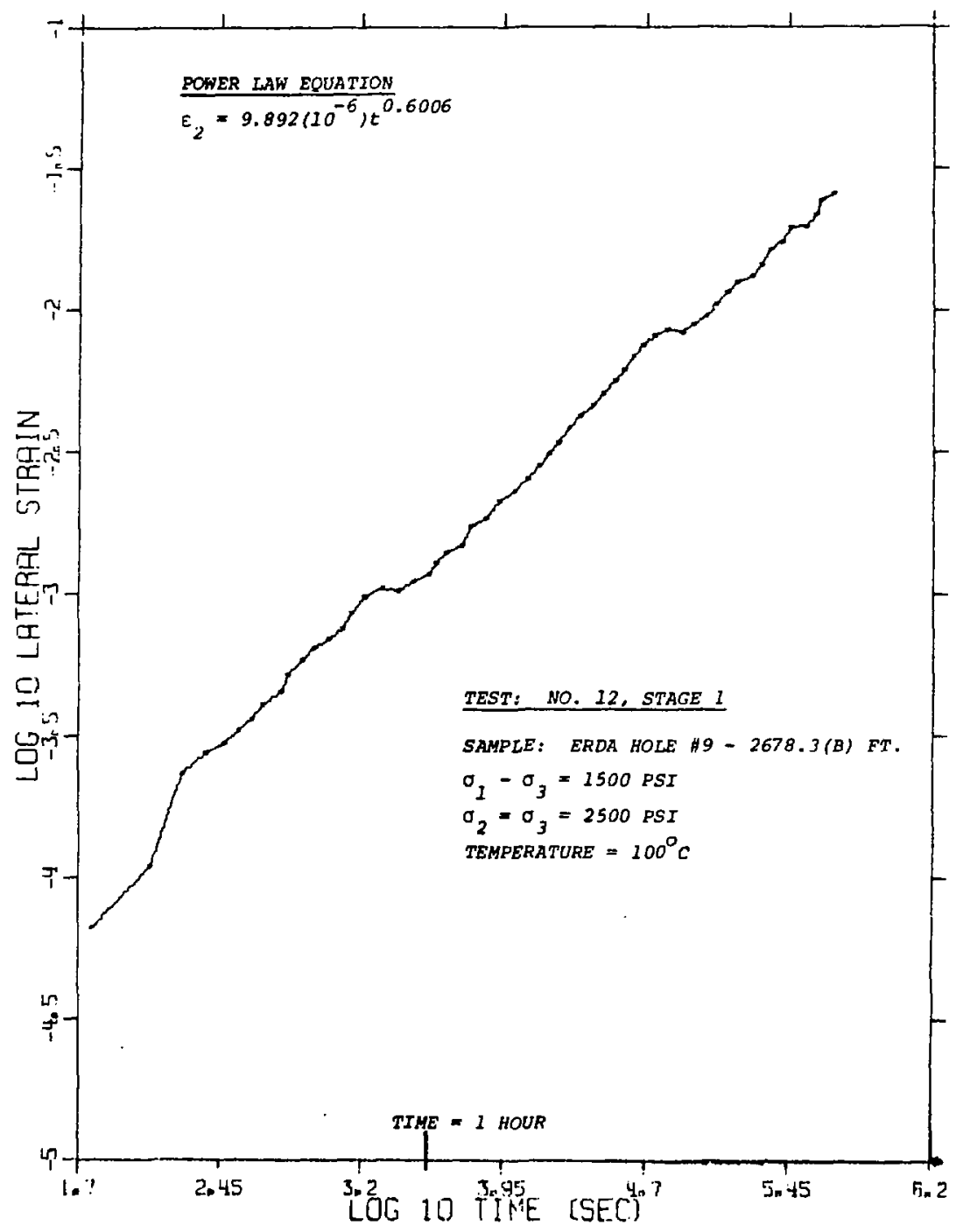

Figure $c-17 . \log _{10}$ tateral strain as a Function of $\log _{10}$ Time (Sec.), Test 12, stage 2. 


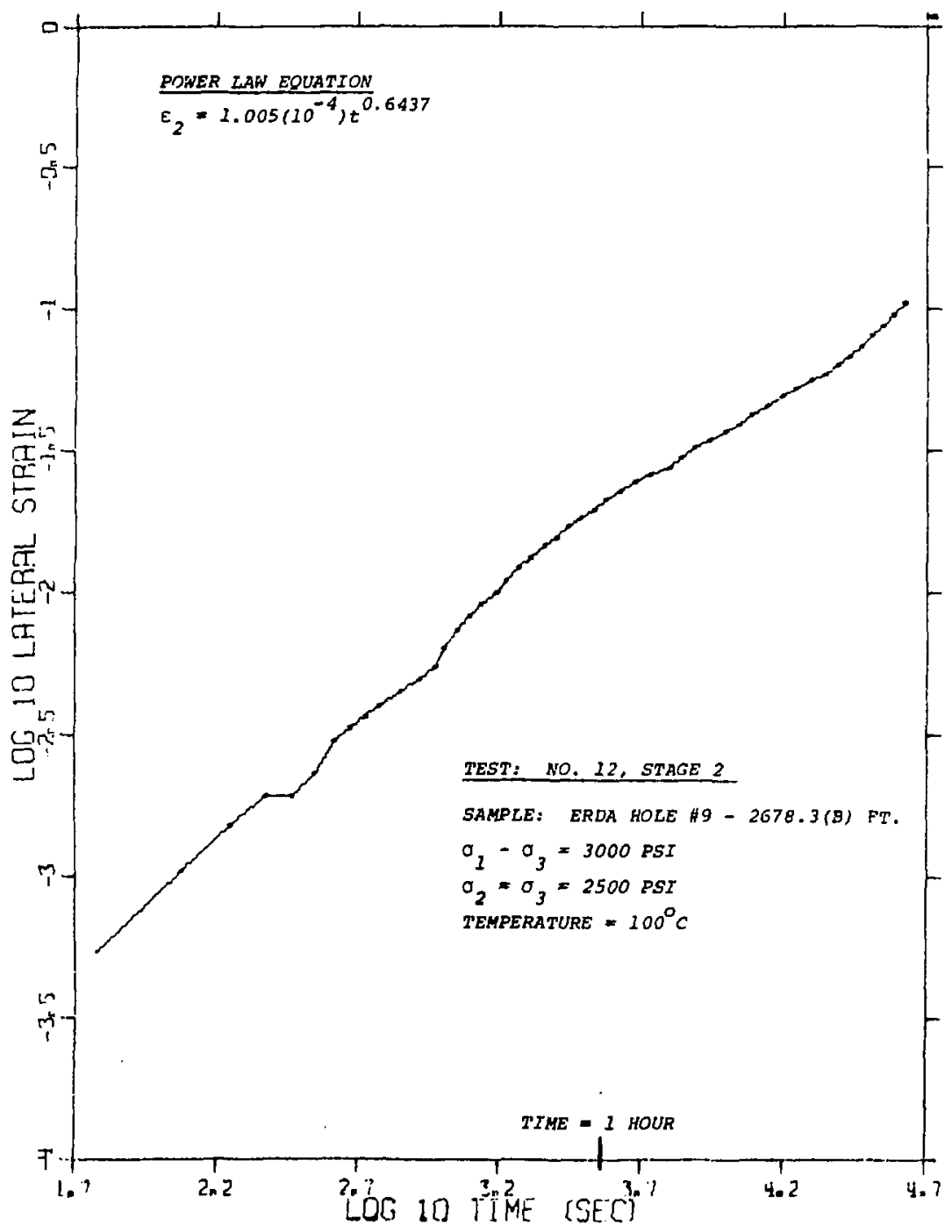

Figure $c-19 . \log _{10}$ Lateral strain as a Function of $\log _{10}$ Time (Sec.), rest 12, stage 2. 


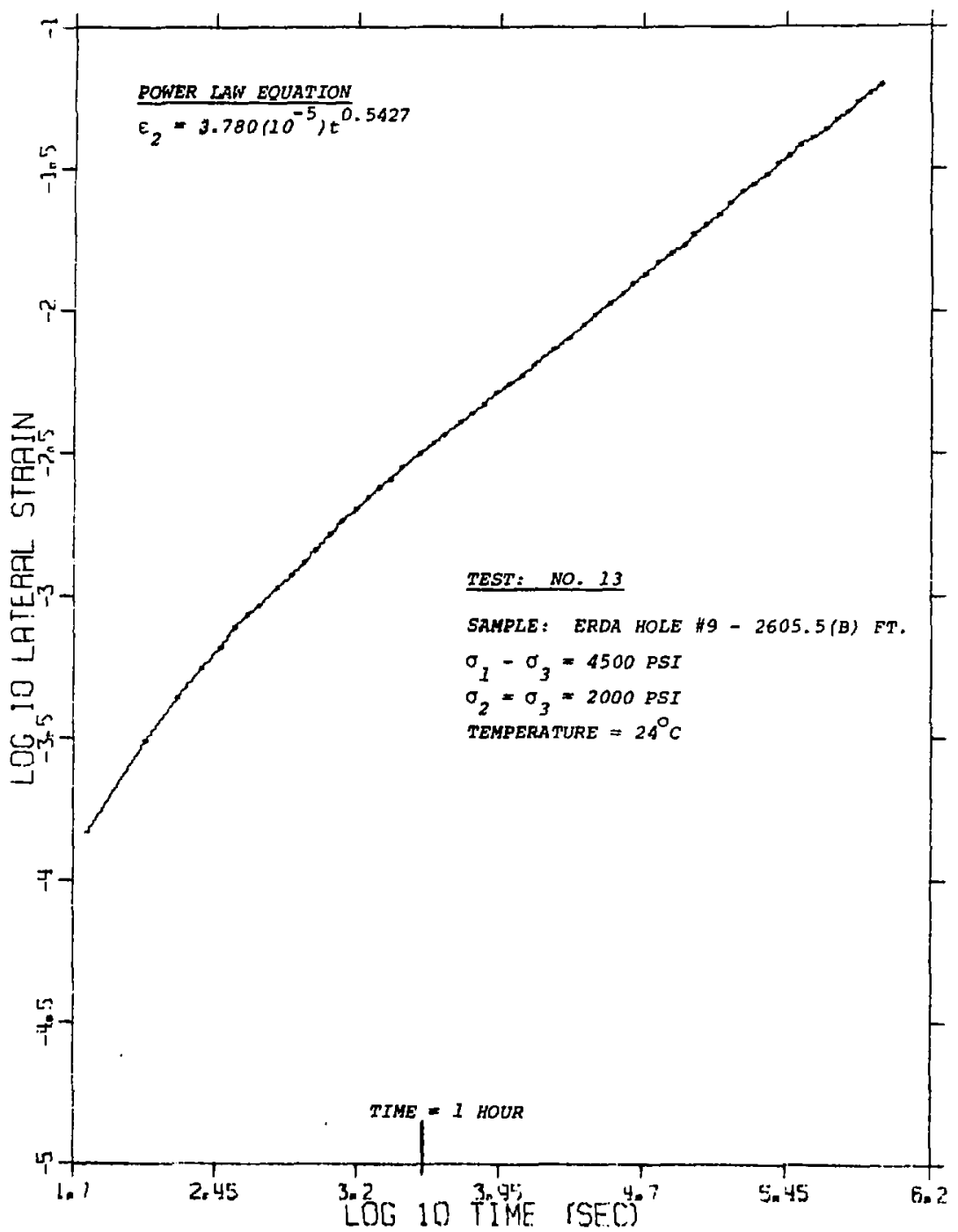

Figure C-19. $\log _{10}$ Lateral Strain as a Function of $\log _{10}$ Time (ace.), Test 13. 


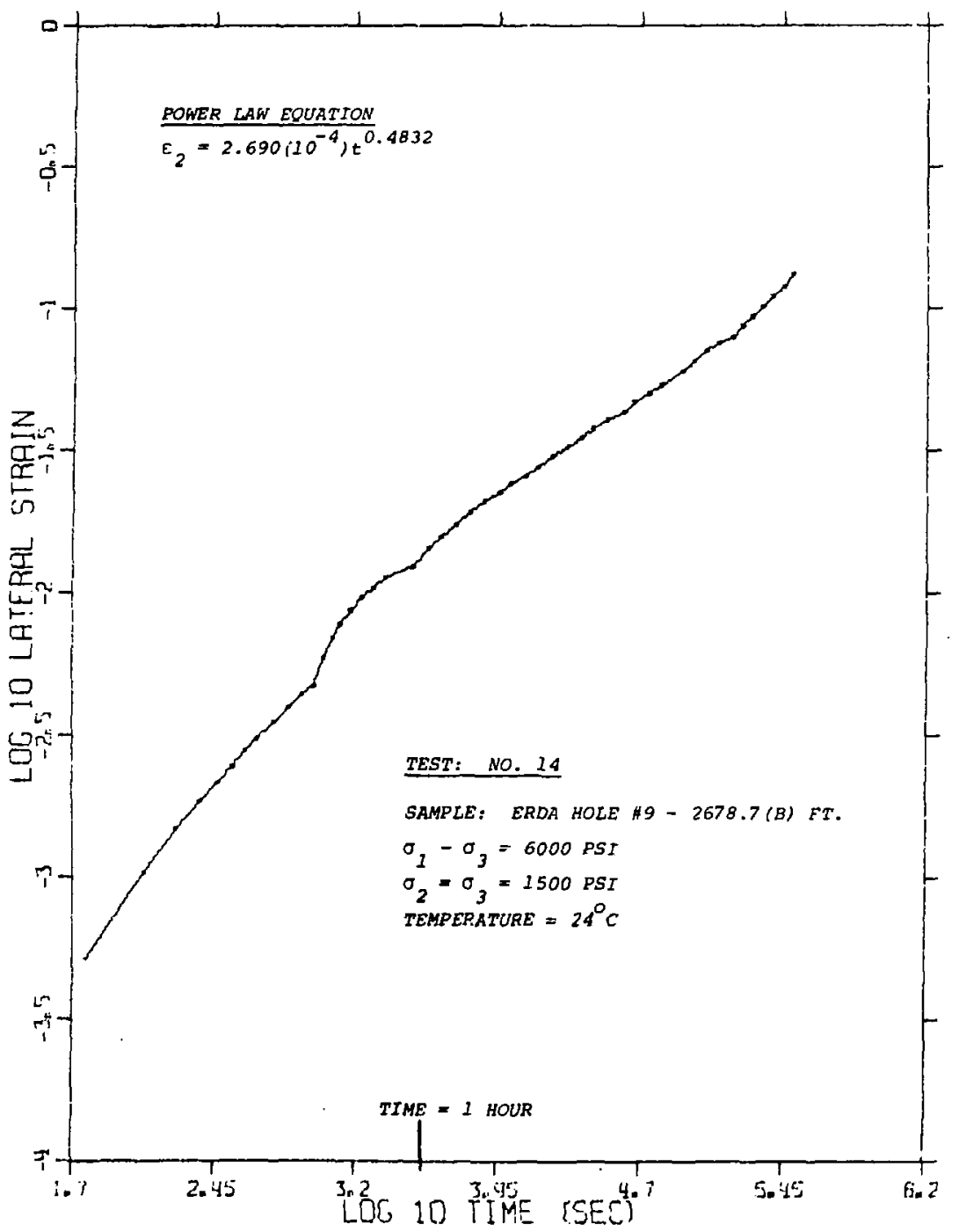

Figure C-20. $\log _{10}$ Lateral strain as a Function of $\log _{10}$ Time (Sec.), rest 14.

120 


\section{APPENDIX D}

SPECTMEN CHARACTERIZATION AND GEOMETRY BEFORE AND AETER TESTING

This special appendix is for the purpose of presenting a physical description of the test specimens as they were set up for testing. In addition, geometrical measurements of length and diameter are given of the specimen before test initiation $\left(L_{0}, D_{0}\right)$ and after test completion $\left(L_{f}, D_{f}\right)$. In some tests the specimens underwent large strains $\left(E_{l}>25 \%\right)$ and the area of the specimen increased such that the piston penetrated the salt, leaving a small circumferential ridge about the ends. In these instances, two measurements of final length are given; $L_{f_{1}}$ is a measurement from piston surface to piston surface while $L_{f_{2}}$ is a measurement from ridge to ridge (approximate). Several measurements of diameter are given for each specimen as barrelling was evident to various degrees in each. Further, cumulative axial strains were calculated $(\Delta L / L)$ using average values and compared to the total axial strain from experimental data. Radial (lateral) strain was similarily calculatec $(\Delta D / D)$. 
TABLE D-I

SPECIMEN CHARACTENIZATION AND GEOMETRIES

\begin{tabular}{|c|c|c|c|c|c|}
\hline $\begin{array}{l}\text { TEST } \\
\text { NO. }\end{array}$ & $\begin{array}{l}\text { SPECIMEN ORIEIN } \\
L_{O}(I N .): D_{O}(I N .)\end{array}$ & SPECIAEN CHARACTERIZATION & Lf & $L_{f_{N}}$ & DIAMETERS (IN.) \\
\hline 1 & $\begin{array}{l}9-2668.5(A) \\
4.096: 1.967\end{array}$ & $\begin{array}{l}\text { very Ig. } x T h, \text { gray impurities, anhydrite idioblasts } \\
\text { epoxy cals in bottom end, few small chips on end }\end{array}$ & 4.070 & -- & $\begin{array}{l}1 / 4=1.97 \\
\text { MTD }=1.98\end{array}$ \\
\hline \multicolumn{3}{|c|}{ 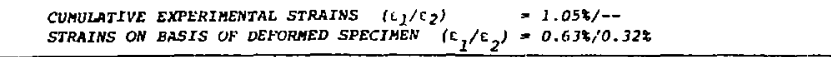 } & & & \\
\hline 2 & $\begin{array}{l}9-2668.5(B) \\
4.055: 1.967\end{array}$ & Excellent edgos, epoxy cap on bottom, small XTL. & \multirow[t]{2}{*}{3.920} & \multirow[t]{2}{*}{--} & \multirow[t]{2}{*}{$\begin{array}{l}1 / 4=2.02 \\
M T D=2.04 \\
1 / 4=2.01\end{array}$} \\
\hline \multicolumn{3}{|c|}{$\begin{array}{lll}\text { CUMULATTVE EXPERTHENT STRAINS }\left(\varepsilon_{1} / \mathrm{c}_{2}\right) & =3.69 \mathrm{t} /-- \\
\text { STRAINS ON BASTS OF DEFORMED SPECTMEN }\left(\varepsilon_{1} / \varepsilon_{2}\right)=3.33 \% / 2.86 \%\end{array}$} & & & \\
\hline 3 & $\begin{array}{l}9-2622.0 \\
4.102: 1.970\end{array}$ & $\begin{array}{l}\text { Excellent adges; very minor cap, small } \\
x T^{L}(0.1 \text { in.) }\end{array}$ & \multirow[t]{2}{*}{3.625} & \multirow[t]{2}{*}{$-\infty$} & \multirow{2}{*}{$\begin{array}{l}1 / 8=2.06 \\
1 / 4=2.20 \\
110=2.24 \\
1 / 4=2.21 \\
1 / 8=2.08\end{array}$} \\
\hline \multicolumn{3}{|c|}{$\begin{array}{l}\text { CUMULATTVE EXPERTMENTAL STRATN }\left(\varepsilon_{1} / \mathrm{c}_{2}\right) \\
\text { STRAINS ON BASTS OF DEFORMED SPECIMEN }\left(\varepsilon_{1} / \mathrm{c}_{2}\right)=11.71 \% /--1.63 \% / 9.548\end{array}$} & & & \\
\hline - & $\begin{array}{l}9-2678.0(A) \\
4.089: 1.973\end{array}$ & $\begin{array}{l}\text { Small chips on one end w/mostiy sm. XTL, other end } \\
1 / 2 \mathrm{sm}, 1 / 2 \text { ig XTL, gray coloration, minor epoxy } \\
\text { cap }\end{array}$ & \multirow[t]{2}{*}{$?$} & \multirow[t]{2}{*}{$?$} & \multirow[t]{2}{*}{$?$} \\
\hline \multicolumn{3}{|c|}{ SPECTMEN FATLED } & & & \\
\hline 5 & $\begin{array}{l}9-2674.5(A) \\
4.003=1.977\end{array}$ & $\begin{array}{l}\text { Very lg. XTL on one end, med, other end, small } \\
\text { xTL along ong edge, minor chips }\end{array}$ & \multirow[t]{2}{*}{3.816} & \multirow[t]{2}{*}{--} & \multirow{2}{*}{$\begin{array}{l}1 / 4=2.998 \\
H I D=2.039 \\
1 / 4=2.022\end{array}$} \\
\hline \multicolumn{3}{|c|}{$\begin{array}{l}\text { CUNULATTVE EXPERTAENTAL STRATNS }\left(\varepsilon_{1} / E_{2}\right) \\
\text { STRAINS ON BASIS OF DEFOFMED SPECIMEN }\left(\varepsilon_{1} / E_{2}\right)=4.90 \% / 2.09 \% \\
=4.67 \% / 2.14 \%\end{array}$} & & & \\
\hline
\end{tabular}


TABLE D-1 (CONT'D)

\begin{tabular}{|c|c|c|c|c|c|}
\hline $\begin{array}{l}\text { TEST } \\
\text { NO. }\end{array}$ & $\begin{array}{l}\text { SPECIMEN JRIGIN } \\
L_{0}(I N .): D_{O}(I N .)\end{array}$ & SPECIMEN CHARACTERTZATION & $\begin{array}{l}L_{f} \\
(I N)\end{array}$ & $\begin{array}{l}I_{f} \\
(I N)\end{array}$ & DIAMETERS (IN.) \\
\hline 6 & $\begin{array}{l}9-2674.5(B) \\
4.083: 1.977\end{array}$ & $\begin{array}{l}\text { most small kTh one end, all small other end, } \\
\text { small throughout, gray coloration }\end{array}$ & \multirow[t]{2}{*}{2.25} & \multirow{2}{*}{$\begin{array}{l}2.41 \\
2.55 \\
2.37\end{array}$} & \multirow{2}{*}{$\begin{array}{l}1 / \theta=2.60 \\
1 / 4=2.67 \\
M I D=2.69 \\
1 / 4=2.64 \\
1 / 8=2.36\end{array}$} \\
\hline \multicolumn{3}{|c|}{$\begin{array}{l}\text { CUMULATIVE EXPERIMENTAL STRAINS }\left(\varepsilon_{1} / \varepsilon_{2}\right) \\
\text { STRAINS ON EASIS OF DEFORRED SPECTMEN }\left(\varepsilon_{1} / \varepsilon_{2}\right)=44.547 / 21.204 \\
\end{array}$} & & & \\
\hline 7 & $\begin{array}{l}9-2679.0(B) \\
4.090: 1.998\end{array}$ & $\begin{array}{l}\text { minor gray impurities, not as much as other } \\
\text { specimens most smill } \mathrm{xTL}(754) \text {, others large (25t) }\end{array}$ & \multirow{2}{*}{$\begin{array}{l}2.31 \\
2.39 \\
2.31\end{array}$} & \multirow{2}{*}{$\begin{array}{l}2.55 \\
2.51 \\
2.50\end{array}$} & \multirow{2}{*}{$\begin{array}{l}1 / E=2.59 \\
1 / 4=2.70 \\
H I D=2.69 \\
1 / 4=2.63 \\
1 / \theta=2.55\end{array}$} \\
\hline \multicolumn{3}{|c|}{ 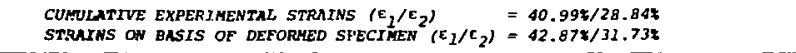 } & & & \\
\hline 8 & $\begin{array}{l}9-2605.0(\theta) \\
1.042: 1.99\end{array}$ & $\begin{array}{l}\text { Most small XTL one end } w / 1 g . \text { XTL in center, } \\
\text { otherwise } \operatorname{small} \text { XTL, gray impurities throughout }\end{array}$ & \multirow[t]{2}{*}{3.771} & \multirow[t]{2}{*}{-} & \multirow{2}{*}{$\begin{array}{l}1 / 8=2.06 \\
1 / 4=2.09 \\
M I D=2.08 \\
1 / 4=2.06 \\
1 / 8=2.04\end{array}$} \\
\hline \multicolumn{3}{|c|}{ 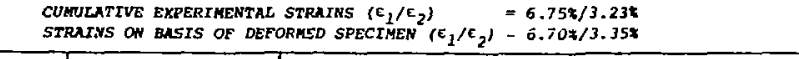 } & & & \\
\hline 9 & $\begin{array}{l}9-2678.0(B) \\
4.096: 1.967\end{array}$ & one small chip on top; large XTLs & \multirow[t]{2}{*}{$?$} & \multirow[t]{2}{*}{-} & \multirow[t]{2}{*}{$?$} \\
\hline \multicolumn{3}{|c|}{ SPECINEN FATLED } & & & \\
\hline 10 & $\begin{array}{l}9-2606.0(B) \\
4.026: 1.998\end{array}$ & Snall XTL, minor impurities & \multirow[t]{2}{*}{3.87} & \multirow[t]{2}{*}{-} & \multirow{2}{*}{$\begin{array}{l}1 / 4=2.03 \\
M I D=2.04 \\
1 / 4=2.0 .7\end{array}$} \\
\hline \multicolumn{3}{|c|}{ 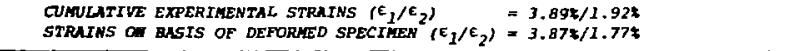 } & & & \\
\hline
\end{tabular}


TAOLE D-1 (COMT'D)

\begin{tabular}{|c|c|c|c|c|c|}
\hline $\begin{array}{l}\text { TEST } \\
\text { NO. }\end{array}$ & $\begin{array}{l}\text { SPECTREN ORTGTN } \\
I_{0}(T N .): D_{0}(T N .)\end{array}$ & SPECTHEN CHARACTERTZATION & $\begin{array}{l}L_{f_{1}} \\
(I N)\end{array}$ & $\begin{aligned} L_{f 2} \\
(I N)\end{aligned}$ & DLAMETERS (IN-) \\
\hline 11 & $\begin{array}{l}9-2679.0(A) \\
4.032: 1.998\end{array}$ & $\begin{array}{l}\text { Most large XTL, minor impurities upper end, } \\
\text { arrow points to upper end }\end{array}$ & \multirow{2}{*}{$\begin{array}{l}3.301 \\
3.296 \\
3.290\end{array}$} & $\begin{array}{l}4.10 \\
3.01\end{array}$ & \multirow{2}{*}{$\begin{array}{l}1 / 8=2.31 \\
1 / 4=2.34 \\
H 1 D=2.37 \\
1 / 4=2.34 \\
1 / 8=2.30\end{array}$} \\
\hline \multicolumn{3}{|c|}{$\begin{array}{ll}\text { CUNULATIVE EXPERTHENTAL STRAINS }\left(\mathrm{E}_{1} / \varepsilon_{2}\right) & =15.50 \% /- \\
\text { STRAINS ON BASIS OF DEFORMED SPECIMEN }\left(\mathrm{\varepsilon}_{1} / \varepsilon_{2}\right)=18.26 \% / 16.727\end{array}$} & & EDGES & \\
\hline 12 & $\begin{array}{l}9-2678.3(0) \\
4.061: 1.995\end{array}$ & Smal' to medium XTL, gray coloration & \multirow[t]{2}{*}{2.991} & $\begin{array}{l}3.11 \\
3.06\end{array}$ & \multirow{2}{*}{$\begin{array}{l}1 / 8=2.25 \\
1 / 4=2.33 \\
H I D=2.42 \\
1 / 4=2.30 \\
1 / 8=2.26\end{array}$} \\
\hline \multicolumn{3}{|c|}{$\begin{array}{ll}\text { CUNULATIVE EXPERIMENTAL STRAINS }\left(E_{1} / C_{2}\right) & =27.70 \% / 14.72 \% \\
\text { STRAINS ON BASIS OF DEFORMED SPECTMEN }\left(\varepsilon_{1} / \varepsilon_{2}\right) & =26.35 \% / 15.89 \%\end{array}$} & & & \\
\hline 13 & $\begin{array}{l}9-2605.5(B) \\
4.077 .1 .998 \\
\end{array}$ & Small $x T L$, minor impurities & \multirow[t]{2}{*}{3.49} & $\begin{array}{l}3.53 \\
3.52\end{array}$ & \multirow{2}{*}{$\begin{array}{l}1 / 8=2.13 \\
1,4=2.19 \\
H I D=2.18 \\
1 / 4=2.18 \\
1 / B=2.11\end{array}$} \\
\hline \multicolumn{3}{|c|}{$\begin{array}{lll}\text { CUMULATIVT EAPERIMENTAL STRAINS }\left(\varepsilon_{1} / \mathrm{C}_{2}\right) & =12.96 \% / 6.81 \% \\
\text { STRAINS ON DASIS OF DEFORMED SPECIMEN }\left(\varepsilon_{1} / c_{2}\right)=14.40 \% / B_{.} .01 \%\end{array}$} & & & \\
\hline 14 & $\begin{array}{l}9-2678.7(B) \\
4.018: 1.997\end{array}$ & $\begin{array}{l}\text { Small to medium XTL, gray impurities } \\
\text { diagonally oriented }\end{array}$ & \multirow[t]{2}{*}{2.90} & \multirow{2}{*}{$\begin{array}{l}3.08 \\
3.01 \\
3.10\end{array}$} & \multirow{2}{*}{$\begin{array}{l}1 / 8=2.29 \\
1 / 4=2.38 \\
M I D=2.41 \\
I / 4=2.50 \\
1 / 8=2.31\end{array}$} \\
\hline \multicolumn{3}{|c|}{ 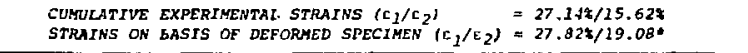 } & & & \\
\hline
\end{tabular}

